\title{
Exploring molecular complexity with ALMA (EMoCA): Alkanethiols and alkanols in Sagittarius B2(N2)
}

\author{
Holger S. P. Müller ${ }^{1}$, Arnaud Belloche ${ }^{2}$, Li-Hong Xu (徐丽红) $)^{3}$, Ronald M. Lees ${ }^{3}$, Robin T. Garrod ${ }^{4}$, Adam Walters ${ }^{5,6}$, \\ Jennifer van Wijngaarden ${ }^{7}$, Frank Lewen ${ }^{1}$, Stephan Schlemmer ${ }^{1}$, and Karl M. Menten ${ }^{2}$
}

1 I. Physikalisches Institut, Universität zu Köln, Zülpicher Str. 77, 50937 Köln, Germany e-mail: hspm@ph1.uni-koeln.de

2 Max-Planck-Institut für Radioastronomie, Auf dem Hügel 69, 53121 Bonn, Germany

${ }^{3}$ Centre for Laser, Atomic and Molecular Sciences (CLAMS), Department of Physics, University of New Brunswick, Saint John, NB E2L 4L5, Canada

${ }^{4}$ Departments of Chemistry and Astronomy, University of Virginia, Charlottesville, VA 22904, USA

5 Université de Toulouse, UPS-OMP, IRAP, Toulouse, France

${ }^{6}$ CNRS, IRAP, 9 Av. colonel Roche, BP 44346, 31028 Toulouse Cedex 4, France

7 Department of Chemistry, University of Manitoba, Winnipeg, MB R3T 2N2, Canada

Received 29 September 2015 / Accepted 24 November 2015

\begin{abstract}
Context. Over the past five decades, radio astronomy has shown that molecular complexity is a natural outcome of interstellar chemistry, in particular in star forming regions. However, the pathways that lead to the formation of complex molecules are not completely understood and the depth of chemical complexity has not been entirely revealed. In addition, the sulfur chemistry in the dense interstellar medium is not well understood.

Aims. We want to know the relative abundances of alkanethiols and alkanols in the Galactic center source Sagittarius B2(N2), the northern hot molecular core in Sgr B2(N), whose relatively small line widths are favorable for studying the molecular complexity in space.

Methods. We investigated spectroscopic parameter sets that were able to reproduce published laboratory rotational spectra of ethanethiol and studied effects that modify intensities in the predicted rotational spectrum of ethanol. We used the Atacama Large Millimeter Array (ALMA) in its Cycles 0 and 1 for a spectral line survey of Sagittarius B2(N) between 84 and 114.4 GHz. These data were analyzed by assuming local thermodynamic equilibrium (LTE) for each molecule. Our observations are supplemented by astrochemical modeling; a new network is used that includes reaction pathways for alkanethiols for the first time.

Results. We detected methanol and ethanol in their parent ${ }^{12} \mathrm{C}$ species and their isotopologs with one ${ }^{12} \mathrm{C}$ atom substituted by ${ }^{13} \mathrm{C}$; the latter were detected for the first time unambiguously in the case of ethanol. The ${ }^{12} \mathrm{C} /{ }^{13} \mathrm{C}$ ratio is $\sim 25$ for both molecules. In addition, we identified $\mathrm{CH}_{3}^{18} \mathrm{OH}$ with a ${ }^{16} \mathrm{O} /{ }^{18} \mathrm{O}$ ratio of $\sim 180$ and a ${ }^{13} \mathrm{CH}_{3} \mathrm{OH} / \mathrm{CH}_{3}^{18} \mathrm{OH}$ ratio of $\sim 7.3$. Upper limits were derived for the next larger alkanols normal- and iso-propanol. We observed methanethiol, $\mathrm{CH}_{3} \mathrm{SH}$, also known as methyl mercaptan, including torsionally excited transitions for the first time. We also identified transitions of ethanethiol (or ethyl mercaptan), though not enough to claim a secure detection in this source. The ratios $\mathrm{CH}_{3} \mathrm{SH}$ to $\mathrm{C}_{2} \mathrm{H}_{5} \mathrm{SH}$ and $\mathrm{C}_{2} \mathrm{H}_{5} \mathrm{OH}$ to $\mathrm{C}_{2} \mathrm{H}_{5} \mathrm{SH}$ are $\gtrsim 21$ and $\gtrsim 125$, respectively. In the process of our study, we noted severe discrepancies in the intensities of observed and predicted ethanol transitions and propose a change in the relative signs of the dipole moment components. In addition, we determined alternative sets of spectroscopic parameters for ethanethiol. The astrochemical models indicate that substantial quantities of both $\mathrm{CH}_{3} \mathrm{SH}$ and $\mathrm{C}_{2} \mathrm{H}_{5} \mathrm{SH}$ may be produced on the surfaces of dust grains, to be later released into the gas phase. The modeled ratio $\mathrm{CH}_{3} \mathrm{SH} / \mathrm{C}_{2} \mathrm{H}_{5} \mathrm{SH}=3.1$ is lower than the observed value of $\gtrsim 21$; the model value appears to be affected most by the underprediction of $\mathrm{CH}_{3} \mathrm{SH}$ relative to $\mathrm{CH}_{3} \mathrm{OH}$ and $\mathrm{C}_{2} \mathrm{H}_{5} \mathrm{OH}$, as judged by a very high $\mathrm{CH}_{3} \mathrm{OH} / \mathrm{CH}_{3} \mathrm{SH}$ ratio.

Conclusions. The column density ratios involving methanol, ethanol, and methanethiol in Sgr B2(N2) are similar to values reported for Orion KL, but those involving ethanethiol are significantly different and suggest that the detection of ethanethiol reported toward Orion KL is uncertain. Our chemical model presently does not permit the prediction of sufficiently accurate column densities of alkanethiols or their ratios among alkanethiols and alkanols. Therefore, additional observational results are required to establish the level of $\mathrm{C}_{2} \mathrm{H}_{5} \mathrm{SH}$ in the dense and warm interstellar medium with certainty.
\end{abstract}

Key words. astrochemistry - line: identification - molecular data - radio lines: ISM - ISM: individual objects: Sagittarius B2(N) ISM: molecules

\section{Introduction}

The degree of chemical complexity observed toward starforming regions known as "hot cores" is greater than that found thus far in any other portion of the interstellar medium (ISM), encompassing a range of organic molecules of up to 12 atoms (Belloche et al. 2014). The significance of these detections may go beyond the immediate chemistry of hot cores themselves; the apparent similarity between the abundances of complex interstellar molecules and the chemical composition of comets 
suggests that interstellar material may have been incorporated directly into cometary and/or planetary solids during the planet formation process. It is therefore critical to understand the depth and breadth of chemical complexity that may develop in the early stages of star formation, and which may influence the chemistry of later planetary systems and perhaps the ultimate emergence of life.

Sagittarius (Sgr for short) B2 is an especially well-suited source for studying saturated or nearly saturated organic molecules, and most of the larger ones have been detected toward this source for the first time (Belloche et al. 2013). Sgr B2(N) and Sgr B2(M) are the two main sites of star formation in the Sgr B2 molecular cloud complex, one of the most massive star-forming regions in our Galaxy located close to the Galactic center. Sgr B2(N) contains two dense, compact hot cores separated by about 5" (Belloche et al. 2008; Qin et al. 2011), the more prominent one is the so-called Large Molecule Heimat Sgr B2(N-LMH), also called Sgr B2(N)-SMA1 (Qin et al. 2011) or P1 (Belloche et al. 2008); the other is called Sgr B2(N)-SMA2 (Qin et al. 2011) or P2 (Belloche et al. 2008) and is to the north of the first. We propose that Sgr B2(N1) and Sgr B2(N2) be adopted because they are short and unambiguous names. Single-dish observations of Sgr B2(N) usually cover both sources, but differences in the local standard of rest (lsr) velocities provide the means to distinguish between the two sources.

In order to study the molecular complexity in Sgr B2, we used the IRAM $30 \mathrm{~m}$ telescope to carry out molecular line surveys toward Sgr B2(N) and Sgr B2(M) at $3 \mathrm{~mm}$ wavelengths with additional observations at 2 and $1.3 \mathrm{~mm}$ (Belloche et al. 2013). Even though each frequency setup was observed for a modest amount of time, line confusion was reached for a considerable part of the surveys, in particular toward Sgr B2(N). Nevertheless, in this study we detected aminoacetonitrile, a possible precursor of glycine (the simplest amino acid), for the first time in space (Belloche et al. 2008), as well as ethyl formate and $n$-propyl cyanide (Belloche et al. 2009), the ${ }^{13} \mathrm{C}$ isotopologs of vinyl cyanide (Müller et al. 2008), and several other minor isotopic species and transitions in excited vibrational states (Belloche et al. 2013).

Other recent investigations into the molecular complexity of Sgr B2(N) include a line survey with the HIFI high-resolution spectrometer on board Herschel covering its entire frequency region (most of $\sim 500$ to $\sim 1900 \mathrm{GHz}$ ) (Neill et al. 2014) or the PRIMOS Legacy Project (up to about $50 \mathrm{GHz}$ ) employing the $100 \mathrm{~m}$ GBT dish, which led to the detection of ethanimine (Loomis et al. 2013) and E-cyanomethanimine (Zaleski et al. 2013), among others. Among the less recent studies of Sgr B2, we note the $1.3 \mathrm{~mm}$ line survey of Nummelin et al. (1998, 2000) who studied three positions (M, N, and NW) using the SEST $15 \mathrm{~m}$ dish.

The Orion Molecular Cloud, and in particular the Kleinmann-Low Nebula (Orion KL), have also been studied extensively in the context of molecular complexity (Crockett et al. 2014). An IRAM $30 \mathrm{~m}$ molecular line survey led to the detection of several molecules including methyl acetate (Tercero et al. 2013) (an isomer of ethyl formate), and numerous minor isotopic species, such as methyl-deuterated methyl formate, $\mathrm{HCO}(\mathrm{O}) \mathrm{CH}_{2} \mathrm{D}$ (Coudert et al. 2013). The detection of methyl formate in its second excited torsional state was reported through different observations (Takano et al. 2012). In recent years, considerable levels of molecular complexity have also been investigated in other star-forming regions. Ethanediol, for example, which is also known as ethylene glycol, is a molecule with ten atoms. It was detected in the hot corinos associated with the lower mass Class 0 protostars NGC 1333-IRAS2A (Maury et al. 2014) and, tentatively, IRAS 16293-2422B (Jørgensen et al. 2012) after the first detection toward Sgr B2(N) (Hollis et al. 2002).

Following up on our observations with the IRAM 30 m telescope, we used the Atacama Large Millimeter Array (ALMA) in its Cycle 0 for a spectral line survey of Sagittarius B2(N) between 84 and $111 \mathrm{GHz}$ with the aim of Exploring Molecular Complexity with ALMA (EMoCA). An additional setup in Cycle 1 extended the frequency range to $114.4 \mathrm{GHz}$. We expected a reduction in the line confusion through the spatial resolution of Sgr B2(N1) and Sgr B2(N2). In fact, we observed reduced line confusion toward the weaker source Sgr B2(N2) caused not only by the spatial separation of the two sources, but also by narrower lines. As a first result, we were able to detect the first branched alkyl molecule in space, iso-propyl cyanide and to determine a $1: 2.5$ ratio relative to the more abundant $n$-propyl cyanide in Sgr B2(N2) (Belloche et al. 2014). The line confusion is high in Sgr B2(N1), therefore, we focus our initial analyses on Sgr B2(N2). In the present work, we report on alkanols and alkanethiols in this source.

Methanol, $\mathrm{CH}_{3} \mathrm{OH}$, is the lightest alkanol and one of the most abundant organic molecules in the ISM. It was among the first molecules to be detected in space, toward Sgr A and Sgr B2, by means of radio astronomy (Ball et al. 1970). In fact, ${ }^{13} \mathrm{CH}_{3} \mathrm{OH}$ and $\mathrm{CH}_{3} \mathrm{OD}$ were detected in Sgr B2 shortly thereafter (Gottlieb et al. 1979). We note, however, that the identification of $\mathrm{CH}_{3} \mathrm{OD}$ in that work has been questioned recently (Belloche et al. 2016). A few years later, Gardner et al. (1989) reported on the detection of $\mathrm{CH}_{3}^{18} \mathrm{OH}$ in $\mathrm{Sgr}$ B2. The great diagnostic value of methanol lines has been pointed out several times (Leurini et al. 2004, 2007; Müller et al. 2004; Wang et al. 2011). Rotational lines of ${ }^{13} \mathrm{CH}_{3} \mathrm{OH}$ have been used frequently to derive proper column densities because those of the main isotopic species are often saturated in dense molecular clouds (Belloche et al. 2013; Neill et al. 2013).

Ethanol, $\mathrm{C}_{2} \mathrm{H}_{5} \mathrm{OH}$, the next heaviest alkanol, was also among the first molecules to be detected in space employing radio astronomy (Zuckerman et al. 1975). The detection was also made toward Sgr B2. Initially, it was observed only via its anti (or trans) low-energy conformer, the higher lying gauche conformer was first detected in Orion KL several years later (Pearson et al. 1997). Ethanol was not only detected in several hot cores, but also in kinetically moderately warm Galactic center clouds (Requena-Torres et al. 2006). In addition, there is evidence for the molecule in the molecular clouds surrounding the Class 0 protostars IRAS 16293-2422A and B (Bisschop et al. 2008). Ethanol with one ${ }^{13} \mathrm{C}$ has not yet been detected with certainty, but we reported on a tentative detection in the course of the $30 \mathrm{~m}$ line survey of Sgr B2(N) (Belloche et al. 2013).

In the case of propanol, $\mathrm{C}_{3} \mathrm{H}_{7} \mathrm{OH}$, there are two isomers, the straight chain normal- or $n$-propanol and the branched iso- or $i$-propanol. We are aware of two reports attempting to detect $n$-propanol in space (Requena-Torres et al. 2008; Tercero et al. 2015). There appears to be no published report on the search for $i$-propanol in space.

Replacing an $\mathrm{O}$ atom in an alkanol with an $\mathrm{S}$ atom yields an alkanethiol. The simplest one is methanethiol, $\mathrm{CH}_{3} \mathrm{SH}$, also known as methyl mercaptan. It was among the early molecules detected in space by radio astronomical means, first tentatively (Turner 1977) and confirmed subsequently (Linke et al. 1979), again toward Sgr B2. It was also observed toward the hot core G327.3-0.6 (Gibb et al. 2000), the cold core B1 (Cernicharo et al. 2012), and the Orion KL hot core (Kolesniková et al. 2014). The 
last study also reported the detection of ethanethiol, $\mathrm{C}_{2} \mathrm{H}_{5} \mathrm{SH}$, also known as ethyl mercaptan. Even though many spectral features were ascribed to the molecule, most of them are partially or completely blended with emission from other species. In addition, the column density ratio of ethanethiol with respect to methanethiol or ethanol appears somewhat higher than the column density ratios of the latter two molecules with respect to methanol. The puzzlingly high apparent abundance of $\mathrm{C}_{2} \mathrm{H}_{5} \mathrm{SH}$ in Orion KL motivates probing the abundances of alkanols and alkanethiols in other hot cores, in particular Sgr B2(N), in order to understand better the interstellar chemistry of these two families of organic molecules.

\section{Laboratory spectroscopy}

In this section, we present background information on the spectroscopy of the molecules in the present study. One general aspect concerns the partition function of organic molecules and the related abundances. These molecules often have low-lying vibrations, such as $\mathrm{OH}$-, $\mathrm{SH}$-, or $\mathrm{CH}_{3}$-torsions, which are not always considered in the derivation of the partition function. We comment on necessary corrections of the partition function and, equivalently, the column densities under local thermodynamic equilibrium (LTE). Similar corrections may be required if not all of the thermally populated conformations were considered in the derivation of the partition function.

The rotational spectrum of a saturated or nearly saturated organic molecule may differ considerably from that of a simple asymmetric top rotor because the torsion or inversion of functional groups such as $\mathrm{CH}_{3}, \mathrm{NH}_{2}, \mathrm{OH}$, or $\mathrm{SH}$ may be hindered only slightly. These large amplitude motions may lead to additional splitting in the rotational transitions, which sometimes can be resolved in radio astronomical spectra.

Pickett (1972) proposed a reduced axis system (RAS) for the treatment of inversion problems, i.e., tunneling between two equivalent minima. Its main off-diagonal term of $a$-symmetry is $F_{b c}\left\{J_{b}, J_{c}\right\}$ with $\left\{J_{b}, J_{c}\right\}=J_{b} J_{c}+J_{c} J_{b}$. Distortion corrections, such as $\left\{\left\{J_{b}, J_{c}\right\}, F_{b c, K} J_{a}^{2}+F_{b c, J} J^{2}+F_{2 b c}\left(J_{+}^{2}+J_{-}^{2}\right)+\ldots\right\}$ with $J_{ \pm}=$ $J_{b} \pm i J_{c}$, may also be required. Terms of $b$ - and $c$-symmetry are defined equivalently. The symmetry of the allowed terms in the Hamiltonian depends on the symmetry of the inversion motion. The designation of the parameter as $F_{b c}$ is fairly common, but other designations also exist (e.g., $D_{b c}$ ). Parameters such as $F_{2 b c}$ may also be called $F_{b c}^{ \pm}$.

Reduced axis system Hamiltonians were employed successfully for a variety of molecules of interest for astrochemistry, including $\mathrm{NH}_{2} \mathrm{D}$ (Cohen \& Pickett 1982), vinylamine (McNaughton \& Evans 1999), aGg'-ethanediol (Christen \& Müller 2003), $\mathrm{NHD}_{2}$ (Endres et al. 2006), $\mathrm{H}_{2} \mathrm{DO}^{+}$(Müller et al. 2010), phenol (Kolesniková et al. 2013), and 1,3-propanediol (Smirnov et al. 2013).

A RAS Hamiltonian still appears to be well suited to describe the tunneling between two equivalent conformations if a third minimum is sufficiently higher in energy. This third minimum usually has a dihedral angle of $\sim 0^{\circ}(s y n)$ or $\sim 180^{\circ}$ (anti); the two equivalent conformations have dihedral angles of about $\pm 120^{\circ}$ or of about $\pm 60^{\circ}$, respectively. In either case, the conformations are frequently called gauche. Examples are peroxynitric acid (Suenram et al. 1986a), fluoromethanol (Suenram et al. 1986b), and propargyl alcohol (Pearson \& Drouin 2005). If a third minimum is closer in energy to the gauche states, it may be necessary to include parameters of odd order into the Hamiltonian to describe the tunneling between the gauche states. The lower order parameters of $a$-symmetry are $\left\{i J_{a}, G_{a}+G_{a, K} J_{a}^{2}+G_{a, J} J^{2}+G_{2}\left(J_{+}^{2}+\right.\right.$
$\left.\left.J_{-}^{2}\right)+\ldots\right\}$, those of $b$ - and $c$-symmetry are defined equivalently. This approach has been used, for example, in the treatment of the rotation-tunneling spectrum of ethanol (Pearson et al. 1996, 2008).

In the case of tunneling between two equivalent conformations if a third minimum is sufficiently higher in energy, the RAS interaction Hamiltonian with even order parameters, such as $F_{a b}$, may be replaced by an interaction Hamiltonian with odd order parameters, such as $G_{c}$. Pearson \& Drouin (2005) have shown that equivalent fits can be obtained with only even or with only odd order interaction parameters in the case of propargyl alcohol.

We note that the sign of the first off-diagonal interaction parameter of a given symmetry and between specific conformations or vibrational states is not determinable in a fit of field-free rest frequencies. This parameter is usually the one of lowest order, i.e., $G_{i}$ or $F_{j k}$. However, the sign of any additional interaction parameter of the same symmetry and between those specific conformations or vibrational states are determinable with respect to that of the first parameter. Moreover, in the absence of offdiagonal interaction parameters, the signs of the dipole moment components are not determinable and do not matter. However, in the presence of off-diagonal interaction parameters and with their signs fixed, the relative signs of the dipole moment components may matter; in fact, it is the sign of the dipole moment component times the sign of the overlap integral that is determined. In that case, they may be determinable by Stark spectroscopy (Cohen \& Pickett 1982; Suenram et al. 1986a,b). The relative signs of the dipole moment components may also lead to changes in the intensities of some transitions, and conversely, relative intensity measurements may lead to the determination of the relative signs of the dipole moment components (Christen \& Müller 2003; Endres et al. 2006; Müller et al. 2010).

The treatment of torsional large amplitude motions (e.g., of $\mathrm{CH}_{3}$ groups) often requires more specialized Hamiltonian models (see, e.g., Herbst et al. 1984 or Kleiner 2010 for a review) because the regular $K$ energy level structure of an asymmetric top rotor could be modified significantly by torsional contributions that oscillate as a function of $K$. The amplitude of this oscillation increases rapidly with excitation to torsional states of successively higher energy relative to the torsional barrier. The frequency of the $K$-variation depends on the ratio of the moment of inertia of the rotating top to that of the whole molecule, hence torsional contributions to the energies oscillate faster with $K$ for heavy tops and light frames.

\subsection{Methanol, $\mathrm{CH}_{3} \mathrm{OH}$}

The torsional energy for methanol varies rapidly with $K$ because the moment of inertia of the $\mathrm{CH}_{3}$ group dominates that of the light $\mathrm{OH}$ frame. In addition, the intermediate-size barrier to rotation of the $\mathrm{CH}_{3}$ group leads to torsional contributions to the $K$ energy level structure in the ground vibrational state that are of similar size to the $K$ level splitting at low $K$. As a consequence, the origins of the $b$-type rotational transitions (with $\Delta K=1$ ) differ considerably from those expected for an asymmetric top rotor. In contrast, the patterns of the $a$-type rotational transitions (with $\Delta K=0$ ) resemble those of an asymmetric top in the absence of perturbations; this applies even to excited torsional states. The first excited torsional state straddles the barrier to internal rotation, and even higher torsional states display a $K$-level structure that increasingly approaches that of a free internal rotor. 
We have used rotational transitions of ${ }^{13} \mathrm{CH}_{3} \mathrm{OH}$ initially to infer the column density of methanol because of frequent saturation of lines pertaining to the main isotopic species. The spectroscopic line data were taken from the CDMS catalog ${ }^{1}$ (Müller et al. 2001, 2005). The first version is based on Xu et al. (1996) and Xu \& Lovas (1997) with experimental transition frequencies in the range of our ALMA data from Kuriyama et al. (1986) and from the privately communicated methanol atlas of the Toyama University (Tsunekawa et al. 1995). An update of the CDMS entry is in preparation. It is based on extensive measurements taken in Cologne that extend up to $\sim 1500 \mathrm{GHz}$ and include data in the range of our survey ( $\mathrm{Xu}$ et al. 2014). The magnitudes of the dipole moment components employed in the calculation are $\left|\mu_{a}\right|=0.899 \mathrm{D}$ and $\left|\mu_{b}\right|=1.44 \mathrm{D}$ from initial measurements on the main isotopic species (Ivash \& Dennison 1953). Sastry et al. (1994) determined slightly different dipole moment components of ${ }^{13} \mathrm{CH}_{3} \mathrm{OH}$. The vibrational contributions to the partition function at $160 \mathrm{~K}$ are essentially converged by inclusion of $v_{\mathrm{t}}=2$, a correction of the column density because of the population of vibrational states is not required.

We used the CDMS entry to evaluate the column density of $\mathrm{CH}_{3}^{18} \mathrm{OH}$. The entry is based on Fisher et al. (2007) with extensive far-infrared data from that work. Most of the rest frequencies determined with microwave accuracy are from Hoshino et al. (1996) and from Ikeda et al. (1998). The dipole moment components are slightly different from those of the main species and were taken from Sastry et al. (1996). As for ${ }^{13} \mathrm{CH}_{3} \mathrm{OH}$, vibrational corrections to the partition function at $160 \mathrm{~K}$ are not needed.

We used the JPL catalog ${ }^{2}$ (Pickett et al. 1998) entry to estimate the $\mathrm{CH}_{3} \mathrm{OH}$ column density because we expect to observe highly rotationally or torsionally excited $\mathrm{CH}_{3} \mathrm{OH}$ transitions with line widths much wider than in dark clouds. The entry is based on the extensive study of Xu et al. (2008) which extends to $v_{\mathrm{t}}=2$ and high $J$ and $K$ quantum numbers. Rest frequencies in the range of our study were taken mainly from the methanol atlas of the Toyama University ${ }^{3}$ (Tsunekawa et al. 1995) with additional data from Lees \& Baker (1968), Sastry et al. (1984), Müller et al. (2004), Xu et al. (2008). An older entry based on Xu \& Lovas (1997) is available in the CDMS. Even though it covers only $v_{\mathrm{t}}=0$ and 1 and has weaknesses for transitions with higher $J$ and $K$ values, it is frequently more accurate for lower energy transitions that may be important for dark cloud or maser observations, which require high frequency accuracy. This is achieved in part by merging corresponding laboratory data (Müller et al. 2004). The dipole moments were taken from Ivash \& Dennison (1953). Very recently, determinations of the dipole moment components were reported by Mukhopadhyay \& Sastry (2015) with very similar values. The small change in the $a$-dipole moment component upon torsional excitation probably has negligible effects for the strongest $a$-type transitions even in a perfect fit of astronomical data. There may be non-negligible effects in transitions in which contributions of the $a$ - and $b$-dipole moment components almost cancel out. Vibrational corrections to the partition function at $160 \mathrm{~K}$ are again not needed.

We suspected that transitions pertaining to $v_{\mathrm{t}}=3$ may be observable in our data. We took $J=2-1 a$-type transition frequencies from Tsunekawa et al. (1995), the lower state energies from Moruzzi et al. (1995), and estimated the line strengths from

\footnotetext{
1 http://www . astro.uni-koeln.de/cdms/catalog; http://cdms.ph1.uni-koeln.de/cdms/portal/

2 http://spec.jpl.nasa.gov/ftp/pub/catalog/catdir.html

3 http://wwW.sci.u-toyama.ac.jp/phys/4ken/atlas/
}

lower torsional states (Xu et al. 2008). It is worthwhile mentioning that substantial progress has been made recently in analyzing $v_{\mathrm{t}}=3$ and higher vibrational states of methanol (Pearson et al. 2009, 2015).

\subsection{Methanethiol, $\mathrm{CH}_{3} \mathrm{SH}$}

Replacing the $\mathrm{O}$ atom in methanol with an $\mathrm{S}$ atom yields methanethiol, reducing the moment of inertia ratio between the $\mathrm{CH}_{3}$ group and the $\mathrm{SH}$ frame compared to the $\mathrm{OH}$ frame in methanol. Overall, the torsional splitting in $\mathrm{CH}_{3} \mathrm{SH}$ is smaller than that in $\mathrm{CH}_{3} \mathrm{OH}$. The splittings in the first excited torsional state of $\mathrm{CH}_{3} \mathrm{SH}$ are similar to the ones in the ground vibrational state of $\mathrm{CH}_{3} \mathrm{OH}$.

Information on rest frequencies were taken from the CDMS. The entry is based on $\mathrm{Xu}$ et al. (2012). Extensive terahertz and far-IR data were combined with previous data, which include data in the range of our survey from Lees \& Mohammadi (1980) with additional data mainly from Sastry et al. (1986) and Bettens et al. (1999). Dipole moment components of $\left|\mu_{a}\right|=1.312 \mathrm{D}$ and $\left|\mu_{b}\right|=0.758 \mathrm{D}$ were cited by Tsunekawa et al. (1989). The vibrational contributions to the partition function are quite well accounted for at $180 \mathrm{~K}$, so no correction to the column density needs to be made.

\subsection{Ethanol, $\mathrm{C}_{2} \mathrm{H}_{5} \mathrm{OH}$}

There are two large amplitude motions in ethanol, the internal rotation of the methyl group, whose rather small splitting is only rarely resolved in the laboratory and is frequently neglected, and the internal rotation of the $\mathrm{OH}$ group, which causes more prominent splitting. The anti conformer (also known as trans) with a dihedral HOCC angle of $180^{\circ}$ is lowest in energy. The doubly degenerate gauche conformer with dihedral angles of about $\pm 60^{\circ}$ is considerably higher in energy. Tunneling between the two equivalent gauche conformers causes them to split into the symmetric gauche ${ }^{+}$and the antisymmetric gauche $e^{-}$states. The former is $56.82 \mathrm{~K}$ higher than the anti conformer and the latter 61.53 K (Pearson et al. 2008). Torsion-rotation interaction between the gauche conformers occurs even at low quantum numbers (Pearson et al. 1997). In contrast, such interaction can be neglected for the anti conformer up to moderate quantum numbers $\left(J+2 K_{a} \lesssim 30\right)$.

Pearson et al. (2008) reported extensively on the rotational spectra of the $\mathrm{C}_{2} \mathrm{H}_{5} \mathrm{OH}$ conformers and their mutual torsionrotation interaction. Data associated with this work as well as resulting predictions of the ground state rotational spectrum are available in the JPL catalog. Transition frequencies in the range of our ALMA survey are from that work. Vibrational contributions to the partition function at $150 \mathrm{~K}$ are dominated by the two torsional modes (Durig \& Larsen 1990). Combining anti and gauche conformers, the ground state partition function needs to be increased by a factor of 1.24; slight differences in the $\mathrm{OH}$ torsional energies of the two conformers are taken into account.

The entries of ethanol isotopologs with one ${ }^{13} \mathrm{C}$ atom were taken from the CDMS catalog. They were based on Bouchez et al. (2012), which restricted the investigations to the anti conformers with $J$ and $K_{a}$ ranges in which interaction with the gauche conformers can be neglected. The contribution of the gauche conformers to the partition function increases the value at $150 \mathrm{~K}$ based on the anti conformer only by a factor of $\sim 2.39$ as estimated in the CDMS documentation from the main isotopic 
Table 1. Experimental dipole moment components $\mu_{i}$ (D) of ethanol ${ }^{a}$ with proposed signs.

\begin{tabular}{llcc}
\hline \hline \multirow{2}{*}{ Component } & \multicolumn{2}{c}{ Value $^{a}$} & \multicolumn{2}{c}{ Sign $^{b}$} \\
\cline { 3 - 4 } & & Initial & Present \\
\hline$\mu_{a}(a \leftrightarrow a)$ & $0.046(14)$ & + & $(+)$ \\
$\mu_{b}(a \leftrightarrow a)$ & $1.438(7)$ & + & + \\
$\mu_{c}(a \leftrightarrow a)$ & $0 .^{c}$ & & \\
$\mu_{a}\left(g^{ \pm} \leftrightarrow g^{ \pm}\right)$ & $1.246(10)$ & - & + \\
$\mu_{b}\left(g^{ \pm} \leftrightarrow g^{ \pm}\right)$ & $0.104(8)$ & + & $(+)$ \\
$\mu_{c}\left(g^{ \pm} \leftrightarrow g^{\mp}\right)$ & $1.101(16)$ & - & - \\
\hline
\end{tabular}

Notes. ${ }^{(a)}$ From Takano et al. (1968) and Kakar \& Quade (1980) for anti and gauche conformers of ethanol, respectively. Numbers in parentheses after the values refer to the reported uncertainties in units of the least significant figures. $a, g^{+}$, and $g^{-}$refer to the anti and to the symmetric and antisymmetric states of the gauche conformers of ethanol, respectively. ${ }^{(b)}$ With respect to the signs of the interaction parameters in the Hamiltonian model from Pearson et al. (2008). Initial values are from that work. Signs in parentheses are not certain. See also the second to last paragraph in Sect. 2. ${ }^{(c)}$ By symmetry.
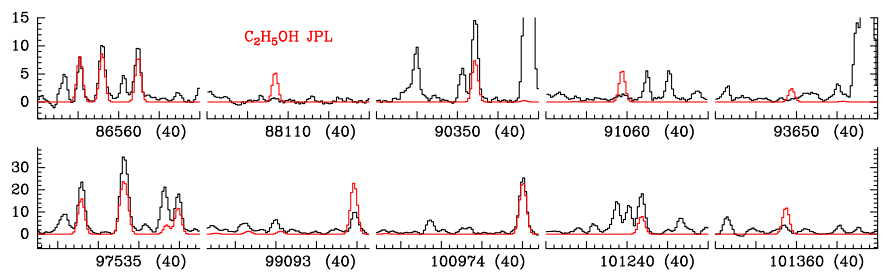

Fig. 1. Sections of the continuum-subtracted spectrum recorded with ALMA toward Sgr B2(N2) in black. The synthetic spectrum of ethanol using the official JPL catalog entry is shown in red. Some lines are predicted to be much stronger than they appear in the spectrum, e.g., near $88107 \mathrm{MHz}$ and $91059 \mathrm{MHz}$. A few other lines, e.g., near $90354 \mathrm{MHz}$ and $101244 \mathrm{MHz}$, are stronger than predicted. Compare with Fig. 2. The central frequency of each panel is indicated below the $x$-axis in $\mathrm{MHz}$; its width is given also in $\mathrm{MHz}$ in parentheses. The $y$-axis is labeled in brightness temperature units $(\mathrm{K})$.
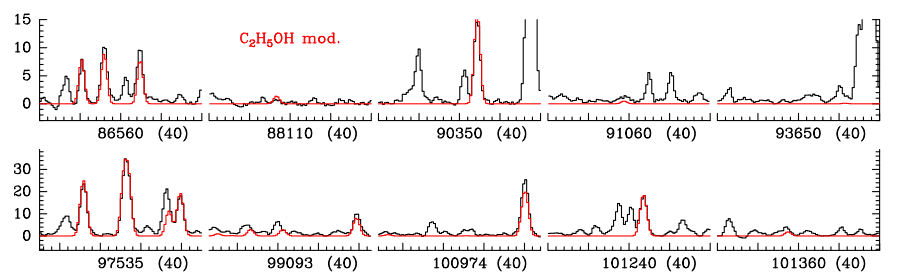

Fig. 2. Same as in Fig. 1 with the same LTE parameters, but the modeling was carried out with a modified catalog entry in which the sign of one dipole moment component was altered, see Table 1.

species. Vibrational contributions to the partition function were also derived from the main isotopic species.

The experimental dipole moment components of the ethanol conformers are given in Table 1 together with the proposed signs from Pearson et al. (2008), which are important because of the extensive torsion-rotation interaction. Pearson et al. (2008) discussed line strength issues in Sect. 5.2. They pointed out that intensities for a number of transitions are not correctly modeled by their Hamiltonian model and attributed this short-coming by the neglect of torsional corrections (sine and cosine functions) to the dipole moment components.
Table 2. Quantum numbers, conformers Conf., frequencies (MHz) of ethanol transitions appearing too strong in initial models, and Einstein $A$ values $\left(10^{-7} \mathrm{~s}^{-1}\right)$ from the JPL catalog and new values proposed in the present study.

\begin{tabular}{ccrcc}
\hline \hline$J^{\prime}, K_{a}^{\prime}, K_{c}^{\prime}-J^{\prime \prime}, K_{a}^{\prime \prime}, K_{c}^{\prime \prime}$ & Conf. & Frequency & $A(\mathrm{JPL})$ & $A(\mathrm{new})$ \\
\hline $28,10,18-29,9,20$ & $g \mp$ & 88106.42 & 5.55 & 6.26 \\
$28,10,19-29,9,21$ & $g \mp$ & 88106.57 & 5.55 & 6.26 \\
$26,0,26-25,1,24$ & $g \pm$ & 88106.96 & 22.76 & 2.82 \\
$25,2,24-24,3,21$ & $a$ & 91058.80 & 18.64 & 1.52 \\
$27,0,27-26,1,25$ & $g \pm$ & 93054.89 & 26.87 & 3.20 \\
$31,2,29-32,1,32$ & $a$ & 93648.78 & 23.57 & 0.18 \\
$28,0,28-27,1,26$ & $g \pm$ & 98097.68 & 31.55 & 3.60 \\
$13,0,13-13,1,13$ & $g \mp$ & 99109.25 & 68.67 & 16.37 \\
$13,4,9-12,5,7$ & $g \mp$ & 99109.69 & 7.04 & 7.11 \\
$19,3,16-19,2,18$ & $g \pm$ & 99537.22 & 34.23 & 11.36 \\
$24,2,23-23,3,20$ & $a$ & 99896.28 & 24.36 & 2.40 \\
$32,2,30-33,1,33$ & $a$ & 101134.52 & 29.75 & 0.21 \\
$15,2,13-15,1,15$ & $g \pm$ & 101357.44 & 36.42 & 5.16 \\
$29,1,29-28,2,27$ & $g \pm$ & 102326.79 & 16.70 & 3.93 \\
$29,0,29-28,1,27$ & $g \pm$ & 103215.29 & 36.82 & 4.03 \\
$30,0,30-29,1,28$ & $g \pm$ & 108392.97 & 42.71 & 4.29 \\
$33,2,31-34,1,34$ & $a$ & 108442.67 & 36.77 & 0.24 \\
$8,1,8-8,0,8$ & $g \mp$ & 110368.52 & 94.84 & 62.45 \\
$7,2,6-6,1,6$ & $g \pm$ & 110545.85 & 32.68 & 14.58 \\
$22,2,21-21,3,18$ & $a$ & 113309.00 & 34.73 & 5.15 \\
\hline
\end{tabular}

Notes. Transitions within the anti or trans conformer are indicated by $a, g \mp$ and $g \pm$ indicate transitions with gauche $^{+}$as lower and upper state, respectively, and gauche ${ }^{-}$as upper and lower state, respectively.

As shown in Fig. 1, we noticed that several rather weak $\mathrm{C}_{2} \mathrm{H}_{5} \mathrm{OH}$ transitions in our line survey were predicted to be significantly too strong; these transitions are summarized in Table 2. We suspected that the sign choices of the dipole moment components relative to the interaction parameters could be responsible for the observed intensity issues. We changed the signs of the dipole moment components from Pearson et al. (2008) one at a time relative to $\mu_{b}$ of the anti conformer. The resulting predictions were compared with our ALMA observations. In addition, we inspected a laboratory spectrum of ethanol in the $3 \mathrm{~mm}$ wavelength region taken at the Universität $\mathrm{zu}$ Köln for educational purposes. Sign changes of either $\mu_{a}$ or $\mu_{c}$ of the gauche conformer yielded predictions that were compatible with our ALMA observations with a slight preference for a change in $\mu_{a}$. The intensities in the laboratory spectrum were in support of this view; e.g., the gauche $e^{-}$transition $13_{1,12}-13_{1,13}$ near $96716.5 \mathrm{MHz}$ was about as strong as the $g^{+}-g^{-}$transi-

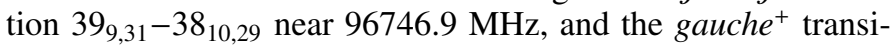
tion $13_{1,12}-13_{0,13}$ near $93279.0 \mathrm{MHz}$ was about twice as strong. Retaining the sign of $\mu_{a}$ and changing it in $\mu_{c}$ results in intensities of the two transitions with $J=13$ lower by a factor of about 2. Changing the signs of the dipole moment components that are small in magnitude, $\mu_{a}$ of the anti conformer and $\mu_{b}$ of the gauche conformer, had very little effect on the predicted intensities, as is expected. Therefore, we refrain from any statement concerning their signs. Figure 2 shows the modeling of ethanol with the altered sign of the dipole moment component. Not only are there now no weak transitions predicted to be much stronger than observed, in some cases the lines, for which initially only part of the observed signal was assigned to $\mathrm{C}_{2} \mathrm{H}_{5} \mathrm{OH}$, are now ascribed entirely to this molecule. Further details on the model are given in in Sect. 4.2; the entire model is presented in Appendix A. 
We should also point out that the choice of spectroscopic parameters may also affect the intensities. The intensities of $\Delta K=3$ transitions in the rotational spectrum of $\mathrm{PH}_{3}$ were discussed as a recent example (Müller 2013). Inclusion or omission of the $\Delta K=6$ term $h_{3}$ or fixing it to an estimated value has profound effects on the value of the $\Delta K=3$ term $\epsilon$ in the fit which, in turn, affects the intensities of the weak $\Delta K=3$ transitions. Pearson et al. (2008) discussed the determinability of odd order parameters (e.g., $G_{a}$ ) simultaneously with even order parameters (e.g., $F_{b c}$ ) although not explicitly in relation to intensities in the rotational spectrum of $\mathrm{C}_{2} \mathrm{H}_{5} \mathrm{OH}$.

We should emphasize that neglecting torsional corrections to the dipole moment components may also account for part of the intensity issues. Even though our dipole moment model was only studied in the $3 \mathrm{~mm}$ wavelength region, intensities may also be improved outside of this range. As indicated in this and the previous paragraphs, the proposed sign change does not necessarily resolve all intensity issues, even at $3 \mathrm{~mm}$. Inspection of the existing laboratory spectral recordings, in particular outside the $3 \mathrm{~mm}$ range, or temperature dependent intensity measurements, such as those carried out for methanol (Fortman et al. 2014; McMillan et al. 2014), may help resolve the intensity issues in the rotational spectrum of ethanol overall.

\subsection{Ethanethiol, $\mathrm{C}_{2} \mathrm{H}_{5} \mathrm{SH}$ : redetermination of spectroscopic parameters}

Schmidt \& Quade (1975) studied the microwave spectrum of ethanethiol, determined its dipole moment components, and estimated the anti conformer to have a higher energy than the gauche conformer by $204 \pm 22 \mathrm{~K}$. The spectroscopic analysis revealed gauche $e^{-}$to be higher than gauche ${ }^{+}$by only $1754 \mathrm{MHz}$ (or $0.084 \mathrm{~K}$ ). The dipole moment components are $\mu_{a}=1.48 \pm$ $0.02 \mathrm{D}$ and $\mu_{b}=0.19 \pm 0.10 \mathrm{D}$ within the gauche conformers and $\mu_{c}=0.59 \pm 0.02 \mathrm{D}$ between the gauche conformers. Values for the anti conformer are $\mu_{a}=1.06 \pm 0.03 \mathrm{D}$ and $\mu_{b}=1.17 \pm 0.03 \mathrm{D}$.

Far-infrared and Raman spectroscopy were also employed to evaluate the energy separation between the gauche and the anti conformer. Values of $144 \mathrm{~K}$ (Smith et al. 1973), $121 \mathrm{~K}$ (Inagaki et al. 1973), $177 \mathrm{~K}$ (Manocha et al. 1973), and $160 \mathrm{~K}$ (Durig et al. 1975) were obtained. The last value may be a reasonable compromise among these values and the one from microwave measurements, but it should be viewed with caution because the uncertainty is at least $30 \mathrm{~K}$, possibly more than $50 \mathrm{~K}$. An energy difference of $160 \mathrm{~K}$ yields a population of $17 \%$ for anti- $\mathrm{C}_{2} \mathrm{H}_{5} \mathrm{SH}$ at $180 \mathrm{~K}$, considering the ground vibrational states only. Combining the energy difference with the vibrational data from Durig et al. (1975), including in particular the differences in the SH torsional energies, the combined conformational and vibrational correction is $\sim 1.95$ at $180 \mathrm{~K}$.

Recently, Kolesniková et al. (2014) reported on the extension of the rotational spectrum up to 660 and $880 \mathrm{GHz}$ for the anti and gauche conformers of the main isotopic species, respectively, and on measurements of the gauche conformer of the ${ }^{34} \mathrm{~S}$ isotopic species up to $300 \mathrm{GHz}$. Their Hamiltonian employed for the gauche conformers was very similar to the one used earlier for the gauche conformers of ethanol (Pearson et al. 1996). These data were used to create entries for the CDMS. We noted that the use of odd and even order interaction parameters resulted in very unstable fits for the gauche conformers. Therefore, we tried to fit the data using a RAS, as described at the beginning of Sect. 2, i.e., we tried to avoid using odd order parameters, such as $G_{a}$ and $G_{b}$, in the fit. We note that these parameters were called $Q$ and $N$, respectively, by Kolesniková et al. (2014). The magnitudes of $15.0 \mathrm{MHz}$ and $6.3 \mathrm{MHz}$ determined for the parent species are much smaller than the theoretically limiting values of $\sim 2 A(57494 \mathrm{MHz})$ and $\sim 2 B(10590 \mathrm{MHz})$, respectively, suggesting that both parameters have only fairly small effects in the fit. It is worthwhile mentioning that Schmidt \& Quade (1975) did not use these parameters in their fits. In fact, they used $F_{b c}$ and $F_{a c}$ for their more limited data set, which they named $D_{+-}$and $E_{+-}$, respectively.

We determined spectroscopic parameters as averages between both states and expressed the differences as rotational corrections of the energy difference, as described above and as was done for ethanediol (Christen \& Müller 2003), for example. The advantage is that either parameter can be included in the fit without the other, whereas otherwise two distortion parameters are usually included in the fit, one for each state. We used Watson's $S$ reduction of the rotational Hamiltonian because it is more appropriate for asymmetric top rotors so close to the prolate case; $\kappa=(2 B-A-C) /(A-C) \approx-0.95$, close to the limiting case of -1 . We employed in our first fits only the rotational and quartic distortion parameters, as well as $E$ and $F_{b c}$. The last was expected to be very important as it connects the upper asymmetry component of gauche ${ }^{+}$with the lower asymmetry component of gauche $^{-}$with the same $J$ and $K_{a}$. The largest perturbations are expected if the asymmetry splitting is similar to the energy splitting between gauche $^{+}$and gauche ${ }^{-}$. We note that the energy difference is called $\Delta E$ in Kolesniková et al. (2014) and equals $2 E$ here by definition. At each stage of the fit, we searched among all reasonable parameters for the one that reduces the root mean square (rms) error as a measure of the quality of the fit most and which was determined with high significance. Neither $G_{a}$ nor $G_{b}$ was the parameter reducing the rms error most at any stage of the fit. Whenever one or the other was tried out in the fit, any appreciable reduction of the rms error made the fit highly unstable. This finding is hardly surprising as we do not have any indication that the transition frequencies of gauche- $\mathrm{C}_{2} \mathrm{H}_{5} \mathrm{SH}$ in the fit are affected by perturbation with the anti conformer. The final resulting parameters are given in Table 3.

We also determined new spectroscopic parameters for the gauche conformer of $\mathrm{C}_{2} \mathrm{H}_{5}^{34} \mathrm{SH}$. To this end, we took the parameters of the parent species and kept them fixed initially. Similarly to the fit of the parent species, we searched for the reasonable parameter whose floating in the fit caused the greatest reduction in the rms error until inclusion of new parameters caused only a minute reduction of the rms error. As some parameters showed larger isotopic changes than expected, we scaled the parameters, which were kept fixed, with appropriate powers of $B+C, B-C$, and $E$. The final set of spectroscopic parameters for $\mathrm{C}_{2} \mathrm{H}_{5}^{34} \mathrm{SH}$ are also given in Table 3.

In addition, we redetermined the spectroscopic parameters of anti- $\mathrm{C}_{2} \mathrm{H}_{5} \mathrm{SH}$. Kolesniková et al. (2014) used more centrifugal distortion parameters of eighth order for the anti conformer than for the gauche conformer even though fewer transition frequencies were determined extending to lower $J$ and, in particular, $K_{a}$ quantum numbers. We started with rotational and quartic centrifugal distortion parameters and weighted out lines that were difficult to reproduce. In the end, inclusion of only four additional sextic distortion parameters reproduced most of the transition frequencies well. The experimental line list is fairly sparse at higher $K_{a}$ values (9 to 15 ), and they were difficult to fit at somewhat higher $J$ (above 20). The residuals between observed and calculated frequencies showed only in some cases regular patterns. These residuals may be caused by perturbation of the rotational spectrum of the anti conformer or by blending 
Table 3. Spectroscopic parameters ${ }^{a}(\mathrm{MHz})$ of the gauche conformers of $\mathrm{C}_{2} \mathrm{H}_{5} \mathrm{SH}$ and $\mathrm{C}_{2} \mathrm{H}_{5}^{34} \mathrm{SH}$ determined in the present study.

\begin{tabular}{|c|c|c|}
\hline Parameter & $\mathrm{C}_{2} \mathrm{H}_{5} \mathrm{SH}$ & $\mathrm{C}_{2} \mathrm{H}_{5}^{34} \mathrm{SH}$ \\
\hline$A$ & $28746.9128(58)$ & $28708.8675(189)$ \\
\hline$B$ & 5294.877 932(99) & $5175.22138(36)$ \\
\hline$C$ & $4846.5753(58)$ & $4745.4191(72)$ \\
\hline$D_{K} \times 10^{3}$ & $203.82184(264)$ & $202.21(114)$ \\
\hline$D_{J K} \times 10^{3}$ & $-18.15294(89)$ & $-17.71601(185)$ \\
\hline$D_{J} \times 10^{3}$ & $3.287853(196)$ & $3.147245(116)$ \\
\hline$d_{1} \times 10^{6}$ & $-518.999(130)$ & $-485.551(133)$ \\
\hline$d_{2} \times 10^{6}$ & $-16.383(53)$ & $-14.889(87)$ \\
\hline$H_{K} \times 10^{6}$ & $5.4174(50)$ & 5.40 \\
\hline$H_{K J} \times 10^{6}$ & $-1.06141(106)$ & $-1.0290(72)$ \\
\hline$H_{J K} \times 10^{9}$ & $-3.18(35)$ & -3.0 \\
\hline$H_{J} \times 10^{9}$ & $2.66163(252)$ & 2.491 \\
\hline$h_{1} \times 10^{9}$ & $1.2091(90)$ & $1.0733(281)$ \\
\hline$h_{2} \times 10^{9}$ & $0.15598(200)$ & 0.140 \\
\hline$E$ & $876.99480(247)$ & $865.4782(62)$ \\
\hline$E_{K} \times 10^{3}$ & 71.894(308) & $64.73(238)$ \\
\hline$E_{J} \times 10^{3}$ & $-13.007(214)$ & $-10.926(248)$ \\
\hline$E_{2} \times 10^{3}$ & $-13.120(104)$ & $-11.948(118)$ \\
\hline$E_{K K} \times 10^{6}$ & $106.9(42)$ & 90. \\
\hline$E_{J K} \times 10^{6}$ & $-6.395(235)$ & -5.4 \\
\hline$E_{J J} \times 10^{9}$ & $84.5(84)$ & 69. \\
\hline$E_{K K K} \times 10^{9}$ & $-540.6(242)$ & -420 \\
\hline$E_{J K K} \times 10^{9}$ & $-8.31(185)$ & -6.4 \\
\hline$E_{J J K} \times 10^{9}$ & $0.469(41)$ & 0.36 \\
\hline$E_{K K K K} \times 10^{12}$ & 844.(41) & 610. \\
\hline$E_{J K K K} \times 10^{12}$ & $12.85(303)$ & 9.1 \\
\hline$F_{b c}$ & $12.20046(194)$ & $11.93560(118)$ \\
\hline$F_{b c, K} \times 10^{3}$ & $13.28(74)$ & 13.0 \\
\hline$F_{b c, J} \times 10^{6}$ & $93.0(47)$ & 91. \\
\hline$F_{b c, K K} \times 10^{6}$ & $6.70(43)$ & 6.6 \\
\hline$F_{b c, J J} \times 10^{9}$ & $-1.565(304)$ & -1.53 \\
\hline$F_{a c}$ & $100.95(69)$ & $100.33(86)$ \\
\hline$F_{a c, J} \times 10^{3}$ & $-0.951(39)$ & -0.944 \\
\hline$F_{2 a c} \times 10^{3}$ & $1.4683(100)$ & 1.458 \\
\hline
\end{tabular}

Notes. ${ }^{(a)}$ Watson's $S$ reduction was used in the representation $I^{r}$. Numbers in parentheses are one standard deviation in units of the least significant figures. Parameters of $\mathrm{C}_{2} \mathrm{H}_{5}^{34} \mathrm{SH}$ without uncertainties were derived from the main species and kept fixed in the fit.

of the assigned lines with other, unidentified lines. Therefore, we omitted these lines together with few lines having lower values of $K_{a}$ which were also weak for the most part. Predictions beyond $K_{a}=8$ or 9 should be viewed with caution because of these omissions. Transitions with such $K_{a}$ values may not be so important for astronomical observations because the anti conformer is considerably higher in energy than the gauche conformer. Our resulting spectroscopic parameters are presented in Table 4. Associated line, parameter, and fit files along with additional auxiliary files will be available in the Fitting Spectra section of the $\mathrm{CDMS}^{4}$.

The experimental transition frequencies of the gauche conformers of the parent and ${ }^{34} \mathrm{~S}$ species of ethanethiol have been reproduced to $39.6 \mathrm{kHz}$ and $38.1 \mathrm{kHz}$, respectively, about the

\footnotetext{
4 http://www .astro.uni-koeln.de/site/vorhersagen/ pickett/beispiele/EtSH/
}

Table 4. Spectroscopic parameters ${ }^{a}(\mathrm{MHz})$ of anti- $\mathrm{C}_{2} \mathrm{H}_{5} \mathrm{SH}$ determined in the present study.

\begin{tabular}{lc}
\hline \hline Parameter & Value \\
\hline$A$ & $28416.76803(214)$ \\
$B$ & $5485.766323(169)$ \\
$C$ & $4881.832621(127)$ \\
$D_{K} \times 10^{3}$ & $211.166(149)$ \\
$D_{J K} \times 10^{3}$ & $-22.1853(58)$ \\
$D_{J} \times 10^{3}$ & $3.787500(119)$ \\
$d_{1} \times 10^{6}$ & $-658.795(45)$ \\
$d_{2} \times 10^{6}$ & $-23.555(46)$ \\
$H_{K} \times 10^{6}$ & $108.19(267)$ \\
$H_{J K} \times 10^{9}$ & $-300.6(63)$ \\
$h_{2} \times 10^{9}$ & $0.7740(234)$ \\
$h_{3} \times 10^{9}$ & $0.2538(158)$ \\
\hline
\end{tabular}

Notes. ${ }^{(a)}$ Watson's $S$ reduction was used in the representation $I^{r}$.

same as in Kolesniková et al. (2014), where the rms values were $39 \mathrm{kHz}$ and $41 \mathrm{kHz}$. However, we only required 34 parameters for the parent species whereas Kolesniková et al. (2014) used 41. In the case of the ${ }^{34} \mathrm{~S}$ isotopic species, 16 versus 19 parameters were floated. Therefore, we expect our parameters to describe the rotational spectrum better. Predictions much beyond the covered quantum number range $\left(J \leq 88, K_{a} \leq 25\right.$ for the parent species) should be viewed with caution. Such transitions are likely well beyond the scope of astronomical observations.

We looked into intensity alterations dependent on sign changes of the dipole moment components and found these alterations to be much smaller and much rarer than in ethanol (see Sect. 2.3). Nevertheless, predicted intensities should be viewed with some caution.

Our values of $E_{K K}, E_{K K K}$, and $E_{K K K K}$ are fairly large in magnitude, possibly indicating perturbations of the spectrum by its lowest vibrational mode or by the anti conformer. Schmidt \& Quade (1975) determined $F_{b c}=12.20 \mathrm{MHz}$ and $F_{a c}=$ 139.34 MHz. The former agrees perfectly with our value, and the agreement for the latter is reasonable if we take into account that in our case the uncertainty is $0.69 \mathrm{MHz}$ and in the case of Schmidt \& Quade (1975) it is at least several megahertz.

We have also determined spectroscopic parameters of the anti conformer using Watson's $A$ reduction for comparison purposes with parameters of Kolesniková et al. (2014) and with values from quantum chemical calculations (Puzzarini et al. 2014). These parameters are given in Table 5.

The two sets of experimental rotational and quartic centrifugal distortion parameters agree well, the small, but significant differences in $\Delta_{K}$ and $\delta_{K}$ are caused by larger differences between the higher order parameters. The agreement is also good for values calculated by quantum chemical means. The rotational parameters include first-order vibrational corrections and are commonly marginally larger than the experimental ones at that level of computation. Quantum chemically calculated distortion parameters usually do not include vibrational corrections. The deviations between calculated quartic centrifugal distortion parameters and the experimental values are largely caused by this neglect. The agreement between the two sets of experimental parameters is moderate to poor for the few sextic distortion parameters used in the present fit. The very large $L_{K}$ value in Kolesniková et al. (2014) is largely responsible for the much 
Table 5. Spectroscopic parameters (MHz) of anti- $\mathrm{C}_{2} \mathrm{H}_{5} \mathrm{SH}$ in Watson's $A$ reduction from the present study in comparison to previous experimental values ${ }^{a}$ and those from quantum-chemical (QC) calculations ${ }^{b}$.

\begin{tabular}{lccc}
\hline \hline Parameter & Present exptl. & Previous exptl. $^{a}$ & QC values $^{b}$ \\
\hline$A$ & $28416.7769(21)$ & $28416.7604(18)$ & 28451.975 \\
$B$ & $5485.77873(28)$ & $5485.77901(15)$ & 5489.912 \\
$C$ & $4881.81782(17)$ & $4881.81762(12)$ & 4885.345 \\
$\Delta_{K} \times 10^{3}$ & $211.35(15)$ & $210.03(18)$ & 198.873 \\
$\Delta_{J K} \times 10^{3}$ & $-22.3951(48)$ & $-22.4549(58)$ & -23.107 \\
$\Delta_{J} \times 10^{3}$ & $3.83086(36)$ & $3.83217(27)$ & 3.767 \\
$\delta_{K} \times 10^{3}$ & $7.246(15)$ & $7.342(20)$ & 5.967 \\
$\delta_{J} \times 10^{3}$ & $0.65725(19)$ & $0.65664(11)$ & 0.555 \\
$\Phi_{K} \times 10^{6}$ & $103.7(27)$ & $26.7(80)$ & -5.4647 \\
$\Phi_{K J} \times 10^{6}$ & & $-2.717(106)$ & 1.0607 \\
$\Phi_{J K} \times 10^{9}$ & & $367 .(18)$ & -42.3 \\
$\Phi_{J} \times 10^{9}$ & $-1.36(28)$ & $-0.86(17)$ & -13.0 \\
$\phi_{K} \times 10^{6}$ & $13.72(31)$ & $28.89(97)$ & -1.3450 \\
$\phi_{J K} \times 10^{9}$ & $80 .(14)$ & $-164 .(26)$ & -226.5 \\
$\phi_{J} \times 10^{9}$ & $-1.16(14)$ & $-0.935(91)$ & -6.4 \\
$L_{K} \times 10^{6}$ & & $1.374(110)$ & \\
$L_{K K J} \times 10^{9}$ & & $2.11(40)$ & \\
$L_{J} \times 10^{15}$ & & $60 .(11)$ & \\
$l_{K J} \times 10^{9}$ & & $4.55(50)$ & \\
\hline
\end{tabular}

Notes. ${ }^{(a)}$ Kolesniková et al. (2014). ${ }^{(b)}$ Puzzarini et al. (2014); highest level CCSD(T)/cc-pVTZ values.

smaller value of $\Phi_{K}$. In fact, contributions of $L_{K}$ are larger than those of $\Phi_{K}$ already at $K_{a}=5$. The agreement is also poor between either set of sextic distortion parameters and those derived from quantum chemical calculations. Puzzarini et al. (2014) attributed these deviations entirely to errors in the experimental values. However, all three values (gauche- and antiethanethiol and dimethyl sulfide) of $\Phi_{K} \approx H_{K}$ were reported as negative in that work. This is very unusual for an asymmetric top rotor close to the prolate limit, which usually has a positive value. For example, $\sim 23$ and $\sim 18 \mathrm{~Hz}$ were obtained for the anti conformers of the two isotopomers of ethanol with one ${ }^{13} \mathrm{C}$ (Bouchez et al. 2012), $\sim 13.5 \mathrm{~Hz}$ for dimethyl ether with 0 (Endres et al. 2009), 1, and $2{ }^{13} \mathrm{C}$ atoms (Koerber et al. 2013), and $93 \mathrm{~Hz}$ for propargyl alcohol (Pearson \& Drouin 2005). Therefore, we suspect that there are errors in the quantum chemical calculations of sextic distortion parameters in Puzzarini et al. (2014).

\section{5. normal-Propanol, $n-\mathrm{C}_{3} \mathrm{H}_{7} \mathrm{OH}$}

Replacing one $\mathrm{H}$ atom at the terminal $\mathrm{C}$ atom in ethanol with a $\mathrm{CH}_{3}$ group yields the unbranched $n$-propanol. There are three possibilities for doing so with CCCO dihedral angles of $180^{\circ}$ or $\pm 60^{\circ}$. As in the case of the HOCC dihedral, the conformations are called anti (or trans) and gauche, the latter being doubly degenerate. Orientations of the methyl group are designated by upper-case characters, those of the $\mathrm{OH}$ group by lower-case characters. The $G a$ conformer is lowest in energy, and the two non-equivalent $G g$ and $G g$ ' conformers are higher by $68.81 \mathrm{~K}$ and $73.17 \mathrm{~K}$, respectively, as determined from perturbations in rotational transitions of $G a$ at high $K_{a}$ quantum numbers and from perturbations between $G g$ and $G g$ ' (Kisiel et al. 2010). The energy of the $A a$ conformer relative to $G a$ is known only approximately, but it appears to be lower than that of either $G g$ and $G g^{\prime}$ (Kahn \& Bruice 2005). The doubly degenerate $\mathrm{Ag}$ conformer is also quite close in energy to the other conformers (Kahn \& Bruice 2005), and tunneling between the two equivalent conformers will likely lead to two distinguishable $\mathrm{Ag}^{+}$and $\mathrm{Ag}^{-}$states. Uncertainties of the relative energies of the $A$ conformers are difficult to estimate, but are probably at least $10 \mathrm{~K}$ if not a multiple thereof.

Predictions of the rotational spectrum of $G a$-n-propanol were taken from the CDMS. The entry is based on the extensive data from Maeda et al. (2006a) with experimental data almost exclusively from that work. Frequencies of perturbed transitions were omitted. These transitions all have rather high values of $K_{a}$ and will be of importance for astronomical observations only if lower energy transitions have been detected with very high signal-to-noise ratios and at high rotational temperatures. Dipole moment components of $\mu_{a}=0.4914$ (4) D, $\mu_{b}=0.9705$ (13) D, and $\mu_{c}=0.9042$ (12) D were determined by Kisiel et al. (2010) from a single state fit. Based on the relative energies of the $n$ propanol conformers, their total column density in the ground vibrational state at $150 \mathrm{~K}$ is about a factor of 3.3 higher than that determined for the $G a$ conformer; inclusion of thermal population of vibrational states (Fukushima \& Zwolinski 1968) yields a combined factor of about 5.2.

\section{6. iso-Propanol, $\mathrm{i}-\mathrm{C}_{3} \mathrm{H}_{7} \mathrm{OH}$}

Replacing one $\mathrm{H}$ atom at the central $\mathrm{C}$ atom in ethanol by a $\mathrm{CH}_{3}$ group yields the branched $i$-propanol. The two positions are equivalent. There are again three orientations for the $\mathrm{OH}$ group: anti (or trans) and the doubly degenerate gauche. Tunneling between the two equivalent gauche conformers leads again to gauche $^{+}$and gauche ${ }^{-}$, which are separated by $2.236 \mathrm{~K}$ (Maeda et al. 2006b). There is consensus that the anti conformer is higher in energy than the gauche conformers, but the amount differs considerably. Intensity measurements at millimeter wavelengths (Maeda et al. 2006b) yielded a difference of $\sim 120 \mathrm{~K}$ with an estimated uncertainty of $15 \mathrm{~K}$. This value is in reasonable agreement with $81 \mathrm{~K}$ (Kahn \& Bruice 2005) and with $227 \pm 104 \mathrm{~K}$ from intensity measurements in the microwave region (Hirota 1979). A value of $12.5 \mathrm{~K}$ (Inagaki et al. 1973) appears to be too small. We have employed an energy difference of $100 \mathrm{~K}$ as the average of the two determinations judged to be most reliable. At $150 \mathrm{~K}$ rotational temperature, this leads to a column density correction factor of 1.26 from thermal population of the anti conformer. Taking into account vibrational data from Inagaki et al. (1973) and Dobrowolski et al. (2008) yields a combined conformational and vibrational correction factor to the partition function of $\sim 1.86$.

Predictions were generated from data summarized in Maeda et al. (2006b), which are based largely on measurements from that work covering most of the 115-360 GHz region. Hirota (1979) determined $\mu_{a}=1.114$ (15) D between the gauche substates and $\mu_{b}=0.737$ (25) D and $\mu_{c}=0.8129$ (49) D within the gauche substates.

\section{Observations and data reduction}

Part of the observations used in this article have been briefly described in Belloche et al. (2014). A detailed account of the observations, reduction, and analysis method of the full data set is reported in a companion paper (Belloche et al. 2016). 


\section{Results}

\subsection{Methanol $\mathrm{CH}_{3} \mathrm{OH}$}

Methanol is well detected toward Sgr B2(N2) in its vibrational ground state and in its first torsionally excited state $v_{\mathrm{t}}=1$ (Figs. A.1 and A.2). The second torsionally excited state is relatively well detected as well, with about three detected lines and seven additional lines that contribute significantly to the detected signal (Fig. A.3). The six lines between 96.19 and $96.56 \mathrm{GHz}$ are the $a$-type $(\Delta K=0)$ transitions with $J=2-1$. The remaining features are $b$-type transitions with $J \leq 21$. The third torsionally excited state $\left(v_{\mathrm{t}}=3\right)$ cannot be considered as unambiguously detected; however, since it contributes significantly to the flux detected at $96268 \mathrm{MHz}\left(2_{1}-1_{1}\right.$ of $E$ symmetry) according to our model that uses the same parameters as for the lower states, we include it in the full model (Fig. A.4).

The ${ }^{13} \mathrm{C}$ isotopolog of methanol is also clearly detected, both in its ground state and in $v_{\mathrm{t}}=1$ (Figs. A.5 and A.6). Some lines in the ground state are marginally optically thick $\left(\tau_{\max }=1.3\right)$. About eight lines of the ${ }^{18} \mathrm{O}$ isotopolog are detected in its vibrational ground state, which makes the assignment secure (Fig. A.7). The $J=2-1 a$-type transitions occur at $91958 \mathrm{MHz}$ (not shown, strongly blended), around $92730 \mathrm{MHz}$, and at $93506 \mathrm{MHz}$. The remainder are $b$-type transitions with low values of $K$ and low to moderate values of $J$. Their emission is optically thin $\left(\tau_{\max }=0.14\right)$. Transitions from within $v_{\mathrm{t}}=1$ are not unambiguously detected for this isotopolog, but its emission contributes significantly to the line detected at $97069 \mathrm{MHz}$ (Fig. A.8) which is why we included it in the full model. Our model allows us to derive the following isotopic ratios for methanol: ${ }^{12} \mathrm{C} /{ }^{13} \mathrm{C} \approx 25$ and ${ }^{16} \mathrm{O} /{ }^{18} \mathrm{O} \approx 180$. The ${ }^{13} \mathrm{CH}_{3} \mathrm{OH} / \mathrm{CH}_{3}^{18} \mathrm{OH}$ ratio is $\sim 7.3$.

The fits to the integrated intensity maps of the detected methanol lines yield a source size that decreases with increasing energy level, from $\sim 1.4^{\prime \prime}$ at low energy to $\sim 1.0^{\prime \prime}$ at $E_{\text {up }} \approx 1000 \mathrm{~K}$. A similar behavior is found for the ${ }^{13} \mathrm{C}$ isotopolog over a narrower energy range. The population diagrams of methanol (restricted to its transitions with an opacity lower than 2) and its isotopologs are very well fitted with a rotation temperature of about 140-160 K (Table 6 and Figs. A.9-A.11). The rotation temperature of the $\mathrm{CH}_{3} \mathrm{OH}$ ground vibrational state is constrained at low to moderate energies, for example, by the $J_{-2}-J_{1}$ transitions of $E$ symmetry. The lines are very weak at low $J(J=2-4$ near $101.1 \mathrm{GHz}$ ), but have substantial opacities at higher $J ; J=17$ at $111626.5 \mathrm{MHz}$ is the last line in the region of our survey. On the higher energy side are the two asymmetry split $26_{5}-25_{6}$ transitions of $A$ symmetry around $97210 \mathrm{MHz}$. The spectra of the optically thick lines of methanol require a large size and high temperature to be well fitted. Our LTE model thus assumes a source size of $1.4^{\prime \prime}$ and a rotational temperature of $160 \mathrm{~K}$. The fit is good for most lines of all three isotopologs, except for the shape of the very optically thick lines of methanol (with $\tau$ up to 19) and the likely masing lines at 84521 and $95169 \mathrm{MHz}\left(5_{-1}-4_{0}\right.$ of $E$ symmetry and $88_{0}-7_{1}$ of $A^{+}$symmetry, respectively; see, e.g., Müller et al. 2004).

\subsection{Ethanol $\mathrm{C}_{2} \mathrm{H}_{5} \mathrm{OH}$}

About 170 lines of ethanol are detected toward Sgr B2(N2) in its vibrational ground state (Fig. A.12). The fits to the integrated intensity maps of these lines yield a median source size of $\sim 1.3^{\prime \prime}$, with no clear trend as a function of upper-level energy. The population diagram shown in Fig. A.13 yields a well-constrained
Table 6. Rotational temperatures derived from population diagrams of alkanols and alkanethiols toward Sgr B2(N2).

\begin{tabular}{lll}
\hline \hline Molecule & \multicolumn{1}{c}{ States $^{a}$} & \multicolumn{1}{c}{$\begin{array}{c}T_{\text {fit }}{ }^{b} \\
(\mathrm{~K})\end{array}$} \\
\hline $\mathrm{CH}_{3} \mathrm{OH}$ & $v=0, v_{\mathrm{t}}=1, v_{\mathrm{t}}=2$ & $150.8(1.7)$ \\
${ }^{13} \mathrm{CH}_{3} \mathrm{OH}$ & $v=0, v_{\mathrm{t}}=1$ & $160.6(6.8)$ \\
$\mathrm{CH}_{3}{ }^{18} \mathrm{OH}$ & $v=0$ & $143(12)$ \\
\hline $\mathrm{C}_{2} \mathrm{H}_{5} \mathrm{OH}$ & $v=0$ & $139.6(1.6)$ \\
\hline $\mathrm{CH}_{3} \mathrm{SH}$ & $v=0, v_{\mathrm{t}}=1$ & $208(46)$ \\
\hline
\end{tabular}

Notes. ${ }^{(a)}$ Vibrational states that were taken into account to fit the population diagram. ${ }^{(b)}$ The standard deviation of the fit is given in parentheses. As explained in Belloche et al. (2016), these uncertainties should be viewed with caution. They may be underestimated.

rotational temperature (Table 6). We use a slightly higher temperature $(150 \mathrm{~K}$ versus $140 \mathrm{~K})$, still fully consistent with the intensities of the detected lines. With this source size and temperature, some lines turn out to be marginally optically thick $\left(\tau_{\max }=1.2\right)$

Both ${ }^{13} \mathrm{C}$ isotopologs of ethanol are detected in their anti form with a few clearly detected lines each (Figs. A.14 and A.15). We derive a ${ }^{12} \mathrm{C} /{ }^{13} \mathrm{C}$ isotopic ratio of $\sim 25$ for ethanol, consistent with the one derived for methanol (see Sect. 4.1).

\subsection{Propanol normal- $\mathrm{C}_{3} \mathrm{H}_{7} \mathrm{OH}$ and iso $-\mathrm{C}_{3} \mathrm{H}_{7} \mathrm{OH}$}

Propanol is not detected toward Sgr B2(N2) either in its straight chain form (normal) or in its branched form (iso). Assuming the same parameters as for ethanol, we derive upper limits to the column density of both forms (Table 7).

\subsection{Methanethiol $\mathrm{CH}_{3} \mathrm{SH}$}

With about 12 lines clearly detected in its vibrational ground state, methanethiol is securely identified toward Sgr B2(N2) (Fig. A.16). We also identified torsionally excited transitions $\left(v_{\mathrm{t}}=1\right)$ belonging to the $a$-type $J=4-3$ branch, which are shown in Fig. A.17. Even though they are slightly or heavily blended, two almost completely blended transitions, $k=+1$ of $E$ and $4_{2,3}-3_{2,2}$ of $A$ torsional symmetry near $100806 \mathrm{MHz}$, account for essentially all of that emission feature. In addition, $k=+2$ near $100838 \mathrm{MHz}$ and $4_{1,3}-3_{1,2}$ of $A$ torsional symmetry near $101522 \mathrm{MHz}$ account for emission features that are part of a blend. The $v_{\mathrm{t}}=1$ state is not unambiguously detected; however, since the synthetic spectrum computed with the same parameters as for the ground state is fully consistent with the observed spectrum, we consider this state as tentatively detected and we include it in the full model (Fig. A.17). The fits to the integrated intensity maps of the detected ground state lines yield a source size of $\sim 1.4^{\prime \prime}$. The fit to the population diagram including both states is not well constrained $\left(T_{\text {rot }}=210 \pm 50 \mathrm{~K}\right.$, Table 6 and Fig. A.18). This fit is dominated by the more numerous lines in the vibrational ground state and tends to overestimate the intensities of the lines in the first vibrationally excited state. To avoid this overestimate when modeling the spectrum, we use a lower temperature of $180 \mathrm{~K}$, still consistent with the fit result above. With this source size and temperature, the emission is optically thin $\left(\tau_{\max }=0.3\right)$. The apparent discrepancy between the synthetic and observed spectra at $108680 \mathrm{MHz}$ is likely due to ${ }^{13} \mathrm{CN}$ absorption produced by diffuse clouds along the line of 
Table 7. Parameters of our best-fit LTE model (or upper limit) of alkanols and alkanethiols toward Sgr B2(N2).

\begin{tabular}{|c|c|c|c|c|c|c|c|c|c|}
\hline Molecule & Status $^{a}$ & $N_{\operatorname{det}^{b}}$ & $\begin{array}{r}\operatorname{Size}^{c} \\
\left({ }^{\prime \prime}\right)\end{array}$ & $\begin{array}{r}T_{\text {rot }}{ }^{d} \\
(\mathrm{~K})\end{array}$ & $\begin{array}{c}N^{e} \\
\left(\mathrm{~cm}^{-2}\right)\end{array}$ & $C^{f}$ & $\begin{array}{c}\Delta V^{g} \\
\left(\mathrm{~km} \mathrm{~s}^{-1}\right)\end{array}$ & $\begin{array}{c}V_{\text {off }^{h}} \\
\left(\mathrm{~km} \mathrm{~s}^{-1}\right)\end{array}$ & $\frac{N_{\text {ref }}}{N} i$ \\
\hline $\mathrm{CH}_{3} \mathrm{OH}, v=0^{\star}$ & $\mathrm{d}$ & 41 & 1.4 & 160 & 4.0 (19) & 1.00 & 5.4 & -0.5 & 1 \\
\hline$v_{\mathrm{t}}=1$ & $\mathrm{~d}$ & 16 & 1.4 & 160 & $4.0(19)$ & 1.00 & 5.4 & -0.2 & 1 \\
\hline$v_{\mathrm{t}}=2$ & $\mathrm{~d}$ & 3 & 1.4 & 160 & 4.0 (19) & 1.00 & 5.4 & -0.5 & 1 \\
\hline$v_{t}=3$ & $\mathrm{t}$ & 0 & 1.4 & 160 & 4.0 (19) & 1.00 & 5.4 & -0.5 & 1 \\
\hline${ }^{13} \mathrm{CH}_{3} \mathrm{OH}, v=0$ & $\mathrm{~d}$ & 19 & 1.4 & 160 & $1.6(18)$ & 1.00 & 5.4 & -0.2 & 25 \\
\hline$v_{\mathrm{t}}=1$ & $\mathrm{~d}$ & 7 & 1.4 & 160 & $1.6(18)$ & 1.00 & 5.4 & -0.2 & 25 \\
\hline $\mathrm{CH}_{3}{ }^{18} \mathrm{OH}, v=0$ & $\mathrm{~d}$ & 8 & 1.4 & 160 & $2.2(17)$ & 1.00 & 5.4 & -0.0 & 182 \\
\hline$v_{\mathrm{t}}=1$ & $\mathrm{t}$ & 0 & 1.4 & 160 & $2.2(17)$ & 1.00 & 5.4 & -0.0 & 182 \\
\hline $\mathrm{C}_{2} \mathrm{H}_{5} \mathrm{OH}^{\star}$ & $\mathrm{d}$ & 168 & 1.3 & 150 & $2.0(18)$ & 1.24 & 5.4 & 0.0 & 1 \\
\hline anti- ${ }^{13} \mathrm{CH}_{3} \mathrm{CH}_{2} \mathrm{OH}$ & $\mathrm{d}$ & 4 & 1.3 & 150 & $8.0(16)$ & 2.96 & 5.4 & 0.0 & 25 \\
\hline anti- $\mathrm{CH}_{3}{ }^{13} \mathrm{CH}_{2} \mathrm{OH}$ & d & 3 & 1.3 & 150 & $8.0(16)$ & 2.96 & 5.4 & 0.0 & 25 \\
\hline Ga-normal- $\mathrm{C}_{3} \mathrm{H}_{7} \mathrm{OH}$ & $\mathrm{n}$ & 0 & 1.3 & 150 & $<2.6(17)$ & 5.20 & 5.4 & 0.0 & - \\
\hline iso- $\mathrm{C}_{3} \mathrm{H}_{7} \mathrm{OH}$ & $\mathrm{n}$ & 0 & 1.3 & 150 & $<9.3(16)$ & 1.86 & 5.4 & 0.0 & - \\
\hline $\mathrm{CH}_{3} \mathrm{SH}, v=0^{\star}$ & $\mathrm{d}$ & 12 & 1.4 & 180 & $3.4(17)$ & 1.00 & 5.4 & -0.5 & 1 \\
\hline$v_{\mathrm{t}}=1$ & $\mathrm{t}$ & 1 & 1.4 & 180 & 3.4 (17) & 1.00 & 5.4 & -0.5 & 1 \\
\hline gauche $-\mathrm{C}_{2} \mathrm{H}_{5} \mathrm{SH}$ & $\mathrm{n}$ & 0 & 1.4 & 180 & $<1.6(16)$ & 1.95 & 5.4 & -0.5 & - \\
\hline
\end{tabular}

Notes. ${ }^{(a)}$ d: detection, t: tentative detection, n: non-detection. ${ }^{(b)}$ Number of detected lines (conservative estimate, see Belloche et al. 2016). Here, one line of a given species may mean a group of transitions of that species that are blended together. ${ }^{(c)}$ Source diameter (FWHM). ${ }^{(d)}$ Rotational temperature. ${ }^{(e)}$ Total column density of the molecule. $X(Y)$ means $X \times 10^{Y} .{ }^{(f)}$ Correction factor that was applied to the column density to account for the contribution of vibrationally or torsionally excited states or other conformers, in the cases where this contribution was not included in the partition function of the spectroscopic predictions. ${ }^{(g)}$ Linewidth (FWHM). ${ }^{(h)}$ Velocity offset with respect to the assumed systemic velocity of Sgr B2(N2) $V_{\mathrm{lsr}}=74 \mathrm{~km} \mathrm{~s}^{-1}$. ${ }^{(i)}$ Column density ratio, with $N_{\text {ref }}$ the column density of the previous reference species marked with a $\star$.

sight, which we have not completely modeled so far. The small discrepancy at $110342 \mathrm{MHz}$ may be due to an overestimate of the level of the baseline. This frequency corresponds to the data point significantly below the model at $E_{\mathrm{u}} / k_{\mathrm{b}} \approx 100 \mathrm{~K}$ (in red) in the population diagram.

\subsection{Ethanethiol $\mathrm{C}_{2} \mathrm{H}_{5} \mathrm{SH}$}

Ethanethiol is not unambiguously detected toward Sgr B2(N2). Under the assumption of the same temperature, source size, linewidth, and velocity offset as for methanethiol (Sect. 4.4), the synthetic spectrum shown in Fig. A.19 is consistent with the observed spectrum, especially for the $11_{4}-10_{4}$ multiplet near $111647 \mathrm{MHz}$, but the lack of clearly detected lines prevents a secure identification. We consider this model as a $3 \sigma$ upper limit to the column density of ethanethiol toward Sgr B2(N2) (Table 7).

\section{Astrochemical modeling}

In order to investigate the chemical formation and destruction mechanisms associated with $\mathrm{CH}_{3} \mathrm{SH}$ and $\mathrm{C}_{2} \mathrm{H}_{5} \mathrm{SH}$, we incorporate a new set of reactions into the network presented by Belloche et al. (2014). This expanded network is used to simulate the coupled gas-phase and grain-surface chemistry occurring in Sgr B2(N2), using the kinetics model MAGICKAL (Garrod 2013).

Current astrochemical networks include relatively few sulfur-bearing molecules; with reference to the production of alkanethiols, none of them (to the authors' knowledge) includes any treatment for the formation of sulfur-bearing molecules larger than $\mathrm{H}_{2} \mathrm{CS}$. Here, in analogy with the dominant formation routes for methanol and ethanol in hot cores, as predicted by the chemical models, the new network concentrates on the production of alkanethiols solely on the surfaces of the dust grains. However, gas-phase destruction mechanisms for both molecules (as well as related intermediates) are included in the new network, the majority of which are ion-molecule processes or the subsequent dissociative recombination with electrons of the resultant molecular ions. Ion-molecule reactions are included for the major ionic species $\mathrm{He}^{+}, \mathrm{H}^{+}, \mathrm{H}_{3}{ }^{+}, \mathrm{H}_{3} \mathrm{O}^{+}$and $\mathrm{HCO}^{+}$. Estimates for the rates of photo-dissociation of new molecules, as caused by cosmic ray-induced and (where extinction allows) external UV photons, are also included (see Garrod et al. 2008 and Garrod 2013).

For $\mathrm{CH}_{3} \mathrm{SH}$, we assume the same grain-surface desorption/binding energy as for methanol $(5534 \mathrm{~K})$, which is based on the laboratory study of Collings et al. (2004). The weak hydrogen-bonding character of the thiol group is likely to result in a somewhat lower binding energy for methanethiol than for methanol in the amorphous water-dominated ices present on the dust grains; however, based on the greater observed rotational temperature for $\mathrm{CH}_{3} \mathrm{SH}$ than for $\mathrm{CH}_{3} \mathrm{OH}$ observed in our source $(180 \mathrm{~K}$ versus $150 \mathrm{~K})$, indicating a similar or greater desorption temperature for $\mathrm{CH}_{3} \mathrm{SH}$, the adoption of the methanol-based value appears justified in the absence of appropriate laboratory data. In the case of $\mathrm{C}_{2} \mathrm{H}_{5} \mathrm{SH}$, a binding energy of $6230 \mathrm{~K}$ is used - based on interpolation as detailed by Garrod (2013) - that is close to the value already adopted for ethanol (6259 K).

Methanethiol may form on grain surfaces through the addition of hydrogen atoms to $\mathrm{H}_{2} \mathrm{CS}$. Both gas-phase and grainsurface formation routes already exist in the network for CS, $\mathrm{HCS}$, and $\mathrm{H}_{2} \mathrm{CS}$. The new network thus includes a chain of hydrogen addition reactions analogous to those for methanol, accounting for all hydrogenation states: CS, $\mathrm{HCS}, \mathrm{H}_{2} \mathrm{CS}$, $\mathrm{CH}_{3} \mathrm{~S} / \mathrm{CH}_{2} \mathrm{SH}$ and $\mathrm{CH}_{3} \mathrm{SH}$. Methanethiol may also be formed via the addition of $\mathrm{CH}_{3}$ and $\mathrm{HS}$, and other species in the chain may also be formed via alternative atomic additions, e.g., $\mathrm{CH}_{2}+$ $\mathrm{S} \rightarrow \mathrm{H}_{2} \mathrm{CS}$. Ethanethiol may be formed by a selection of radicalradical and hydrogen-addition reactions on the grains, including $\mathrm{CH}_{3}+\mathrm{CH}_{2} \mathrm{SH}$ and $\mathrm{C}_{2} \mathrm{H}_{5}+\mathrm{HS}$. 
The physical model follows that detailed in previous papers where a cold collapse phase to maximum density $\left(n_{\mathrm{H}}=2 \times 10^{8} \mathrm{~cm}^{-3}\right)$ and minimum dust-grain temperature $(8 \mathrm{~K})$ is followed by a warm-up from $8-400 \mathrm{~K}$; during this phase, the gas and dust temperatures are assumed to be well coupled.

The initial chemical compositions used in the model follow those of Garrod (2013). Crucially, a standard, depleted sulfur abundance of $8 \times 10^{-8}$ is used, which reproduces well the relative abundances of the sulfur-bearing molecules. The underlying reason for the anomalous depletion of atomic sulfur in dense regions (as compared to diffuse cloud values) is a long-standing problem (e.g., Anderson et al. 2013), and will not be addressed here.

\subsection{Results of modeling}

Figure 3 shows the results of the models, for $\mathrm{CH}_{3} \mathrm{SH}, \mathrm{C}_{2} \mathrm{H}_{5} \mathrm{SH}$, and a selection of related molecules (a), and their oxygensubstituted equivalents, each of which is a well-known hot-core molecule (b). The plots indicate the calculated time-dependent abundances of each molecule with respect to molecular hydrogen, during the warm-up phase of the chemistry. Solid lines indicate gas-phase abundances, while dotted lines of the same color indicate ice-mantle abundances for the same species.

Most of the $\mathrm{CH}_{3} \mathrm{SH}$ is formed early, during the cold collapse phase, when the CS accreted from the gas-phase is efficiently converted to $\mathrm{H}_{2} \mathrm{CS}$ via atomic hydrogen addition, and thence to $\mathrm{CH}_{3} \mathrm{SH}$. In common with the methanol-producing series, the addition of $\mathrm{H}$ to $\mathrm{CS}$ and $\mathrm{H}_{2} \mathrm{CS}$ is expected in both cases to have an activation energy barrier. Previous networks have assumed a value of $1000 \mathrm{~K}$ for $\mathrm{H}+\mathrm{CS} \rightarrow \mathrm{HCS}$ (e.g. Hasegawa et al. 1992), which is unaltered here. The hydrogenation of $\mathrm{H}_{2} \mathrm{CS}$ is a newly added process in this network, to which a barrier of $1000 \mathrm{~K}$ is also assigned. This reaction is allowed three product branches, each of which are given equal weight: $\mathrm{CH}_{3} \mathrm{~S}, \mathrm{CH}_{2} \mathrm{SH}$, and $\mathrm{HCS}+\mathrm{H}_{2}$ (corresponding to hydrogen abstraction). Most of the CS, that is accreted onto the grains during collapse, is ultimately converted into and stored as $\mathrm{CH}_{3} \mathrm{SH}$ in the ices.

Desorption of $\mathrm{CH}_{3} \mathrm{SH}$ from the dust grains becomes important above around $100 \mathrm{~K}$; peak gas-phase abundance for this molecule occurs at a temperature of $119 \mathrm{~K}$ (see Table 8). In spite of the identical binding energy adopted for methanethiol as for methanol, $\mathrm{CH}_{3} \mathrm{SH}$ still reaches a gas-phase peak at a somewhat lower temperature than $\mathrm{CH}_{3} \mathrm{OH}$; the large total abundance of methanol on the grains causes its release to take longer and peak later than for methanethiol. The peak gas-phase abundance of $\mathrm{CH}_{3} \mathrm{SH}$ is also lower than the peak value obtained on the grain surfaces; abstraction of hydrogen from methanethiol by surface radicals, including, most prominently, $\mathrm{NH}_{2}$, causes destruction of this molecule just prior to its release into the gas phase, reducing the overall peak by a factor of $\sim 3$. While similar $\mathrm{H}$-abstraction processes also exist for methanol, the exothermicity of these reactions is low and results in larger activation energy barriers (and thus smaller rates). Barriers for abstraction reactions are based on experimental values where available, or calculated using the Evans-Polanyi relation (as detailed by Garrod 2013); barriers involving methanethiol are obtained with the last method.

Formation of $\mathrm{C}_{2} \mathrm{H}_{5} \mathrm{SH}$ is achieved through three main processes, occurring at $\sim 20-30,45$, and $55 \mathrm{~K}$. The first is the addition reaction $\mathrm{CH}_{3}+\mathrm{CH}_{2} \mathrm{SH} \rightarrow \mathrm{C}_{2} \mathrm{H}_{5} \mathrm{SH}$; this process is analogous to the dominant mechanism for ethanol formation in the model, and $\mathrm{C}_{2} \mathrm{H}_{5} \mathrm{SH}$ abundance in the ices shows similar behavior to that of ethanol in the $20-40 \mathrm{~K}$ temperature range.
At around $45 \mathrm{~K}$, the addition of atomic $\mathrm{H}$ to the $\mathrm{C}_{2} \mathrm{H}_{5} \mathrm{~S}$ radical becomes more important, contributing an abundance of a few $10^{-10} \times n\left(\mathrm{H}_{2}\right)$. The $\mathrm{C}_{2} \mathrm{H}_{5} \mathrm{~S}$ radical is itself formed by the addition of $\mathrm{S}$ atoms to $\mathrm{C}_{2} \mathrm{H}_{5}$, which is produced when acetylene in the bulk ice mantles becomes mobile, allowing it to be hydrogenated on the ice surface.

At $55 \mathrm{~K}$, the main $\mathrm{C}_{2} \mathrm{H}_{5} \mathrm{SH}$-producing event occurs, when $\mathrm{H}_{2} \mathrm{~S}$ becomes mobile, dredges up from the bulk ice mantle, and desorbs from the ice surface. While most $\mathrm{H}_{2} \mathrm{~S}$ is desorbed into the gas phase, some reacts with atomic $\mathrm{H}$, which abstracts a hydrogen atom to give HS. This radical may then quickly react with $\mathrm{C}_{2} \mathrm{H}_{5}$ to produce ethanethiol. $\mathrm{C}_{2} \mathrm{H}_{5} \mathrm{SH}$ ultimately desorbs, reaching peak gas-phase abundance at a similar temperature to the alcohols. It should be noted that essentially all $\mathrm{H}_{2} \mathrm{~S}$ in the ices is converted to $\mathrm{C}_{2} \mathrm{H}_{5} \mathrm{SH}$ through this mechanism, as can be clearly seen in Fig. 3a. However, there is no analogous conversion of $\mathrm{H}_{2} \mathrm{~S}$ into $\mathrm{CH}_{3} \mathrm{SH}$, as the $\mathrm{CH}_{3}$ radical is no longer present in significant abundance when $\mathrm{H}_{2} \mathrm{~S}$ and $\mathrm{HS}$ become mobile on the grains. Neither does the addition of the $\mathrm{OH}$ radical to $\mathrm{CH}_{3} / \mathrm{C}_{2} \mathrm{H}_{5}$ provide any significant formation route for methanol/ethanol, analogous to the HS-related mechanisms. While the photodissociation of water ice provides abundant $\mathrm{OH}$, its mobility is relatively low at these temperatures.

By the end of the model run, at $400 \mathrm{~K}, \mathrm{CS}$ and $\mathrm{H}_{2} \mathrm{CS}$ comprise more than $50 \%$ of the sulfur budget; the remainder resides mostly in $\mathrm{SO}$ and $\mathrm{SO}_{2}$ (not shown).

\subsection{Discussion of modeling}

For the alcohols, the $\mathrm{CH}_{3} \mathrm{OH} / \mathrm{C}_{2} \mathrm{H}_{5} \mathrm{OH}$ ratio of 78 produced by the models, comparing peak abundances, is a reasonable match to the observed value of $\sim 20$. In spite of the approximate nature of the overall sulfur abundance in the models due to the uncertainty in initial atomic sulfur budget, the modeled $\mathrm{C}_{2} \mathrm{H}_{5} \mathrm{OH} / \mathrm{C}_{2} \mathrm{H}_{5} \mathrm{SH}$ ratio of 225 is in line with the observed lower limit of 125 .

Peak abundance for $\mathrm{CH}_{3} \mathrm{SH}$ produced by the model is somewhat lower than the observations demand. A modeled ratio of $\mathrm{CH}_{3} \mathrm{SH} / \mathrm{C}_{2} \mathrm{H}_{5} \mathrm{SH}=3.1$ is obtained, compared with the observed value of $>21$. Similarly, the ratio of methanol to methanethiol is around 50 times larger ( $\sim 5700$ versus 120$)$ than the observed ratio, suggesting that the low $\mathrm{CH}_{3} \mathrm{SH} / \mathrm{C}_{2} \mathrm{H}_{5} \mathrm{SH}$ ratio is caused more by an underprediction of $\mathrm{CH}_{3} \mathrm{SH}$ relative to the other three molecules rather than by an overprediction of $\mathrm{C}_{2} \mathrm{H}_{5} \mathrm{SH}$. However, it should be noted that $\mathrm{CH}_{3} \mathrm{SH}$ is released from the grains at lower temperatures than either methanol or ethanethiol in the models. This would tend to produce a somewhat larger spatial extent for methanethiol under a three-dimensional treatment of the core, and would likely result in a greater average column density than is indicated when comparing peak abundances for each molecule. The differing sublimation characteristics of $\mathrm{CH}_{3} \mathrm{SH}$ may therefore improve its column density ratio with other molecules. Direct laboratory determination of the methanethiol binding energy on a water/methanol surface would give greater confidence to the comparison with observed column density values.

The low initial abundance of sulfur assumed in this model allows room for the methanethiol abundance to be increased through this variable; however, alternative model runs indicate that ethanethiol abundances also scale in this way. Lower efficiency in the early-stage formation of $\mathrm{H}_{2} \mathrm{~S}$ on the grains could reduce the $\mathrm{C}_{2} \mathrm{H}_{5} \mathrm{SH}$ abundance, as could lower acetylene production. Acetylene abundances ultimately rely on the quantity of ice-mantle methane formed during the cold collapse stage, 

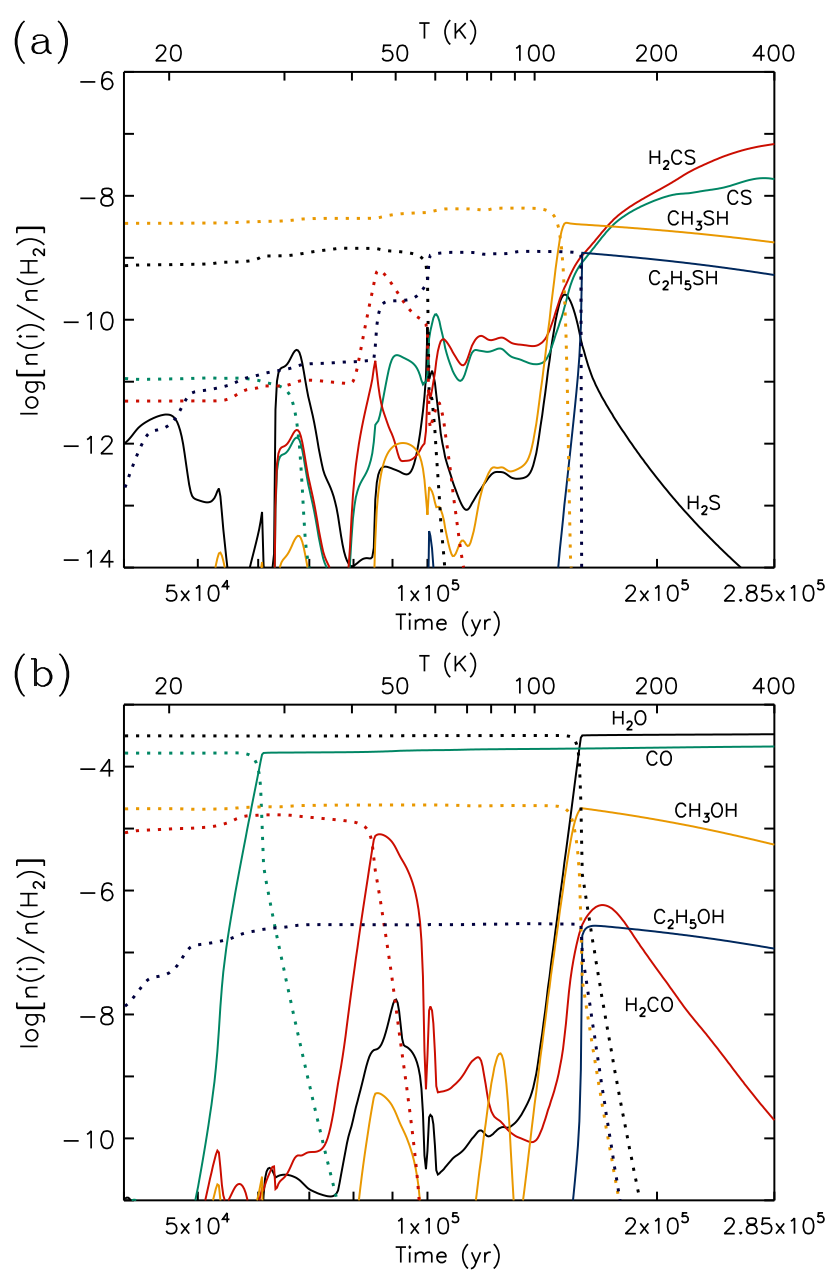

Fig. 3. Chemical model abundances for a selection of sulfur-bearing molecules a) and their oxygen-bearing analogs b). Solid lines indicate gas-phase abundances; dotted lines of the same color indicate the grainsurface/ice abundances of the same species.

Table 8. Results of chemical kinetics modeling of alkanethiols and alkanols toward Sgr B2(N2).

\begin{tabular}{lcc}
\hline \hline Species & Peak $n[\mathrm{i}] / n\left(\mathrm{H}_{2}\right)$ & Temperature $(\mathrm{K})$ at peak \\
\hline $\mathrm{CH}_{3} \mathrm{SH}$ & $3.7 \times 10^{-9}$ & 119 \\
$\mathrm{C}_{2} \mathrm{H}_{5} \mathrm{SH}$ & $1.2 \times 10^{-9}$ & 130 \\
$\mathrm{CH}_{3} \mathrm{OH}$ & $2.1 \times 10^{-5}$ & 130 \\
$\mathrm{C}_{2} \mathrm{H}_{5} \mathrm{OH}$ & $2.7 \times 10^{-7}$ & 139 \\
\hline
\end{tabular}

which is released into the gas phase at temperatures around $25 \mathrm{~K}$, producing a range of hydrocarbons.

\section{Discussion}

In light of the extensive number of emission features detected for $\mathrm{CH}_{3} \mathrm{OH}, \mathrm{C}_{2} \mathrm{H}_{5} \mathrm{OH}$, and $\mathrm{CH}_{3} \mathrm{SH}$ in our sensitive ALMA data toward Sgr B2(N2), it is noteworthy that we detect only a few, rather weak, and partially or completely blended lines that can be attributed to $\mathrm{C}_{2} \mathrm{H}_{5} \mathrm{SH}$. Consequently, we are unable to claim even a tentative detection of the molecule. We consider the low upper limit to the column density of ethanethiol (Table 7) to be meaningful. In fact, comparing the Sgr B2(N2) column density ratios of alkanols and alkanethiols (or their lower limits) in
Table 9. Column density ratios of alkanols and alkanethiols in different sources.

\begin{tabular}{|c|c|c|c|c|}
\hline Source & $\begin{array}{c}\mathrm{MeOH} \\
\mathrm{EtOH}\end{array}$ & $\begin{array}{c}\mathrm{MeSH} \\
\mathrm{EtSH}\end{array}$ & $\begin{array}{l}\mathrm{MeOH} \\
\mathrm{MeSH}\end{array}$ & $\begin{array}{l}\mathrm{EtOH} \\
\mathrm{EtSH} \\
\end{array}$ \\
\hline Sgr B2(N2) & 20 & $\gtrsim 21$ & 118 & $\gtrsim 125$ \\
\hline Orion $\mathrm{KL}^{a, b}$ & 31 & $5^{c}$ & 120 & $20^{c}$ \\
\hline G327.3-0.6 $6^{a, d}$ & 82 & & 58 & \\
\hline
\end{tabular}

Notes. ${ }^{(a)}$ Methanol $(\mathrm{MeOH})$ inferred from ${ }^{13} \mathrm{CH}_{3} \mathrm{OH}$. EtOH, MeSH, and EtSH short for ethanol, methanethiol, and ethanethiol, respectively. (b) Orion KL data from Kolesniková et al. (2014) . ${ }^{(c)}$ Only for gaucheEtSH; ratio would be smaller by about a factor of 1.25 with anti-EtSH assuming LTE. ${ }^{(d)}$ G327.3-0.6 data from Gibb et al. (2000).

Table 9, it is conceivable that the column density of $\mathrm{C}_{2} \mathrm{H}_{5} \mathrm{SH}$ is close to the upper limit. With so many potential ethanethiol lines partially or strongly blended in our ALMA data, additional observations at longer wavelengths may be more promising than more sensitive observations at $3 \mathrm{~mm}$ or observations at shorter wavelengths.

The non-detection of ethanethiol in Sgr B2(N2) is rather surprising in the context of the purported detection of the molecule in Orion $\mathrm{KL}$ at a column density that yields $\mathrm{CH}_{3} \mathrm{SH} / \mathrm{C}_{2} \mathrm{H}_{5} \mathrm{SH}$ and $\mathrm{C}_{2} \mathrm{H}_{5} \mathrm{OH} / \mathrm{C}_{2} \mathrm{H}_{5} \mathrm{SH}$ ratios that are much lower than our lower limits. The ratios (Table 9) are 5 and 20, respectively, ignoring the presence of the anti-conformer, or 4 and 16 if we assume a thermal $(200 \mathrm{~K})$ population of this conformer. Our numbers are $\gtrsim 21$ and $\gtrsim 125$, respectively. In contrast, the $\mathrm{CH}_{3} \mathrm{OH} / \mathrm{CH}_{3} \mathrm{SH}$ ratios are identical, and the $\mathrm{CH}_{3} \mathrm{OH} / \mathrm{C}_{2} \mathrm{H}_{5} \mathrm{OH}$ values are quite similar, 20 and 31, respectively. Inspection of the features reported in Kolesniková et al. (2014) as detected shows one isolated emission feature (near $243.58 \mathrm{GHz}$ ) assigned to ethanethiol. A small number of fairly isolated lines were assigned in part to the molecule. The majority of the potential $\mathrm{C}_{2} \mathrm{H}_{5} \mathrm{SH}$ lines, however, are strongly blended. More importantly, the model of all species excluding $\mathrm{C}_{2} \mathrm{H}_{5} \mathrm{SH}$ leaves no room for any ethanethiol contribution in the isolated emission feature near $263.90 \mathrm{GHz}$. The high abundance of $\mathrm{C}_{2} \mathrm{H}_{5} \mathrm{SH}$ relative to $\mathrm{CH}_{3} \mathrm{SH}$ and the alkanols in Orion KL compared to $\mathrm{Sgr} \mathrm{B} 2(\mathrm{~N} 2)$, and the issues we pointed out concerning the identification of $\mathrm{C}_{2} \mathrm{H}_{5} \mathrm{SH}$ in the Orion $\mathrm{KL}$ spectrum suggest that the presence of ethanethiol in Orion $\mathrm{KL}$ is uncertain. Unfortunately, our chemical model calculations are not accurate enough, in particular for $\mathrm{CH}_{3} \mathrm{SH}$, to support or dismiss the reported $\mathrm{C}_{2} \mathrm{H}_{5} \mathrm{SH}$ detection in Orion KL.

The column density of $\mathrm{CH}_{3} \mathrm{SH}$ is relatively high in G327.3-0.6, yielding a $\mathrm{CH}_{3} \mathrm{OH} / \mathrm{CH}_{3} \mathrm{SH}$ ratio of 58 (Gibb et al. 2000), half the value in Sgr B2(N2) and Orion KL. On the other hand, the $\mathrm{CH}_{3} \mathrm{OH} / \mathrm{C}_{2} \mathrm{H}_{5} \mathrm{OH}$ ratio is 82 , higher than in Orion KL, and even higher than in Sgr B2(N2). On the basis of column density ratios alone, it is difficult to judge how promising a search for ethanethiol in G327.3-0.6 would be. We point out that we did not use the quoted column density of $\mathrm{CH}_{3} \mathrm{OH}$ from Gibb et al. (2000). Instead, we used the ${ }^{13} \mathrm{CH}_{3} \mathrm{OH}$ column density and the quoted ${ }^{12} \mathrm{C} /{ }^{13} \mathrm{C}$ ratio of 40 ; the reported $\mathrm{CH}_{3} \mathrm{OH} /{ }^{13} \mathrm{CH}_{3} \mathrm{OH}$ ratio was actually 10 , suggesting substantial $\mathrm{CH}_{3} \mathrm{OH}$ opacities.

In their $1.3 \mathrm{~mm}$ line survey toward three positions in Sgr B2, Nummelin et al. $(1998,2000)$ detected $\mathrm{CH}_{3} \mathrm{OH}, \mathrm{C}_{2} \mathrm{H}_{5} \mathrm{OH}$, and $\mathrm{CH}_{3} \mathrm{SH}$ toward not only Sgr B2(N), but also toward Sgr B2(M). Judging from their ${ }^{12} \mathrm{C} /{ }^{13} \mathrm{C}$ ratios for methanol, these column densities are affected by substantial opacities. Emission of $\mathrm{C}_{2} \mathrm{H}_{5} \mathrm{OH}$ and $\mathrm{CH}_{3} \mathrm{SH}$ may be less prone to opacity biases. Their 
ratios are 3.8 and 1.4 for Sgr $\mathrm{B} 2(\mathrm{~N})$, whose emission is likely dominated by Sgr B2(N1), and Sgr B2(M), respectively. Both values are smaller than 5.9 for Sgr B2(N2), though not by much for Sgr B2(N). The Orion KL value is essentially identical to the one from Sgr B2(N), whereas the G327.3-0.6 value of 0.7 is even more extreme than the one from Sgr B2(M).

Our upper limit to the column density of normal-propanol is not very constraining in comparison to our $\mathrm{CH}_{3} \mathrm{OH} / \mathrm{C}_{2} \mathrm{H}_{5} \mathrm{OH}$ ratio of 20 , as it translates to a $\mathrm{C}_{2} \mathrm{H}_{5} \mathrm{OH} / n-\mathrm{C}_{3} \mathrm{H}_{7} \mathrm{OH}$ ratio of $\gtrsim 7.7$. In the case of the Galactic center molecular cloud MC $\mathrm{G}+0.693-0.027$, the column density ratio between $\mathrm{CH}_{3} \mathrm{OH}$ and $\mathrm{C}_{2} \mathrm{H}_{5} \mathrm{OH}$ is $\sim 14$ (Requena-Torres et al. 2008), lower than the Sgr B2(N2) value, but the lower limit to the $\mathrm{C}_{2} \mathrm{H}_{5} \mathrm{OH}$ to $n$ $\mathrm{C}_{3} \mathrm{H}_{7} \mathrm{OH}$ ratio is only $\sim 4.5$, which was derived from the $\mathrm{CH}_{3} \mathrm{OH}$ to $\mathrm{C}_{2} \mathrm{H}_{5} \mathrm{OH}$ and the $\mathrm{CH}_{3} \mathrm{OH}$ to $n-\mathrm{C}_{3} \mathrm{H}_{7} \mathrm{OH}$ ratios (RequenaTorres et al. 2008). In their paper on the tentative detection of ethyl methyl ether, Tercero et al. (2015) mention a ground state column density ratio of $\gtrsim 60$. Taking column density corrections by vibrational states for both molecules and by other conformers for $n$-propanol into account (see Table 7) the column density ratio becomes $\gtrsim 14.3$, slightly more constraining than ours. However, should our $\mathrm{C}_{2} \mathrm{H}_{5} \mathrm{CN} / n-\mathrm{C}_{3} \mathrm{H}_{7} \mathrm{CN}$ ratio of $\sim 55$ (Belloche et al. 2016 and rescaled for the same source size of $1.2^{\prime \prime}$ in the case of $n-\mathrm{C}_{3} \mathrm{H}_{7} \mathrm{CN}$, Belloche et al. 2014) be more appropriate for the estimation of the column density of $n-\mathrm{C}_{3} \mathrm{H}_{7} \mathrm{OH}$, then all available observational results are not so meaningful. Our upper limit to the column density of iso-propanol translates to a $\mathrm{C}_{2} \mathrm{H}_{5} \mathrm{OH} / i-\mathrm{C}_{3} \mathrm{H}_{7} \mathrm{OH}$ ratio of $\gtrsim 21.5$, which may be meaningful if the $i-\mathrm{C}_{3} \mathrm{H}_{7} \mathrm{OH} / n-\mathrm{C}_{3} \mathrm{H}_{7} \mathrm{OH}$ ratio is considerably larger than the $i-\mathrm{C}_{3} \mathrm{H}_{7} \mathrm{CN} / n-\mathrm{C}_{3} \mathrm{H}_{7} \mathrm{CN}$ ratio of $\sim 0.4$ in Sgr B2(N2) (Belloche et al. 2014).

Our tentative detection of torsionally excited methanethiol is the first such account; previously only ground state transitions had been identified. On the other hand, observations of $\mathrm{CH}_{3} \mathrm{OH}$, $v_{\mathrm{t}}=2$ and ${ }^{13} \mathrm{CH}_{3} \mathrm{OH}, v_{\mathrm{t}}=1$ had been reported before, e.g., in Sgr B2(N) by Nummelin et al. (1998) and in Orion KL by Schilke et al. (2001).

We determined an isotopic ratio of $\sim 25$ for $\mathrm{CH}_{3} \mathrm{OH} /{ }^{13} \mathrm{CH}_{3} \mathrm{OH}$ and for the ratio of parent ethanol to both of its isotopologs with one ${ }^{13} \mathrm{C}$. This value is slightly larger than our ratio determined for the main isotopic species of vinyl cyanide to all three of its singly ${ }^{13} \mathrm{C}$ substituted species of $21 \pm 1$ (Müller et al. 2008). These ratios are in good to reasonable agreement with a value of $\sim 20$ in Galactic center sources (Wilson \& Rood 1994; Milam et al. 2005) or the range of $\sim 20$ to 25 derived by Güsten et al. (1985).

Our $\mathrm{CH}_{3} \mathrm{OH} / \mathrm{CH}_{3}^{18} \mathrm{OH}$ and ${ }^{13} \mathrm{CH}_{3} \mathrm{OH} / \mathrm{CH}_{3}^{18} \mathrm{OH}$ ratios of $\sim 180$ and $\sim 7.3$ are in very good agreement with respective Sgr B2 values of $210 \pm 40$ and $7.5 \pm 1.0$ (Gardner et al. 1989) or with $\sim 200$ and $8.6 \pm 1.2$ for respective $\mathrm{H}_{2} \mathrm{CO}$ values in Sgr B2 and the Galactic center source M-0.13-0.18 (Güsten et al. 1985). Wilson \& Rood (1994) summarized isotopic ratios of $\sim 250$ and $\sim 12.5$ in the Galactic center ISM, in reasonable agreement with our values.

The isotopic ratios in the Galactic center ISM are rather different from the solar system values of about 89 and 490 for the ${ }^{12} \mathrm{C} /{ }^{13} \mathrm{C}$ and ${ }^{16} \mathrm{O} /{ }^{18} \mathrm{O}$ ratios, respectively. These changes reflect gradients in the isotopic ratios from the center of the Milky Way to its outer regions which, in addition, change with time. This aspect explains small, but non-negligible differences between the solar system values and those determined in the local ISM (e.g., Güsten et al. 1985; Wilson \& Rood 1994; Milam et al. 2005).

\section{Conclusion}

Our sensitive ALMA data permitted clear detections of methanethiol with the first, albeit tentative, detection of lines in its first excited torsional state, ethanol, with the first unambiguous detection of the singly ${ }^{13} \mathrm{C}$ substituted isotopomers, and three isotopologs of methanol; the main species was detected in several torsional states, including some evidence of its third excited torsional state. However, they were not sensitive enough for a clear detection of ethanethiol, though a comparison to $\mathrm{CH}_{3} \mathrm{SH}$ and alkanols suggests that its column density could be close to the upper limit derived here. We also obtained upper limits to the column densities of normal- and iso-propanol. Our values for the ${ }^{12} \mathrm{C} /{ }^{13} \mathrm{C}$ ratio obtained for methanol and ethanol and for the ${ }^{16} \mathrm{O} /{ }^{18} \mathrm{O}$ ratio obtained for methanol are in line with the values known for the ISM in the Galactic center region.

The column density ratios involving methanol, ethanol, and methanethiol in Sgr B2(N2) are similar to values reported for Orion KL, but those involving ethanethiol are significantly different and suggest that the detection of ethanethiol reported toward Orion KL is rather uncertain.

The chemical models presented here include new reactions for the formation and destruction of methanethiol and ethanethiol. This represents a step forward in the complexity of sulfur chemistry that can be treated with astrochemical models. The results indicate that adequate abundances of both alkanethiols may be formed in hot cores through dust-grain surface/icemantle chemistry. Methanethiol is formed at the low temperatures achieved during cold core collapse (on the order $10 \mathrm{~K}$ ). Ethanethiol is produced on the grains at elevated temperatures ( 45-55 K), with most of the surface $\mathrm{H}_{2} \mathrm{~S}$ at those temperatures being converted almost entirely to ethanethiol. The modeled gas-phase ratio $\mathrm{CH}_{3} \mathrm{SH} / \mathrm{C}_{2} \mathrm{H}_{5} \mathrm{SH} \simeq 3$ is substantially lower than the observed value of $>21$. The strongest influence on this ratio is likely the underprediction of $\mathrm{CH}_{3} \mathrm{SH}$ (in comparison to $\mathrm{CH}_{3} \mathrm{OH}$ ), although the efficient formation of $\mathrm{C}_{2} \mathrm{H}_{5} \mathrm{SH}$ from $\mathrm{H}_{2} \mathrm{~S}$ on the grains is also a source of uncertainty.

In addition, more sensitive observations of $\mathrm{Sgr} \mathrm{B} 2(\mathrm{~N})$, Orion KL, or other sources with ALMA or other interferometers are required to establish whether and possibly in what quantities ethanethiol, normal-propanol, and iso-propanol are present in the ISM. Such observations may be more promising at wavelengths longer than $3 \mathrm{~mm}$ for the first two molecules.

Acknowledgements. This paper makes use of the following ALMA data: ADS/JAO.ALMA\#2011.0.00017.S, ADS/JAO.ALMA\#2012.1.00012.S. ALMA is a partnership of ESO (representing its member states), NSF (USA) and NINS (Japan), together with NRC (Canada), NSC and ASIAA (Taiwan), and KASI (Republic of Korea), in cooperation with the Republic of Chile. The Joint ALMA Observatory is operated by ESO, AUI/NRAO and NAOJ. The interferometric data are available in the ALMA archive at https://almascience.eso.org/ $\mathrm{aq} /$. This work has been supported by the Deutsche Forschungsgemeinschaft (DFG) through the collaborative research grant SFB 956 "Conditions and Impact of Star Formation", project area B3. We thank Dr. Monika Koerber for making an ethanol spectrum available for inspection of the relative intensities. L.H.X. and R.M.L. acknowledge financial support from the Natural Sciences and Engineering Research Council (NSERC) of Canada. R.T.G. acknowledges support from the NASA Astrophysics Theory Program through grant NNX11AC38G. A.W. acknowledges financial support from the CNRS program "Physique et Chimie du Milieu Interstellaire (PCMI). Our research benefited from NASA's Astrophysics Data System (ADS).

\section{References}

Anderson, D. E., Bergin, E. A., Maret, S., Wakelam, V. 2013, ApJ, 779, 141 Ball, J. A., Gottlieb, C. A., Lilley, A. E., \& Radford, H. E. 1970, ApJ, 162, L203 Belloche, A., Menten, K. M., Comito, C., et al. 2008, A\&A, 482, 179 Belloche, A., Garrod, R. T., Müller, H. S. P., et al. 2009, A\&A, 499, 215 
Belloche, A., Müller, H. S. P., Menten, K. M., Schilke, P., \& Comito, C. 2013, A\&A, 559, A47

Belloche, A., Garrod, R. T., Müller, H. S. P., \& Menten, K. M. 2014, Science, 345,1584

Belloche, A., Müller, H. S. P., Garrod, R. T., \& Menten, K. M. 2016, A\&A, 587, A91

Bettens, F. L., Sastry, K. V. L. N., Herbst, E., et al. 1999, ApJ, 510, 789

Bisschop, S. E., Jørgensen, J. K., van Dishoeck, E. F., \& de Wachter, E. B. M. 2007, A\&A, 465, 913

Bisschop, S. E., Jørgensen, J. K., Bourke, T. L., Bottinelli, S., \& van Dishoeck, E. F. 2008, A\&A, 488, 959

Bouchez, A., Walters, A., Müller, H. S. P., et al. 2012, J. Quant. Spectr. Rad. Transf., 113, 1148

Cernicharo, J., Marcelino, N., Roueff, E., et al. 2012, ApJ, 759, L43

Christen, D., \& Müller, H. S. P. 2003, Phys. Chem. Chem. Phys., 5, 3600

Cohen, E. A., \& Pickett, H. M. 1982, J. Mol. Spectr., 93, 83

Collings, M. P., Anderson, M. A., Chen, R., et al. 2004, MNRAS, 354, 1133

Coudert, L. H., Drouin, B. J., Tercero, B., et al. 2013, ApJ, 779, 119

Crockett, N. R., Bergin, E. A., Neill, J. L., et al. 2014, ApJ, 787, 112

Dobrowolski, J. C., Ostrowski, S., Kołos, R., \& Jamróz, M. H. 2008, Vib. Spectr., 48, 82

Durig, J. R., \& Larsen, R. A. 1990, J. Mol. Struct, 238, 195

Durig, J. R., Bucy, W. E., Wurrey, C. J., \& Carreira, L. A. 1975, J. Phys. Chem., 79,988

Endres, C. P., Müller, H. S. P., Brünken, S., et al. 2006, J. Mol. Struct, 795, 242

Endres, C. P., Drouin, B. J., Pearson, J. C., et al. 2009, A\&A, 504, 635

Fisher, J., Paciga, G., Xu, L.-H., et al. 2007, J. Mol. Spectr., 245, 7

Fortman, S. M., Neese, C. F., \& De Lucia, F. C. 2014, ApJ, 782, 75

Fukushima, K., \& Zwolinski, B. J. 1968, J. Mol. Spectr., 26, 368

Gardner, F. F., \& Whiteoak, J. B. 1981, MNRAS, 194, 37P

Gardner, F. F., Whiteoak, J. B., Reynolds, J., Peters, W. L., \& Kuiper, T. B. H 1989, MNRAS, 240, 35P

Garrod, R. T. 2013, ApJ, 765, 60

Garrod, R. T., Widicus Weaver, S. L., Herbst, E., 2008, ApJ, 682, 283

Gottlieb, C. A., Ball, J. A., Gottlieb, E. W., \& Dickinson, D. F. 1979, ApJ, 227, 422

Gibb, E., Nummelin, A., Irvine, W. M., Whittet, D. C. B., \& Bergman, P. 2000, ApJ, 545, 309

Güsten, R., Henkel, C., \& Batrla, W. 1985, A\&A, 149, 195

Henkel, C., Wilson, T. L., Walmsley, C. M., \& Pauls, T. 1983, A\&A, 127, 388

Hasegawa, T. I., Herbst, E. \& Leung, C. M. 1992, ApJS, 82, 167

Herbst, E., Messer, J. K., De Lucia, F. C., \& Helminger, P. 1984, J. Mol. Spectr., 108,42

Hirota, E. 1979, J. Phys. Chem., 83, 1457

Hollis, J. M., Lovas, F. J., Jewell, P. R., \& Coudert, L. H. 2002, ApJ, 571, L59

Hoshino, Y., Ohishi, M., Akabane, K., et al. 1996, ApJS, 104, 317

Ikeda, M., Duan, Y.-B., Tsunekawa, S., \& Takagi, K. 1998, ApJS, 117, 249

Ikeda, M., Ohishi, M., Nummelin, A., et al. 2001, ApJ, 560, 792

Inagaki, F., Harada, I., \& Shimanouchi, T. 1973, J. Mol. Spectr., 46, 381

Ivash, E. V., \& Dennison, D. M. 1953, J. Chem. Phys., 21, 1804

Jørgensen, J. K., Favre, C., Bisschop, S. E., et al. 2012, ApJ, 757, L4

Kakar, R. K., \& Quade, C. R. 1980, J. Chem. Phys., 72, 4300

Kahn, K., \& Bruice, T. C. 2005, Chem. Phys. Chem., 6, 487

Kisiel, Z., Dorosh, O., Maeda, A., et al. 2010, Phys. Chem. Chem. Phys., 12, 8329

Kleiner, I. 2010, J. Mol. Spectr., 260, 1

Koerber, M., Bisschop, S. E., Endres, C. P., et al. 2013, A\&A, 558, A112

Kolesniková, L., Daly, A. M., Alonso, J. L., Tercero, B., \& Cernicharo, J. 2013, J. Mol. Spectr., 289, 13

Kolesniková, L., Tercero, B., Cernicharo, J., et al. 2014, ApJ, 784, L7

Kuriyama, H., Takagi, K., Takeo, H., \& Matsumura, C. 1986, ApJ, 311, 1073

Lees, R. M., \& Baker, J. G. 1968, J. Chem. Phys., 48, 5299

Lees, R. M., \& Mohammadi, M. A. 1980, Can. J. Phys., 58, 1640

Leurini, S., Schilke, P., Menten, K. M., et al. 2004, A\&A, 422, 573

Leurini, S., Schilke, P., Wyrowski, F., \& Menten, K. M. 2007, A\&A, 466, 215

Linke, R. A., Frerking, M. A., \& Thaddeus, P. 1979, ApJ, 234, L139

Loomis, R. A., Zaleski, D. P., Steber, A. L., et al. 2013, ApJ, 765, L9

Maeda, A., De Lucia, F. C., Herbst, E., et al. 2006a, ApJS, 162, 428

Maeda, A., Medvedev, I. R., De Lucia, F. C., \& Herbst, E. 2006b, ApJS, 166 650

Manocha, A. S., Fateley, W. G., \& Shimanouchi, T. 1973, J. Phys. Chem., 77, 1977

Maury, A. J., Belloche, A., André, P., et al. 2014, A\&A, 563, L2

McMillan, J. P., Fortman, S. M., Neese, C. F., \& De Lucia, F. C. 2014, ApJ, 795, 56

McNaughton, D., \& Evans, C. J. 1999, J. Mol. Spectr., 196, 274
Milam, S. N., Savage, C., Brewster, M. A., Ziurys, L. M., \& Wyckoff, S. 2005, ApJ, 634, 1126

Moruzzi, G., Winnewisser, B. P., Winnewisser, M., Mukhopadhyay, I., \& Strumia, F. 1995, Microwave, infrared \& laser transitions of methanol: Atlas of assigned lines from 0 to $1258 \mathrm{~cm}^{-1}$ (Boca Raton, FL, USA: CRC Press)

Müller, H. S. P. 2013, J. Quant. Spectr. Rad. Transf., 130, 335

Müller, H. S. P., Thorwirth, S., Roth, D. A., \& Winnewisser, G. 2001, A\&A, 370, L49

Müller, H. S. P., Menten, K. M., Mäder, H. 2004, A\&A, 428, 1019

Müller, H. S. P., Schlöder, F., Stutzki, J., \& Winnewisser, G. 2005, J. Mol. Struct, 742,215

Müller, H. S. P., Belloche, A., Menten, K. M., et al. 2008, J. Mol. Spectr., 251, 319

Müller, H. S. P., Dong, F., Nesbitt, D. J., Furuya, T., \& Saito, S. 2010, Phys. Chem. Chem. Phys., 12, 8362

Mukhopadhyay, I., \& Sastry, K. V. L. N. 2015, J. Mol. Spectr., 312, 51

Neill, J. L., Crockett, N. R., Bergin, E. A., Pearson, J. C., \& Xu, L.-H. 2013, ApJ, 777, 85

Neill, J. L., Bergin, E. A., Lis, D. C., et al. 2014, ApJ, 789, 8

Nummelin, A., Bergman, P., Hjalmarson, Å., et al. 1998, ApJS, 117, 427

Nummelin, A., Bergman, P., Hjalmarson, Å., et al. 2000, ApJS, 128, 213

Pearson, J. C., \& Drouin, B. J. 2005, J. Mol. Spectr., 234, 149

Pearson, J. C., Sastry, K. V. L. N., Herbst, E., \& De Lucia, F. C. 1996, J. Mol. Spectr., 175, 246

Pearson, J. C., Sastry, K. V. L. N., Herbst, E., \& De Lucia, F. C. 1997, ApJ, 480 420

Pearson, J. C., Brauer, C. S., \& Drouin, B. J. 2008, J. Mol. Spectr., 251, 394

Pearson, J. C., Brauer, C. S., Drouin, B. J., \& Xu, L.-H. 2009, Can. J. Phys., 87, 449

Pearson, J. C., Daly, A. M., \& Lees, R. M. 2015, J. Mol. Spectr., 318, 70

Pickett, H. M. 1972, J. Chem. Phys., 56, 1715

Pickett, H. M. 1991, J. Mol. Spectr., 148, 371

Pickett, H. M., Poynter, R. L., Cohen, E. A., et al. 1998, J. Quant. Spectr. Rad. Transf., 60, 883

Puzzarini, C., Senent, M. L., Domínguez-Gómez, R., et al. 2014, ApJ, 796, 50

Qin, S.-L., Schilke, P., Rolffs, R., et al. 2011, A\&A, 530, L9

Requena-Torres, M. A., Martín-Pintado, J., Rodríguez-Franco, A., et al. 2006 , A\&A, 455, 971

Requena-Torres, M. A., Martín-Pintado, J., Martín, S., \& Morris, M. R. 2008, ApJ, 672, 352

Sastry, K. V. L. N., Lees, R. M., \& De Lucia, F. C. 1984, J. Mol. Spectr., 103, 486

Sastry, K. V. L. N., Herbst, E., Booker, R. A., \& De Lucia, F. C. 1986, J. Mol. Spectr., 116, 120

Sastry, K. V. L. N., Vanderlinde, J., Donovan, D., Mukhopadhyay, I., \& Gupta, P. K. 1994, J. Mol. Spectr., 168, 374

Sastry, K. V. L. N., Mukhopadhyay, I., Gupta, P. K., \& VanderLinde, J. 1996, J. Mol. Spectr., 176, 38

Schilke, P., Benford, D. J., Hunter, T. R., Lis, D. C., \& Phillips, T. G. 2001, ApJS, 132,281

Schmidt, R. E., \& Quade, C. R. 1975, J. Chem. Phys., 62, 3864

Smirnov, I. A., Alekseev, E. A., Piddyachiy, V. I., Ilyushin, V. V., \& Motiyenko, R. A. 2013, J. Mol. Spectr., 293, 33

Smith, D., Devlin, J. P., \& Scott, D. W. 1968, J. Mol. Spectr., 25, 174

Snyder, L. E., Kuan, Y.-J., \& Miao, Y. 1994, Lect. Notes Phys., 439, 187

Suenram, R. D., Lovas, F. J., \& Pickett, H. M. 1986a, J. Mol. Spectr., 116, 406

Suenram, R. D., Lovas, F. J., \& Pickett, H. M. 1986b,J. Mol. Spectr., 119, 446

Takano, M., Sasada, Y., \& Satoh, T. 1968, J. Mol. Spectr., 26, 157

Takano, S., Sakai, Y., Kakimoto, S., Sasaki, M., \& Kobayashi, K. 2012, PASJ, 64, 89

Tercero, B., Kleiner, I., Cernicharo, J., et al. 2013, ApJ, 770, L13

Tercero, B., Cernicharo, J., López, A., et al. 2015, A\&A, 582, L1

Tsunekawa, S., Taniguchi, I., Tambo, A., et al. 1989, J. Mol. Spectr., 134, 63

Tsunekawa, S., Ukai, T. Toyama, A., \& Takagi, K. 1995, Microwave frequencies of the $\mathrm{CH}_{3} \mathrm{OH}$ molecule in the frequency range 7 to $200 \mathrm{GHz}$, unpublished report, Dep. of Physics, Toyama University, Toyama, Japan

Turner, B. E. 1977, ApJ, 213, L75

Wang, S., Bergin, E. A., Crockett, N. R., et al. 2011, A\&A, 527, A95

Wilson, T. L., \& Rood, R. 1994, ARA\&A, 32, 191

Xu, L.-H., \& Lovas, F. J. 1997, J. Phys. Chem. Ref. Data, 26, 17

Xu, L.-H., Walsh, M. S., \& Lees, R. M. 1996, J. Mol. Spectr., 179, 269

Xu, L.-H., Fisher, J., Lees, R. M., et al. 2008, J. Mol. Spectr., 251, 305

Xu, L.-H., Lees, R. M., Crabbe, G. T., et al. 2012, J. Chem. Phys., 137, 104313

Xu, L.-H., Lees, R. M., Hao, Y., et al. 2014, J. Mol. Spectr., 303, 1

Zaleski, D. P., Seifert, N. A., Steber, A. L., et al. 2013, ApJ, 765, L10

Zuckerman, B., Turner, B. E., Johnson, D. R., et al. 1975, ApJ, 196, L99 


\section{Appendix A: Additional figures}
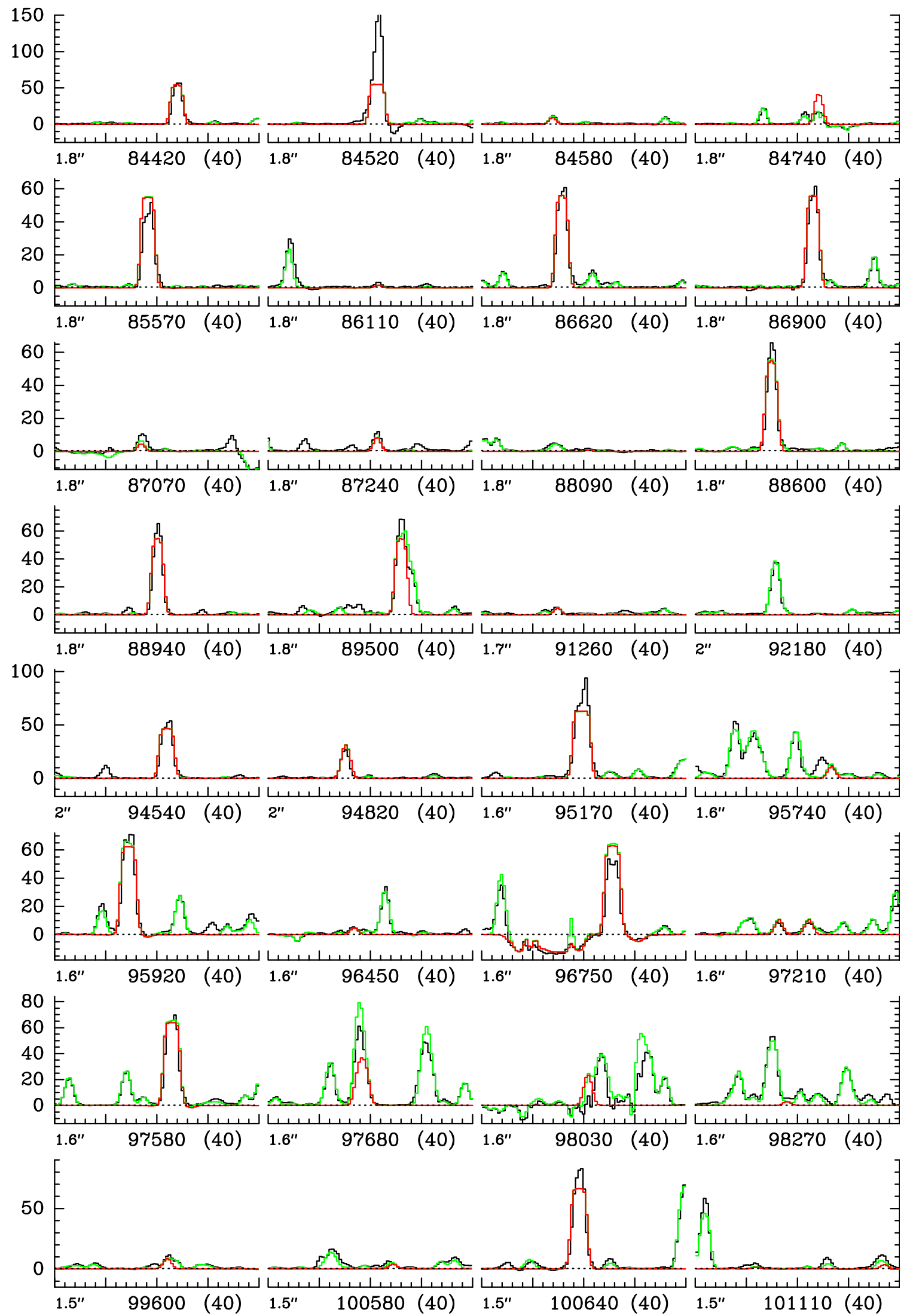

Fig. A.1. Transitions of $\mathrm{CH}_{3} \mathrm{OH}, v=0$ covered by our ALMA survey. The best-fit LTE synthetic spectrum of $\mathrm{CH}_{3} \mathrm{OH}$ is displayed in red and overlaid on the observed spectrum of Sgr B2(N2) shown in black. The green synthetic spectrum contains the contributions of all molecules identified in our survey so far including the species shown in red. The central frequency of each panel is indicated below the $x$-axis in MHz; its width is given also in MHz in parentheses. The angular resolution is also indicated. The $y$-axis is labeled in brightness temperature units (K). The dotted line marks the $3 \sigma$ noise level. 

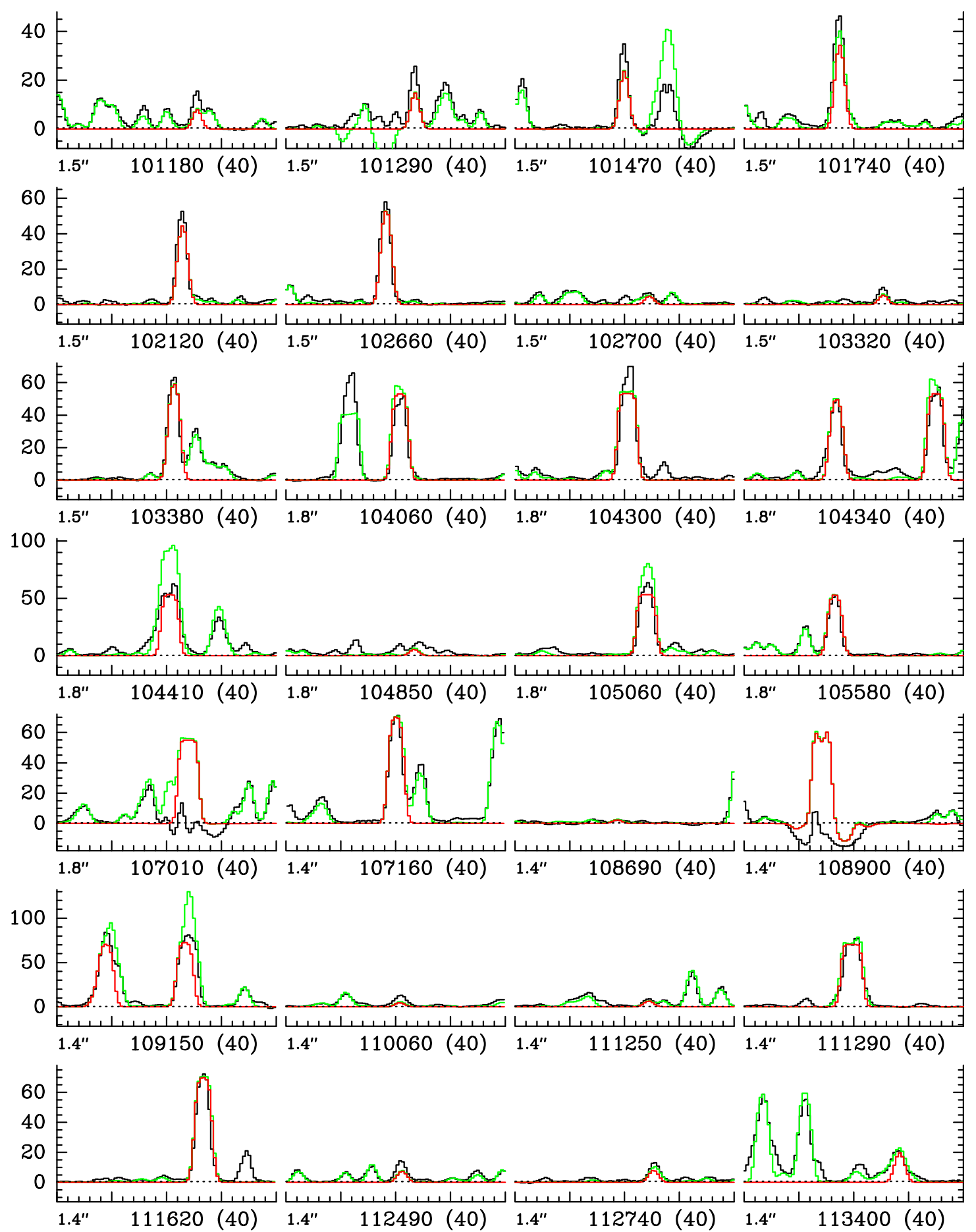

Fig. A.1. continued. 
H. S. P. Müller et al.: Alkanethiols and alkanols in Sgr B2(N2)
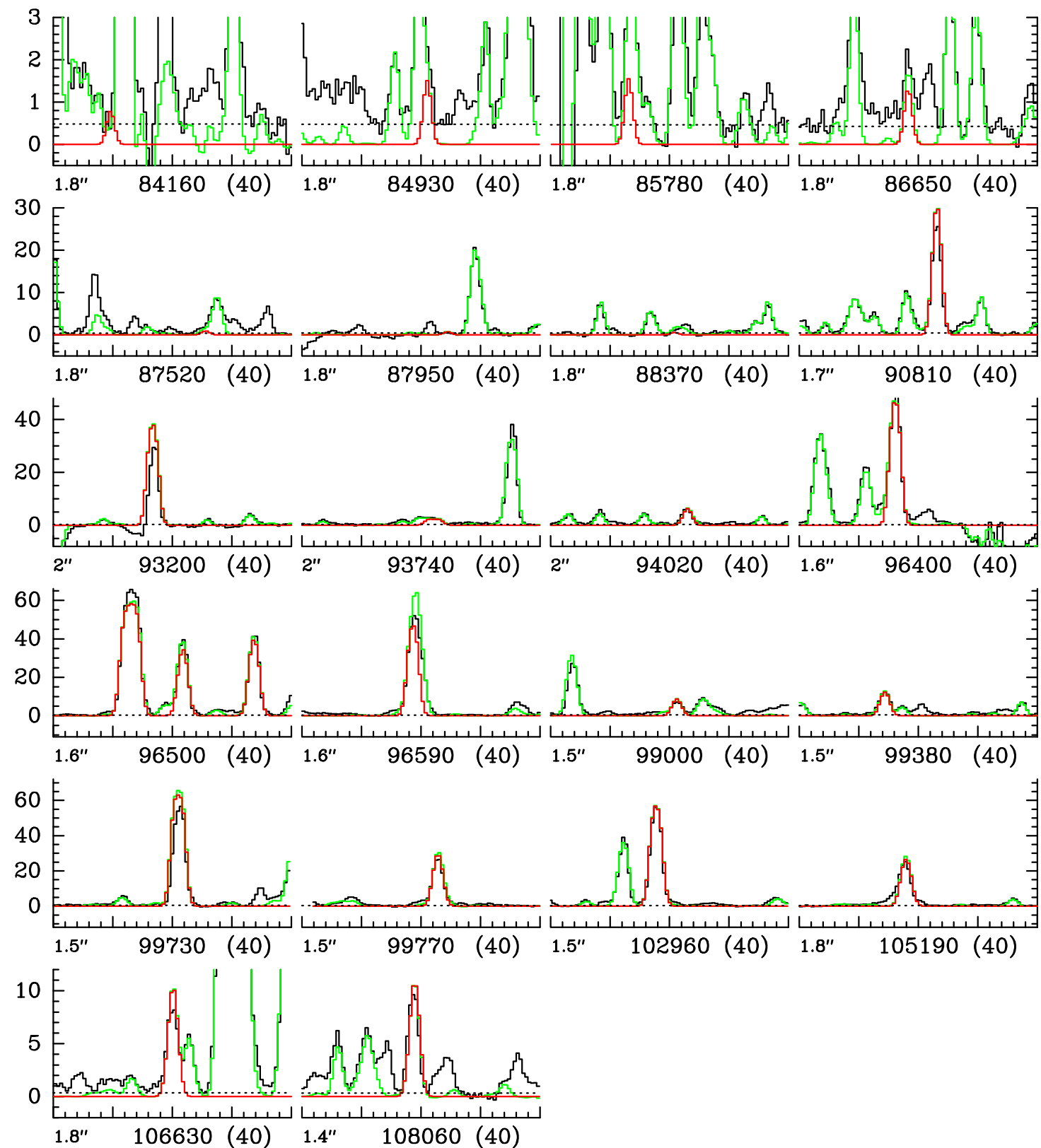

Fig. A.2. Same as Fig. A.1 for $\mathrm{CH}_{3} \mathrm{OH}, v_{\mathrm{t}}=1$.
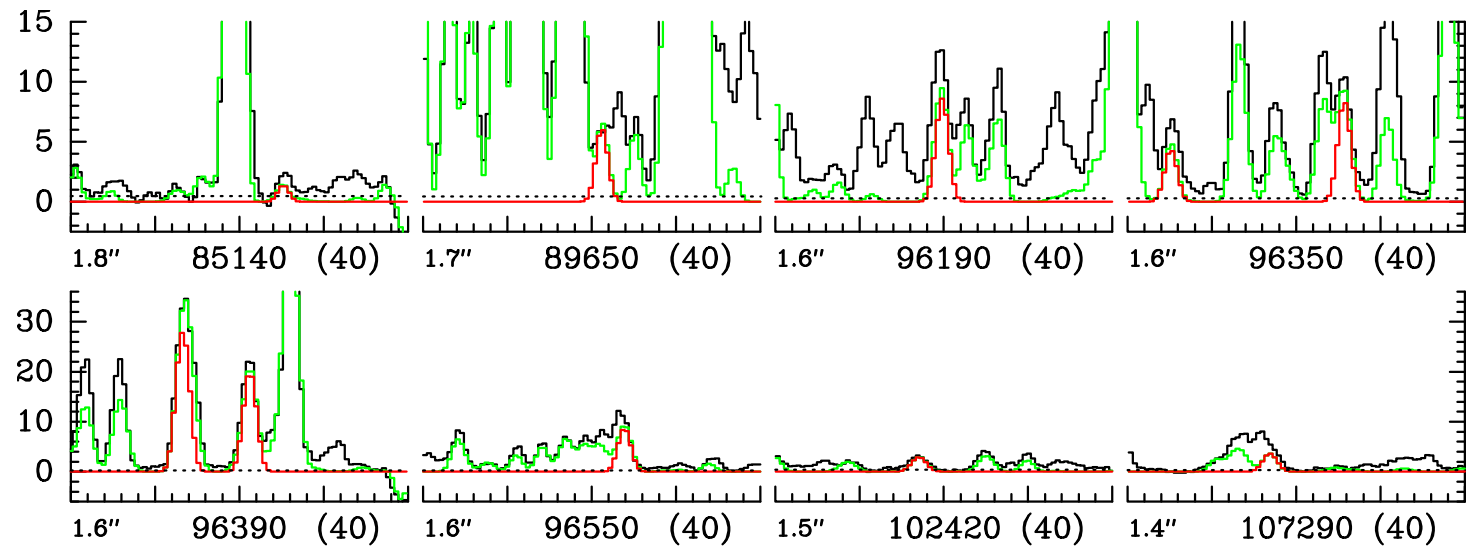

Fig. A.3. Same as Fig. A.1 for $\mathrm{CH}_{3} \mathrm{OH}, v_{\mathrm{t}}=2$. 
A\&A 587, A92 (2016)

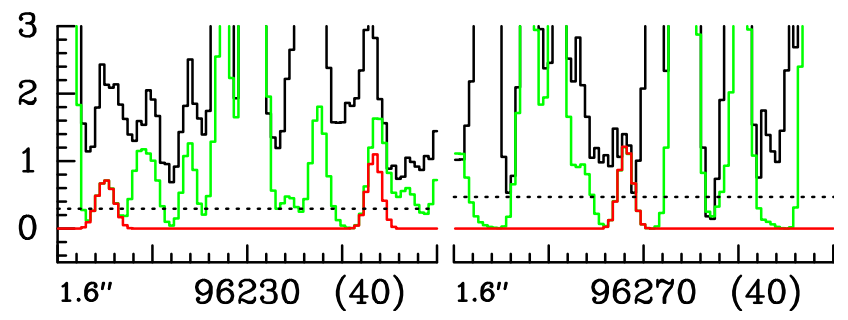

Fig. A.4. Same as Fig. A.1 for $\mathrm{CH}_{3} \mathrm{OH}, v_{\mathrm{t}}=3$. 
H. S. P. Müller et al.: Alkanethiols and alkanols in Sgr B2(N2)
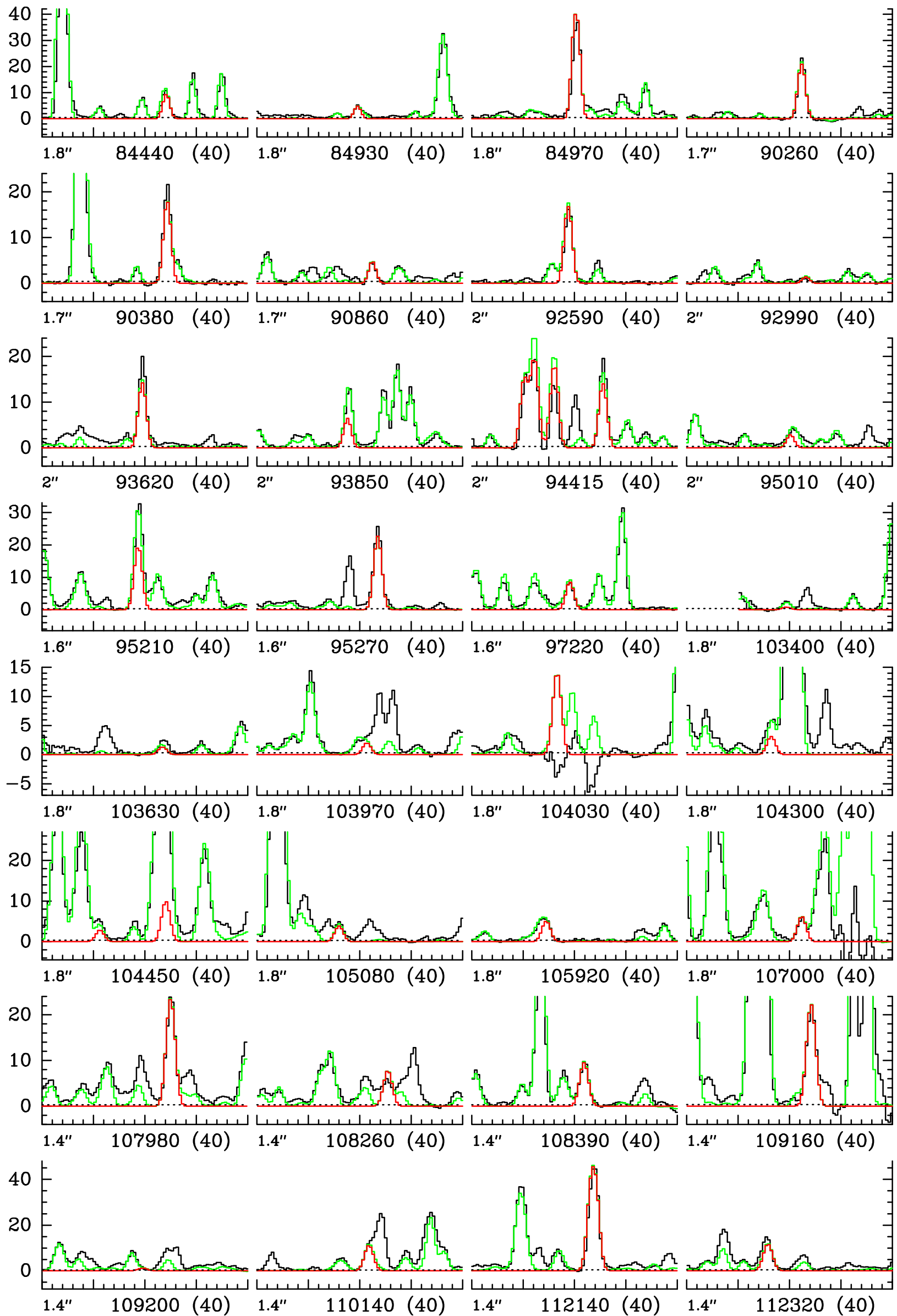

Fig. A.5. Same as Fig. A.1 for ${ }^{13} \mathrm{CH}_{3} \mathrm{OH}, v=0$. 


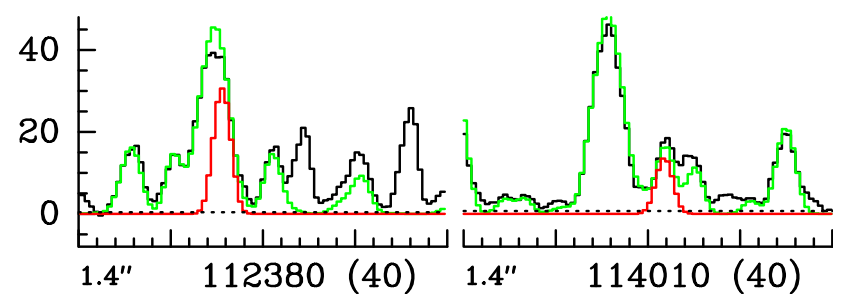

Fig. A.5. continued.

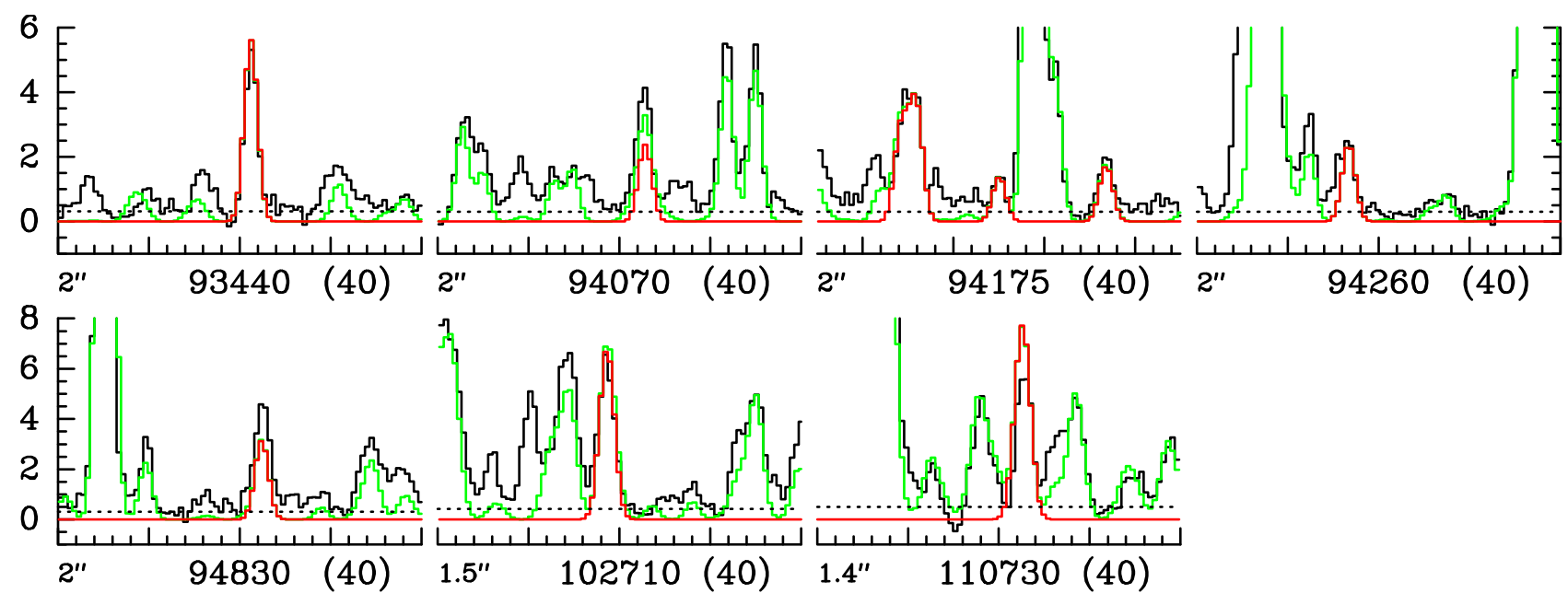

Fig. A.6. Same as Fig. A.1 for ${ }^{13} \mathrm{CH}_{3} \mathrm{OH}, v_{\mathrm{t}}=1$. 
H. S. P. Müller et al.: Alkanethiols and alkanols in Sgr B2(N2)
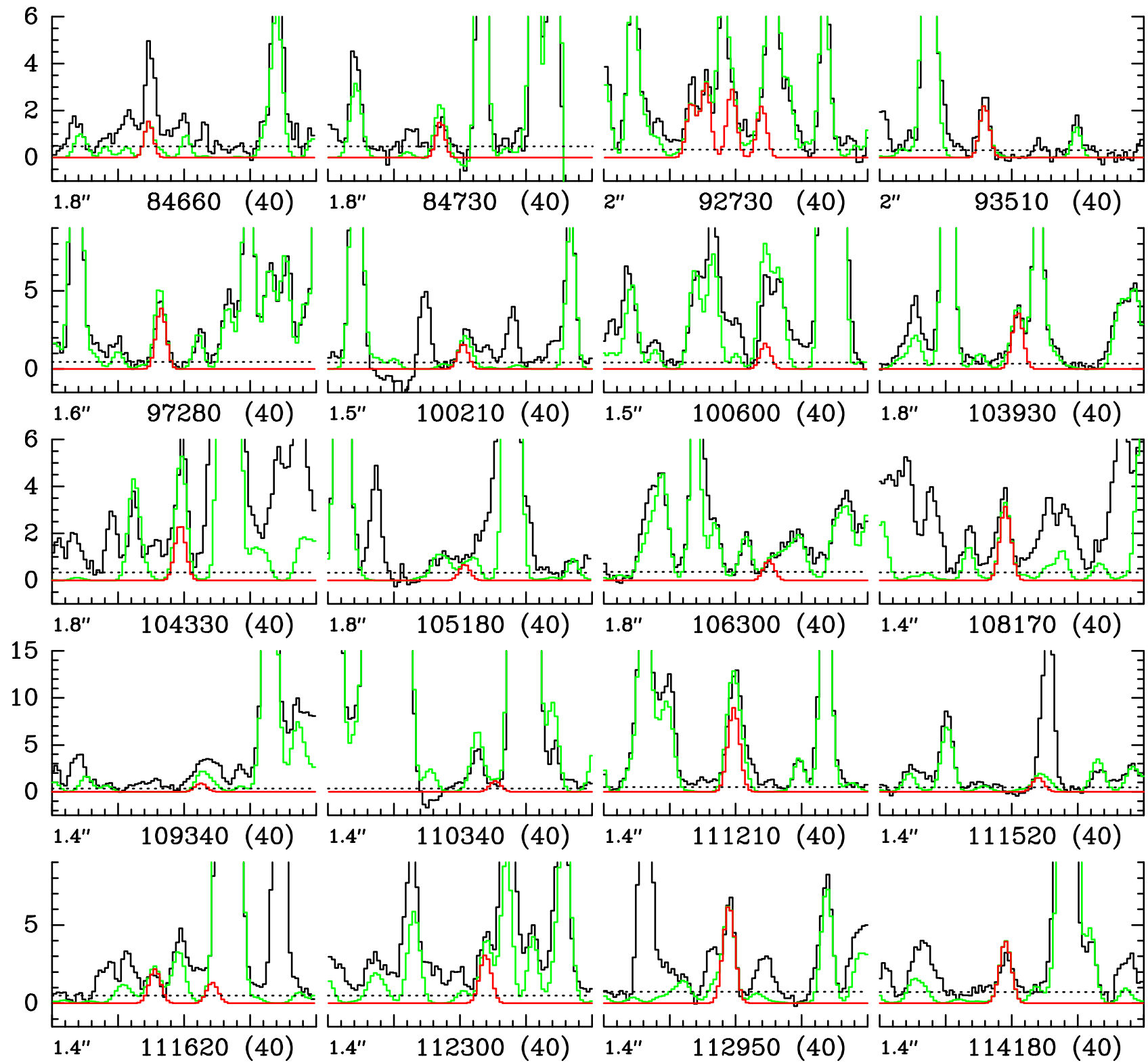

Fig. A.7. Same as Fig. A.1 for $\mathrm{CH}_{3}^{18} \mathrm{OH}, v=0$.

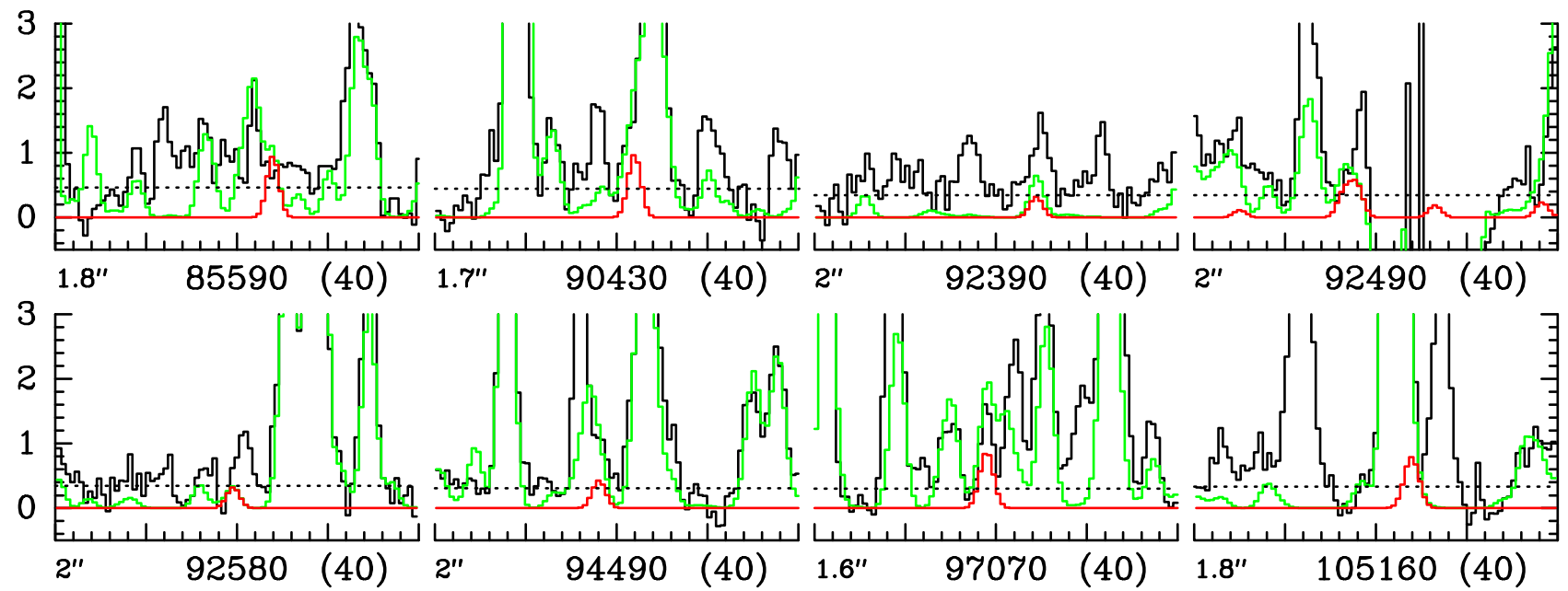

Fig. A.8. Same as Fig. A.1 for $\mathrm{CH}_{3}^{18} \mathrm{OH}, v_{\mathrm{t}}=1$. 
A\&A 587, A92 (2016)
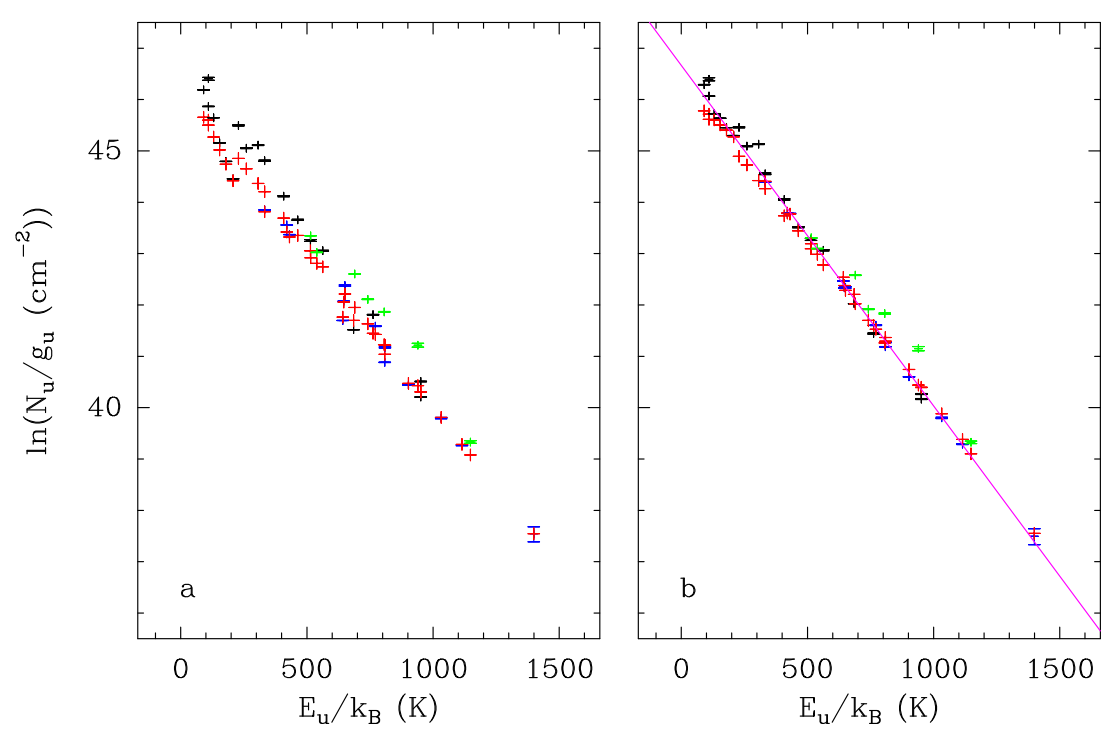

Fig. A.9. Population diagram of $\mathrm{CH}_{3} \mathrm{OH}, v=0, v_{\mathrm{t}}=1$, and $v_{\mathrm{t}}=2$ toward $\mathrm{Sgr} \mathrm{B} 2(\mathrm{~N} 2)$. Only the lines that are clearly detected, do not suffer too much from contamination from other species, and have an opacity below 2 are displayed. The observed data points are shown in black while the synthetic populations are shown in red. No correction is applied in panel a). In panel b), the optical depth correction has been applied to both the observed and synthetic populations and the contamination from all other species included in the full model has been removed from the observed data points. The straight line is a linear fit to the observed populations (in linear-logarithmic space). The rotational temperature derived in this way is reported in Table 6.
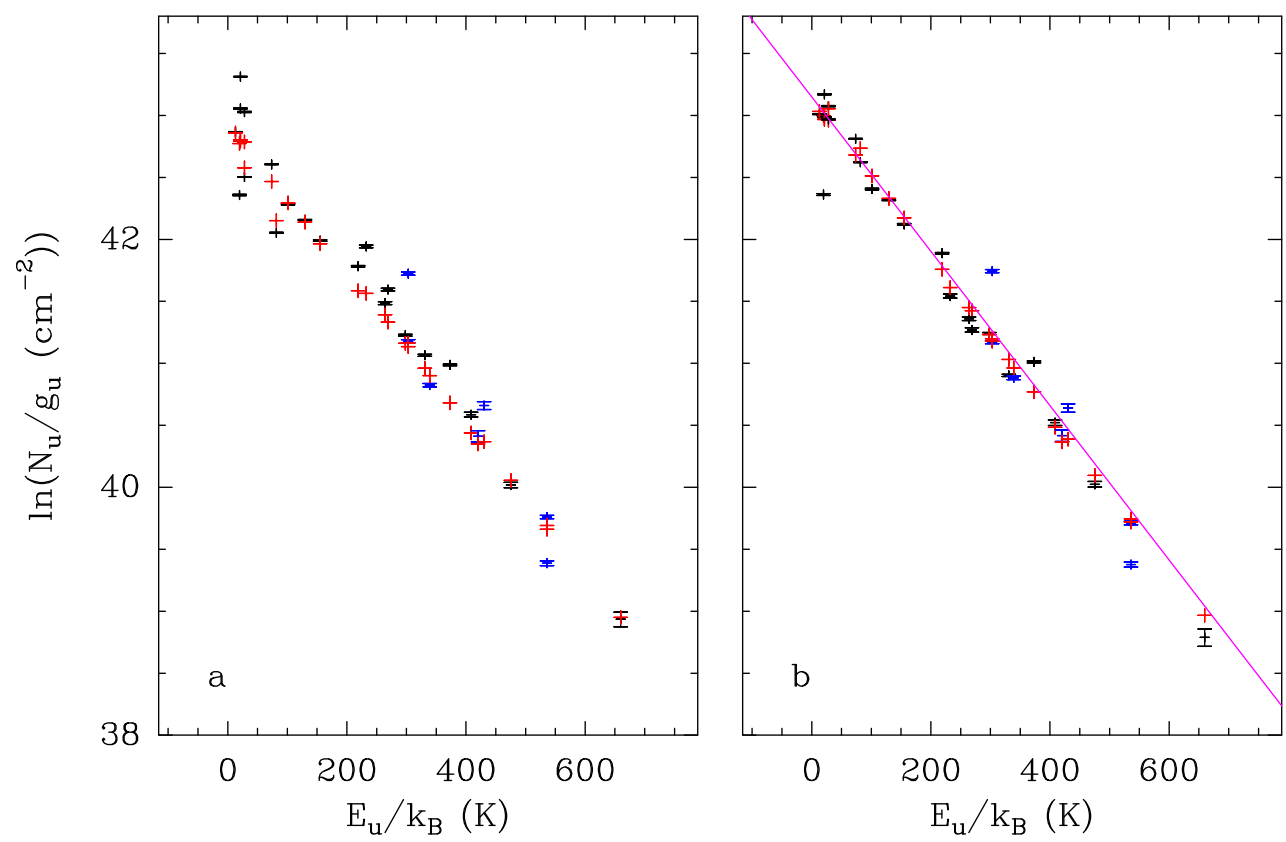

Fig. A.10. Same as Fig. A.9 for ${ }^{13} \mathrm{CH}_{3} \mathrm{OH}, v=0$ and $v_{\mathrm{t}}=1$. 
H. S. P. Müller et al.: Alkanethiols and alkanols in Sgr B2(N2)
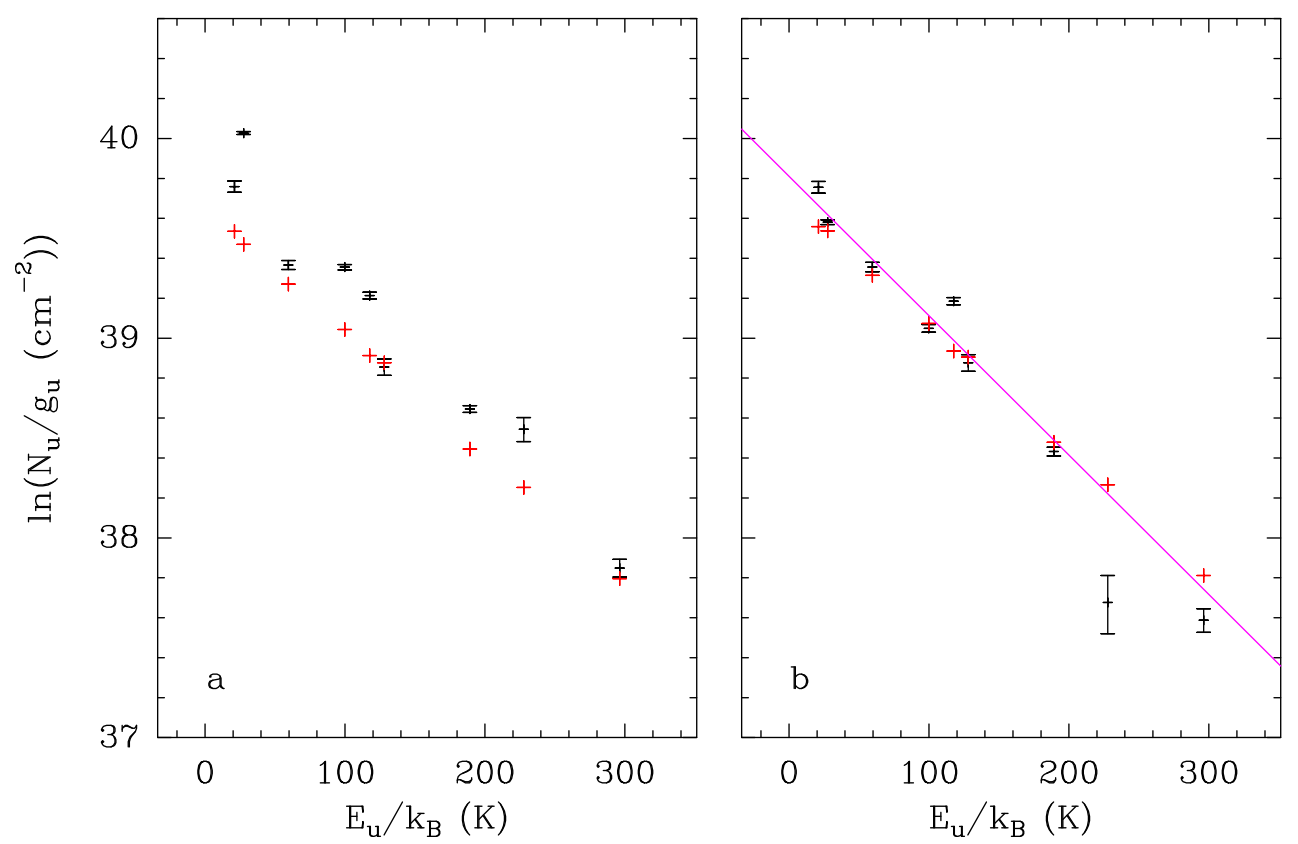

Fig. A.11. Same as Fig. A. 9 for $\mathrm{CH}_{3}^{18} \mathrm{OH}, v=0$. 
A\&A 587, A92 (2016)
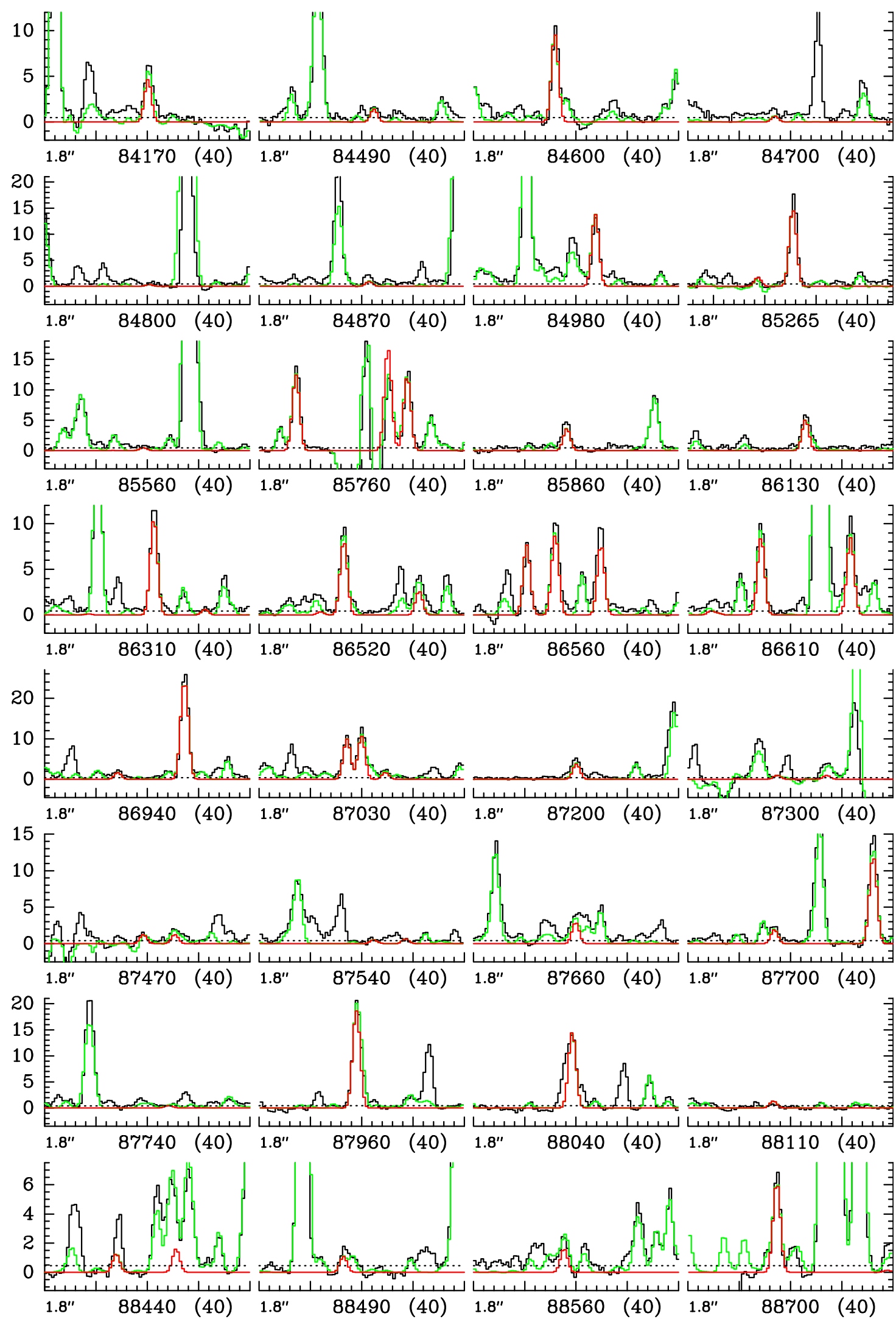

Fig. A.12. Same as Fig. A. 1 for $\mathrm{C}_{2} \mathrm{H}_{5} \mathrm{OH}, v=0$. 
H. S. P. Müller et al.: Alkanethiols and alkanols in Sgr B2(N2)
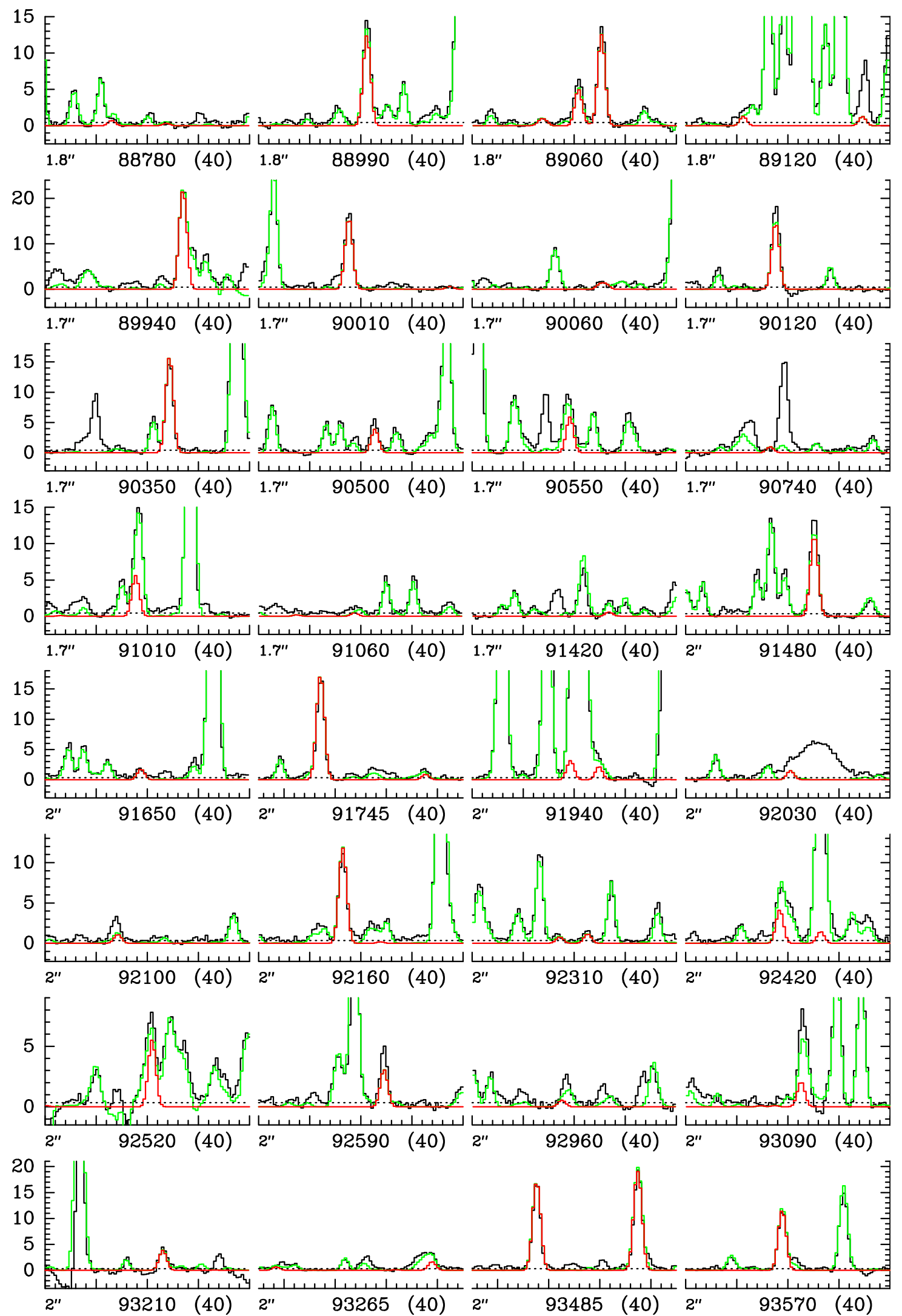

Fig. A.12. continued. 
A\&A 587, A92 (2016)
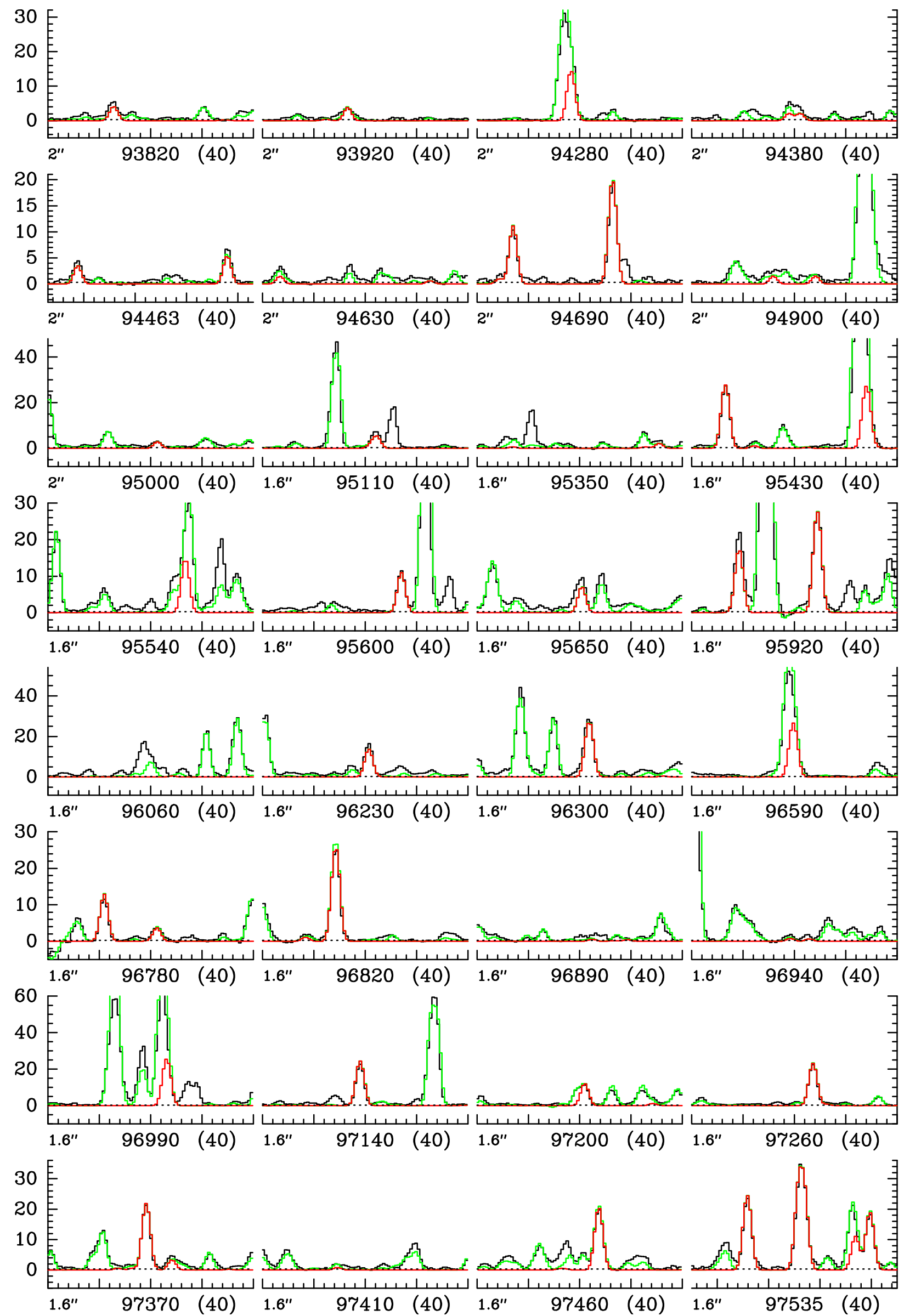

Fig. A.12. continued. 
H. S. P. Müller et al.: Alkanethiols and alkanols in Sgr B2(N2)
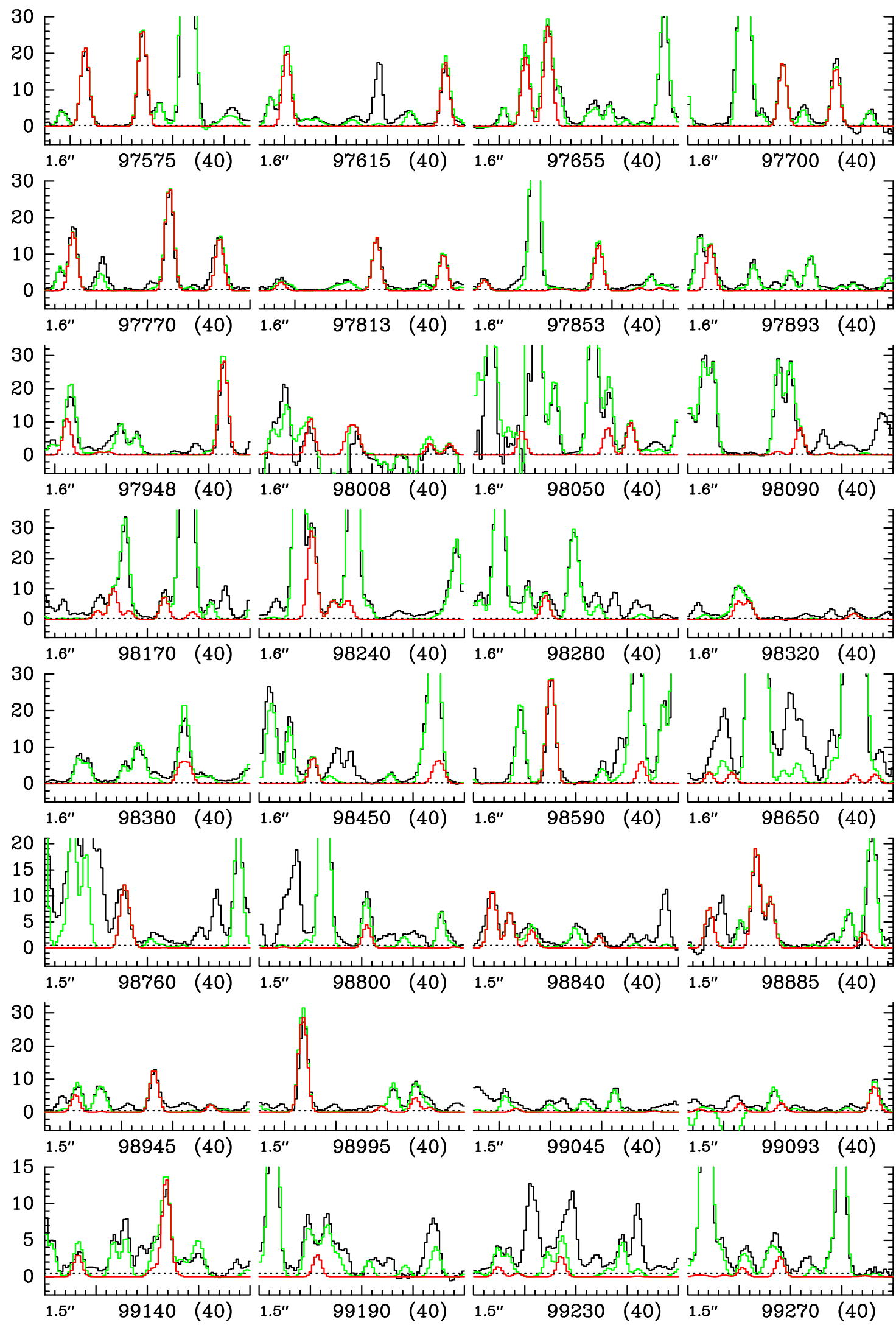

Fig. A.12. continued. 

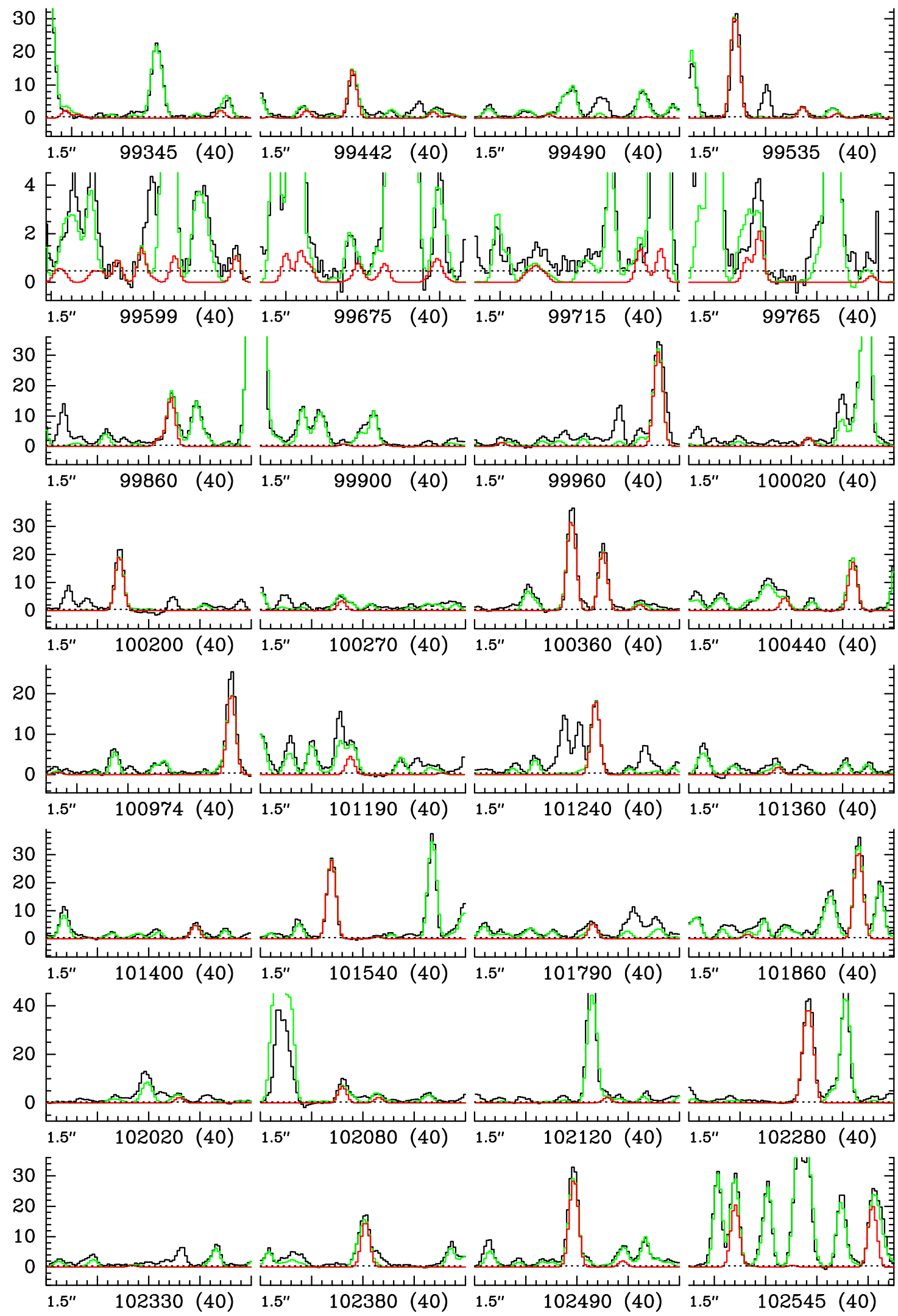

Fig. A.12. continued. 
H. S. P. Müller et al.: Alkanethiols and alkanols in Sgr B2(N2)
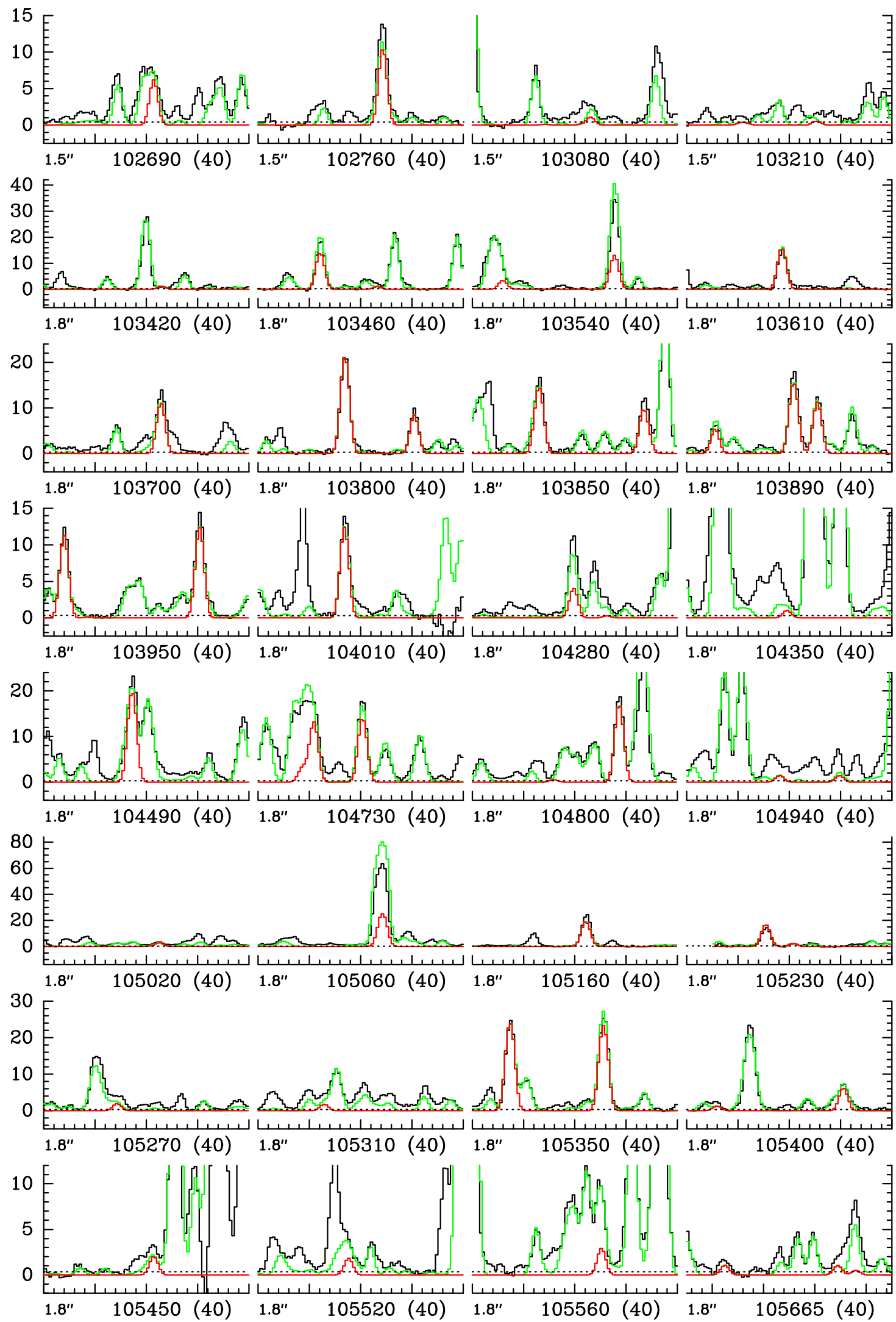

Fig. A.12. continued. 
A\&A 587, A92 (2016)
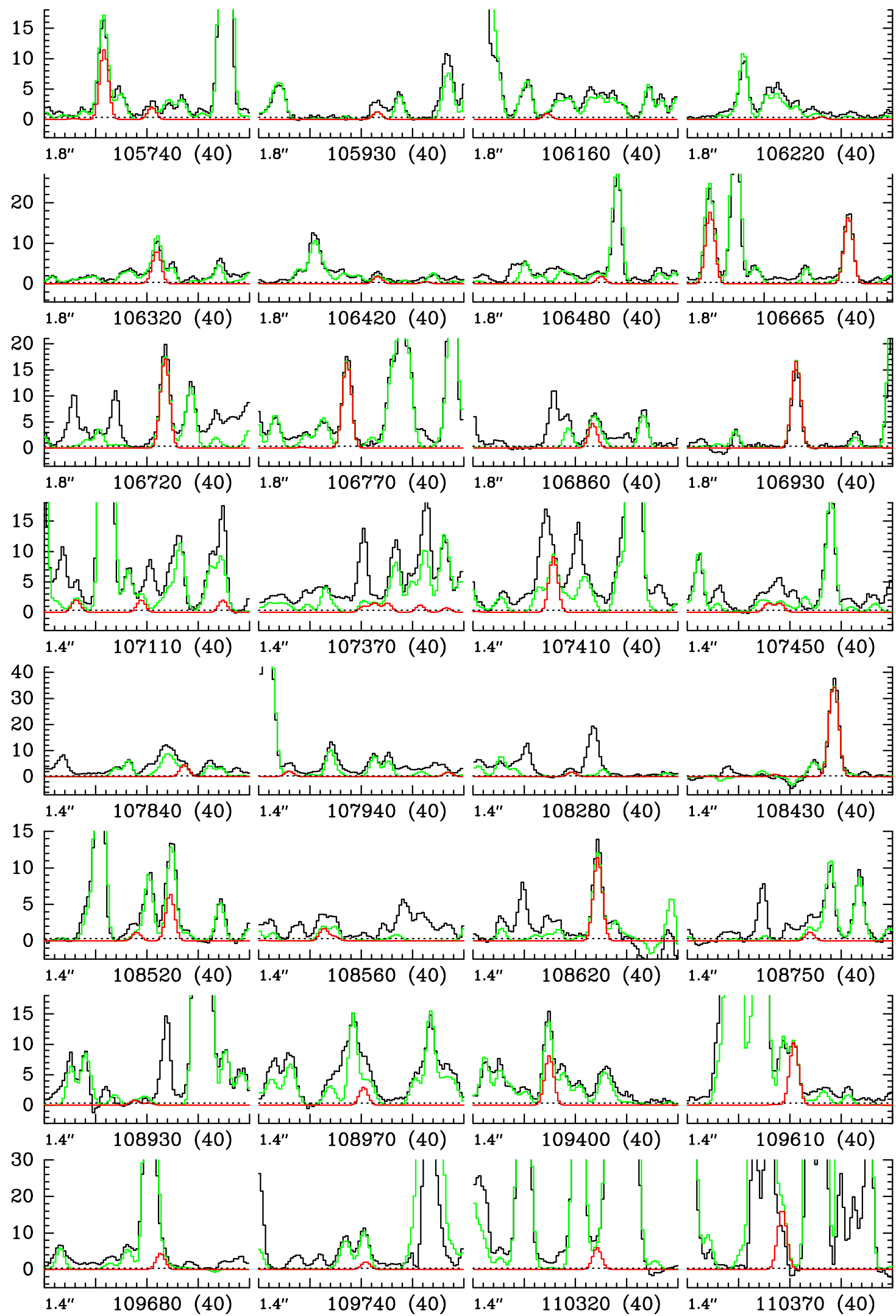

Fig. A.12. continued. 
H. S. P. Müller et al.: Alkanethiols and alkanols in Sgr B2(N2)
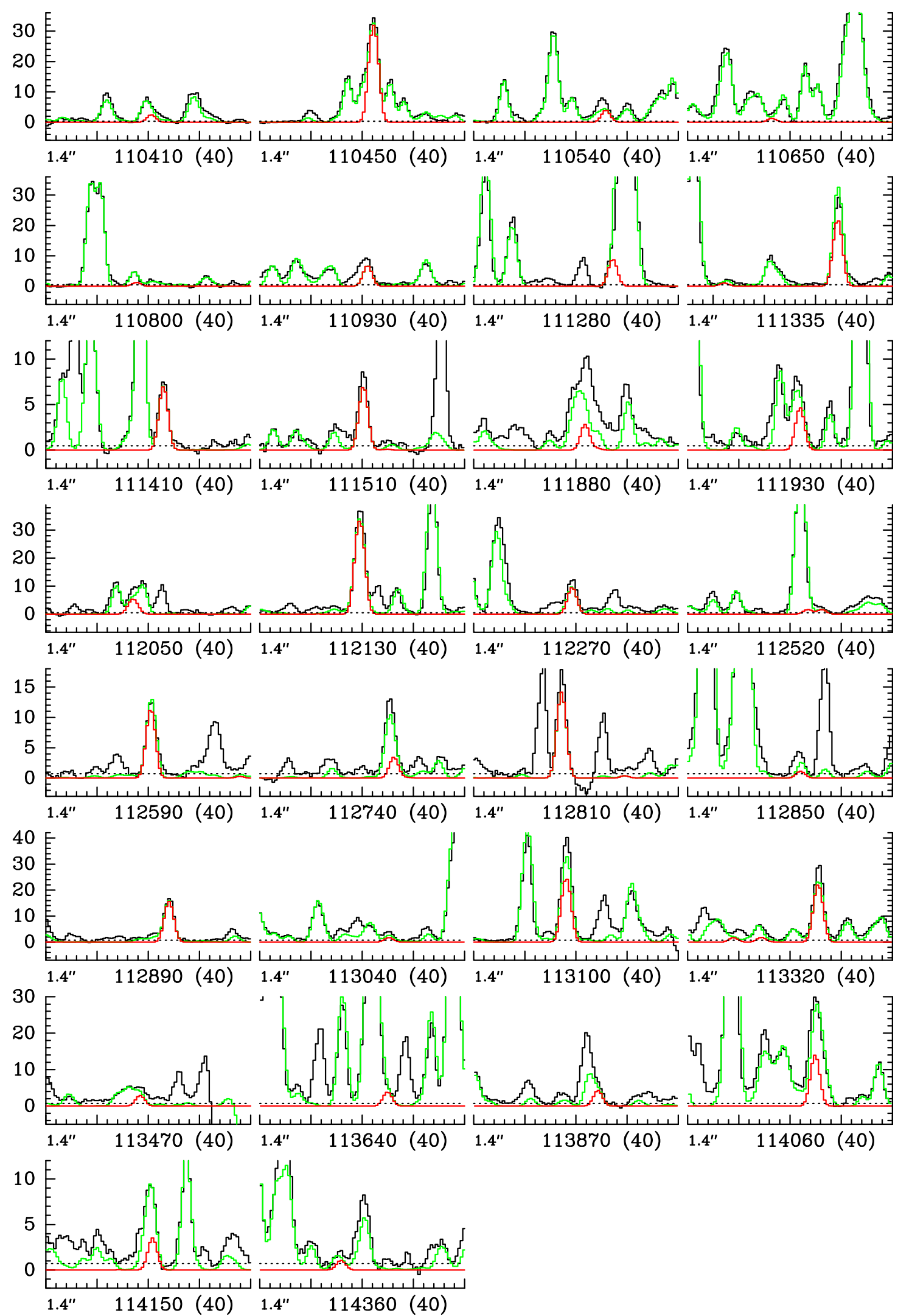

Fig. A.12. continued. 
A\&A 587, A92 (2016)
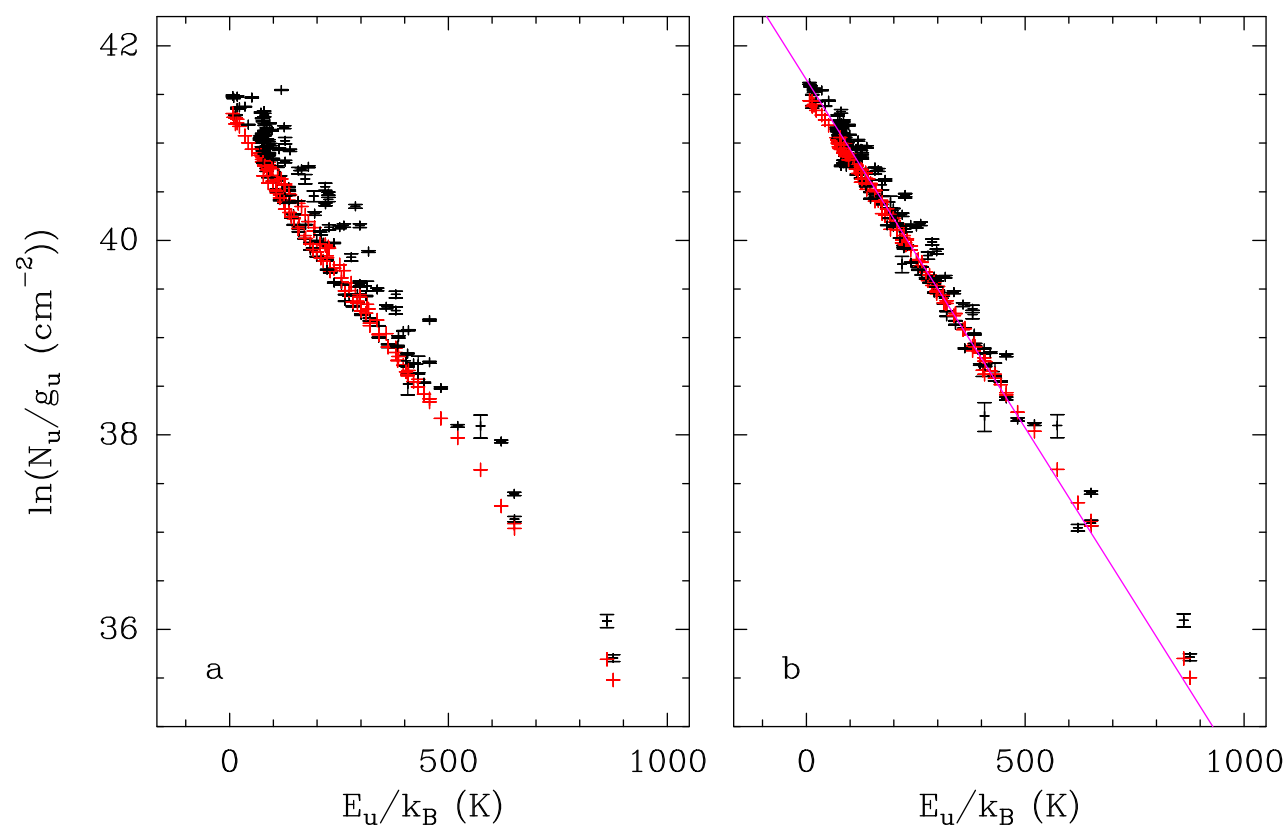

Fig. A.13. Same as Fig. A. 9 for $\mathrm{C}_{2} \mathrm{H}_{5} \mathrm{OH}, v=0$.
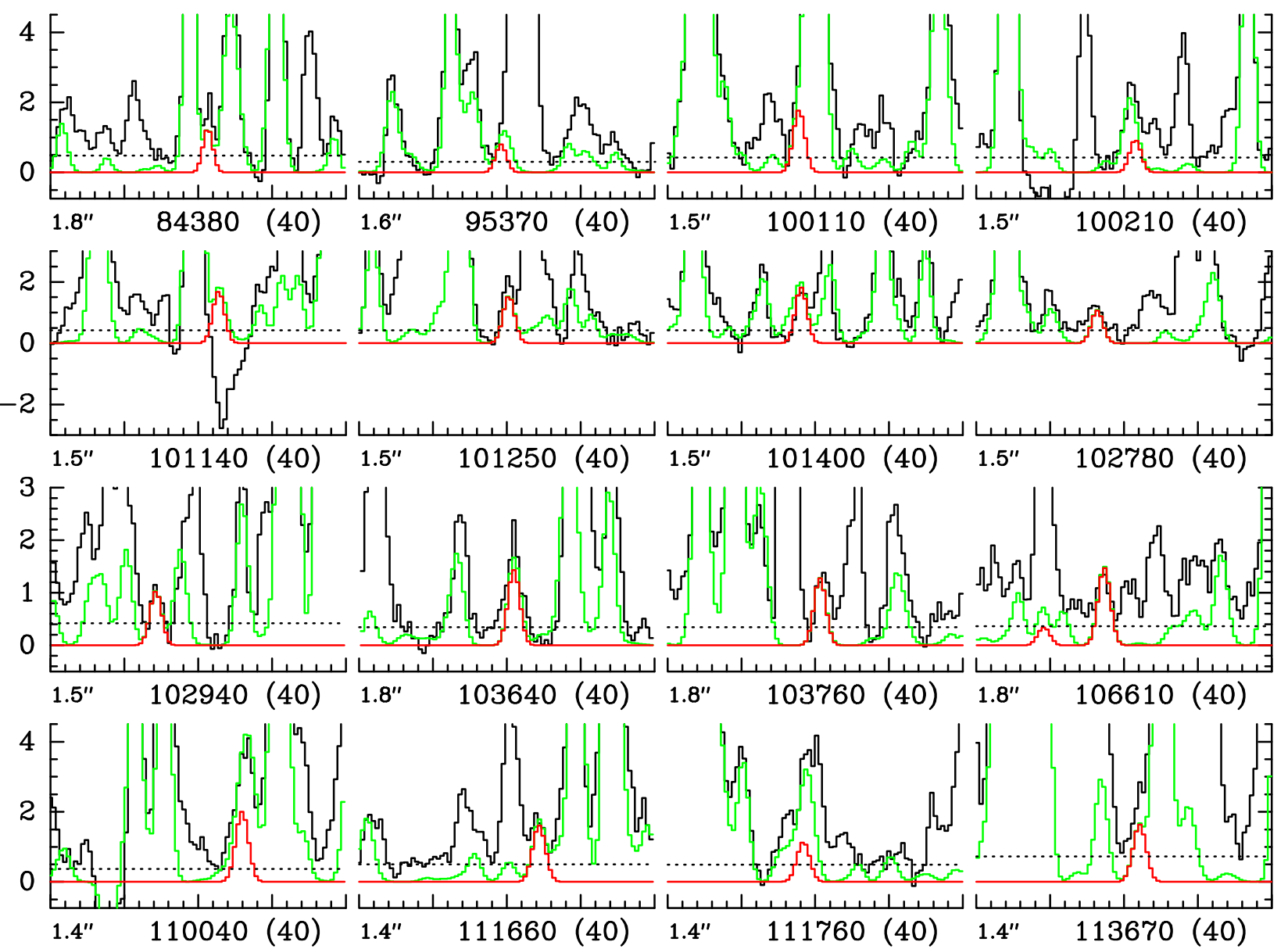

Fig. A.14. Same as Fig. A. 1 for ${ }^{13} \mathrm{CH}_{3} \mathrm{CH}_{2} \mathrm{OH}, v=0$. 
H. S. P. Müller et al.: Alkanethiols and alkanols in Sgr B2(N2)
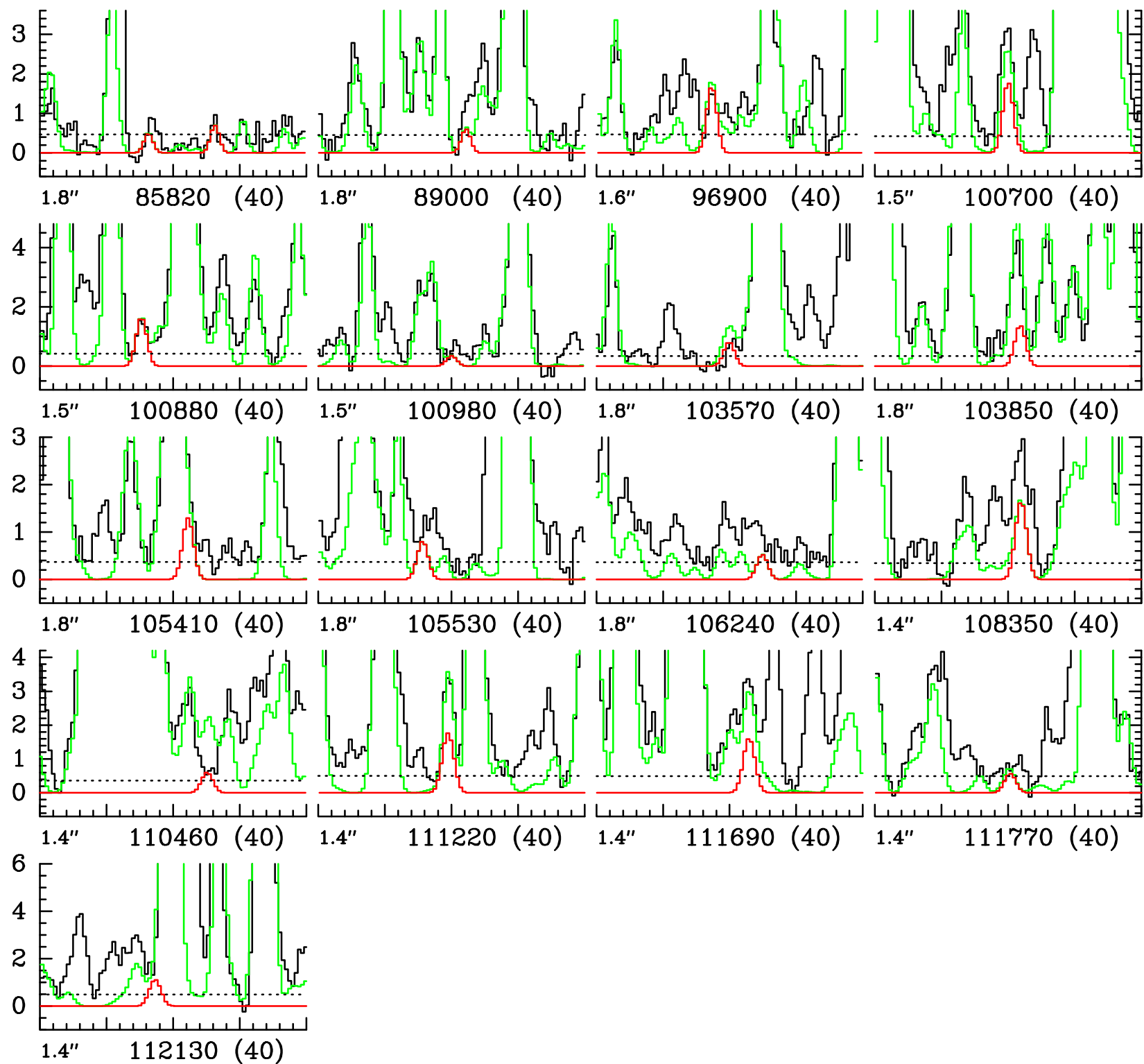

Fig. A.15. Same as Fig. A. 1 for $\mathrm{CH}_{3}^{13} \mathrm{CH}_{2} \mathrm{OH}, v=0$. 

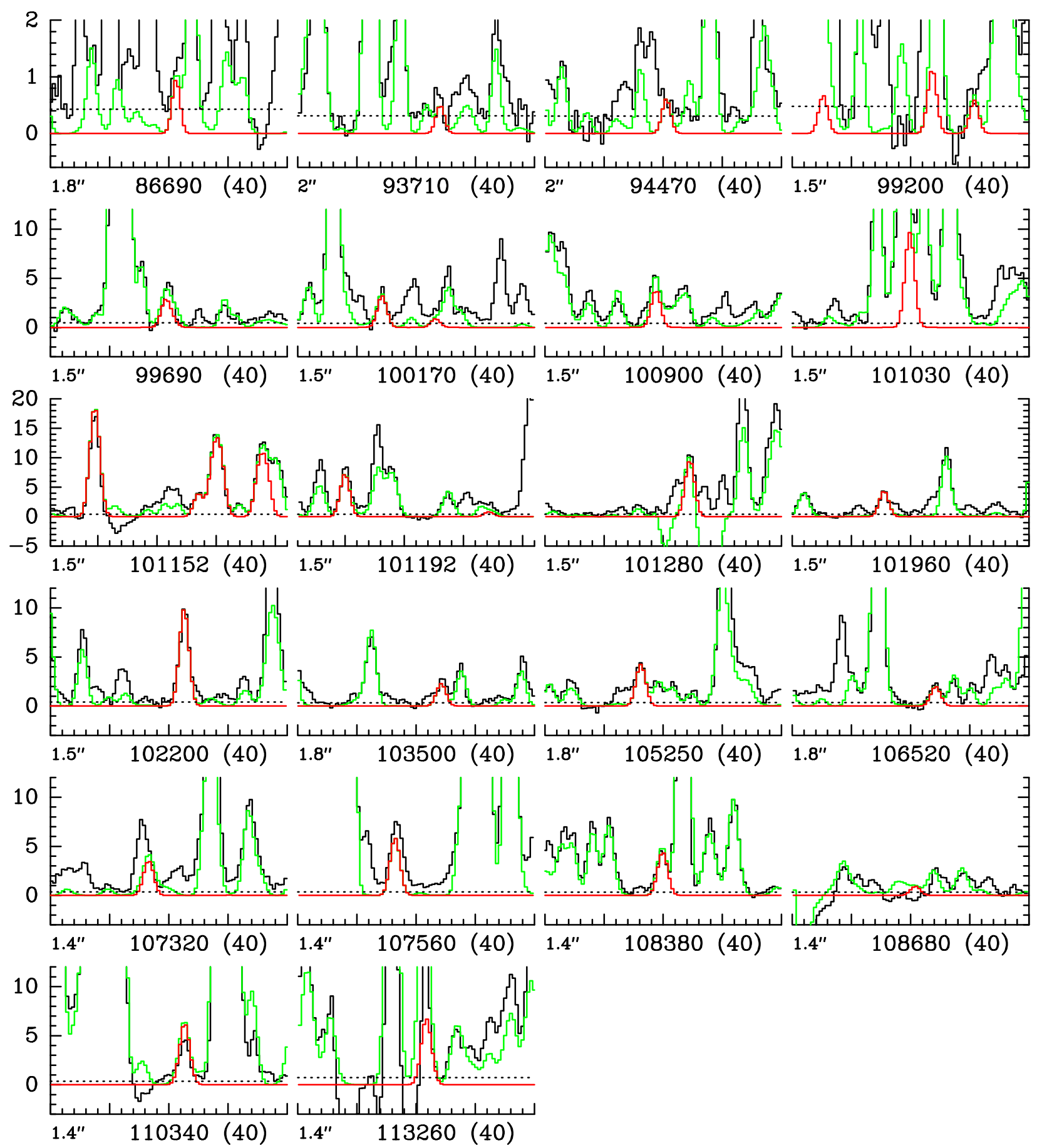

Fig. A.16. Same as Fig. A. 1 for $\mathrm{CH}_{3} \mathrm{SH}, v=0$. 
H. S. P. Müller et al.: Alkanethiols and alkanols in Sgr B2(N2)

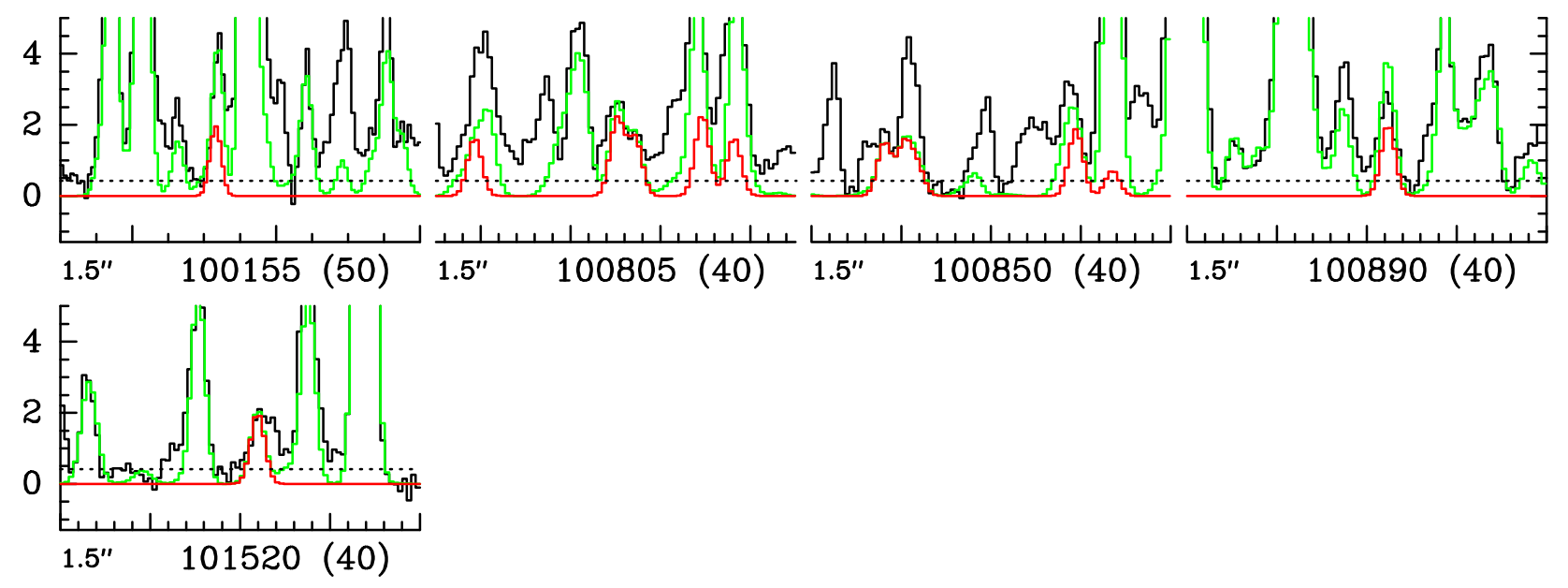

Fig. A.17. Same as Fig. A. 1 for $\mathrm{CH}_{3} \mathrm{SH}, v_{\mathrm{t}}=1$.
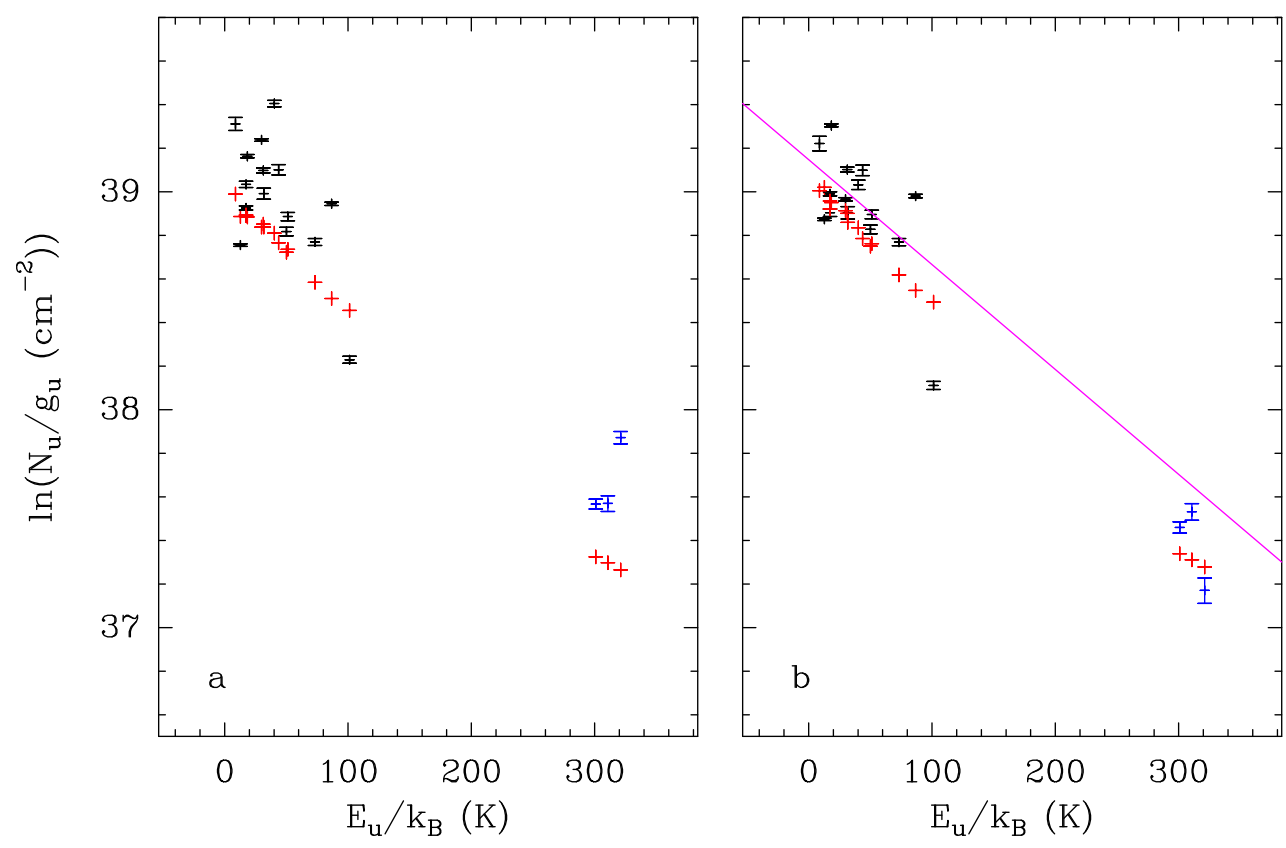

Fig. A.18. Same as Fig. A. 9 for $\mathrm{CH}_{3} \mathrm{SH}, v=0$ and $v_{\mathrm{t}}=1$. 
A\&A 587, A92 (2016)
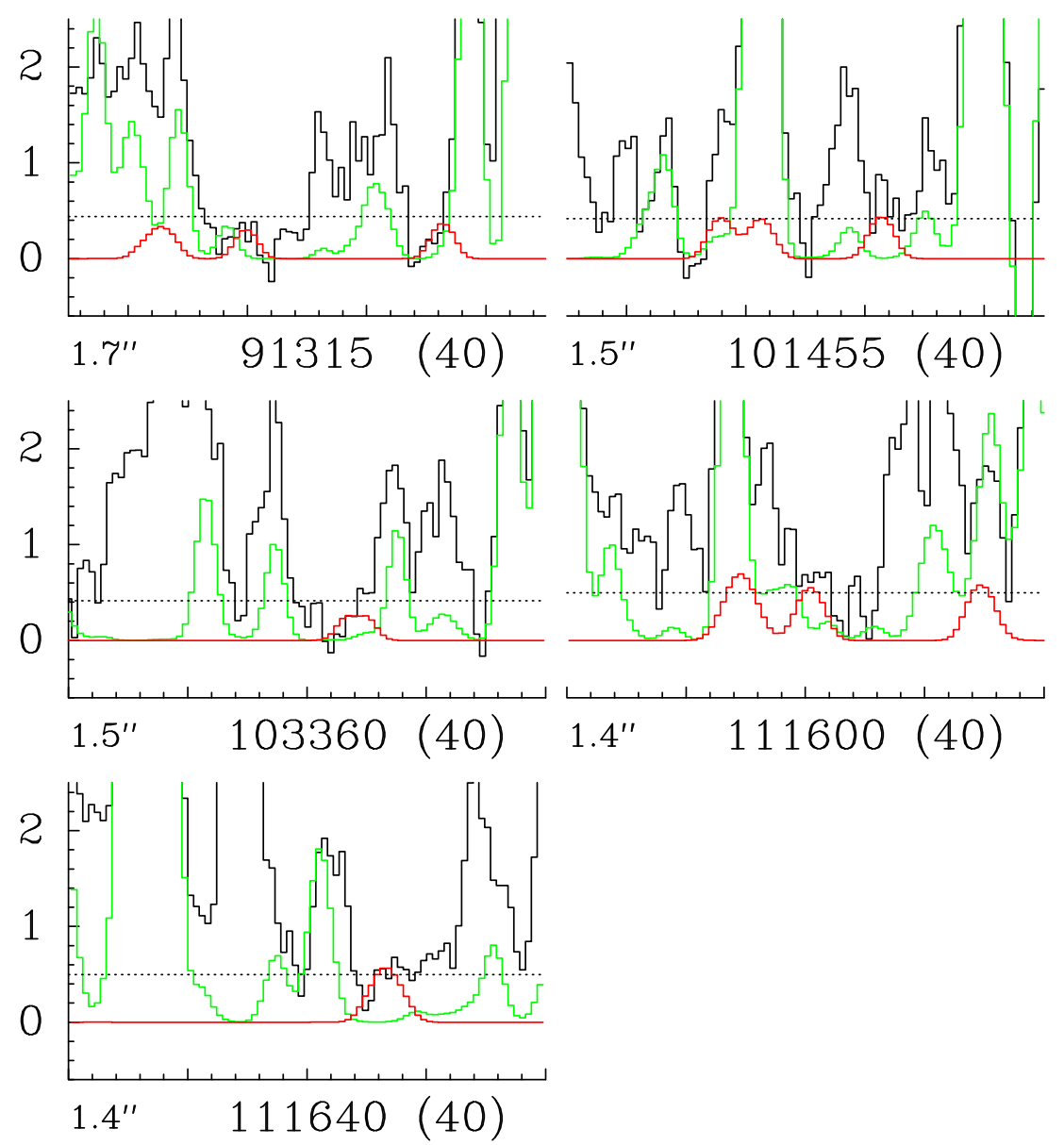

Fig. A.19. Same as Fig. A.1 for gauche- $\mathrm{C}_{2} \mathrm{H}_{5} \mathrm{SH}, v=0$, but here, the green synthetic spectrum does not contain the contribution of $g a u c h e-\mathrm{C}_{2} \mathrm{H}_{5} \mathrm{SH}$ that is shown in red. 